Universidade de São Paulo

Instituto de Física de São Carlos

Departamento de Física e Informática

\title{
Tunelamento Assistido em Metais
}

USP/IFSC/SBI

Luis Roberto Ramos

Dissertação apresentada ao Instituto de Física de São Carlos, Universidade de São Paulo, para obtenção do Título de Mestre em Ciências: Física Básica

Orientador: Prof. Dr. Valter Luiz Líbero

São Carlos

1998 


Ramos, Luis Roberto
Tunelamento Assistido em Metais/Luis Roberto Ramos.-- São Carlos,
1998.
$70 \mathrm{p}$.
Dissertação (Mestrado)--Instituto de Física de São Carlos, 1998.
Orientador: Prof. Dr. Valter Luiz Líbero
1. Metais. 2. Modelo de Kondo de Tunelamento.
3. Propriedades Termodinâmicas
I. Título.
I. Título. 
MEMBROS DA COMISSÃO JULGADORA DA DISSERTAÇÃO DE MESTRADO DE LUIS ROBERTO RAMOS APRESENTADA AO INSTITUTO DE FÍSICA DE SÃO CARLOS, DA UNIVERSIDADE DE SÃO PAULO, EM 06 DE ABRIL DE 1998.

COMISSÃO JULGADORA

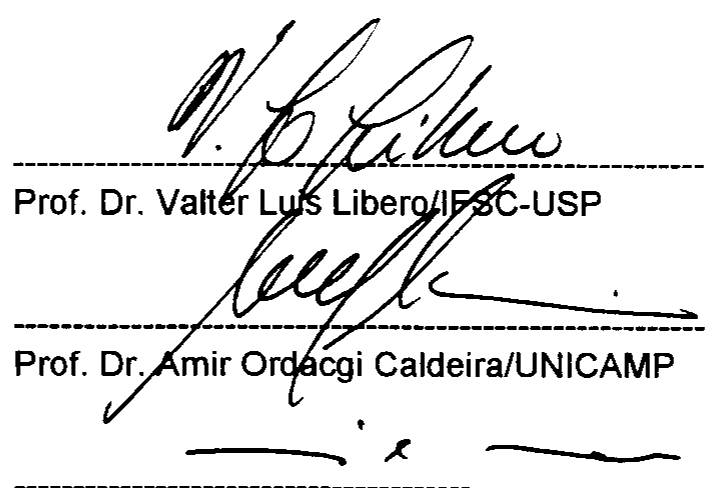

Prof. Dr. Miled Hassan Youssef Moussa/UFSCar 
Este trabalho teve o apoio financeiro da FAPESP.

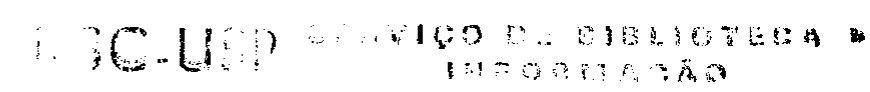




\section{Agradecimentos}

Não gostaria de citar nomes nesse agradecimento, mas sim agradecer a todos que de uma maneira direta ou não, contribuiram para que esse trabalho fosse realizado. Entre eles estão todos os meus companheiros de grupo, professores, colegas de sala e tanto outros.

Em especial, agradeço ao meu orientador, Prof. Valter Luiz Líbero, pela sua dedicação que foi de fundamental importância para mim. A sua conduta e apoio sempre me serviram de estímulo para completar esse trabalho.

Por fim, agradeço a força que toda minha família me deu (e continua dando) para que eu pudesse prosseguir os estudos.

Muito obrigado a todos. 


\section{Índice}

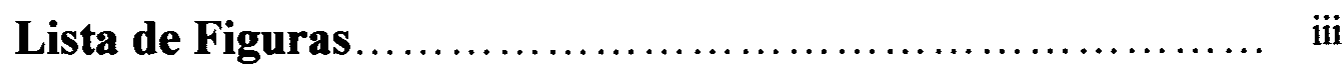

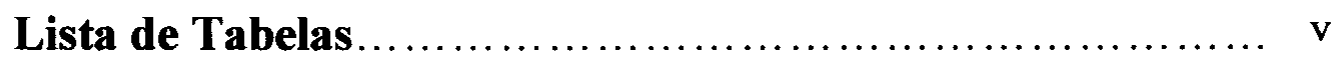

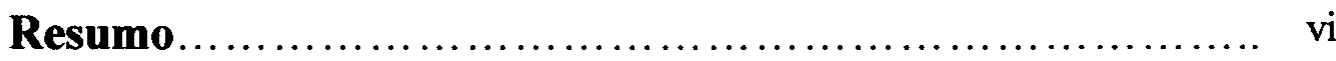

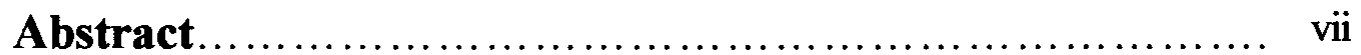

1 - Introdução 1

1.1 - Considerações Gerais.................................................. 1

1.2 - O Modelo....................................................... 3

1.3 - Grupo de Renormalização Numérico................................ 5

1.4 - Resumo dos resultados........................................ 6

1.5 - Organização Geral do Trabalho....................................... 7

2 - $O$ modelo de Kondo de Tunelamento 9

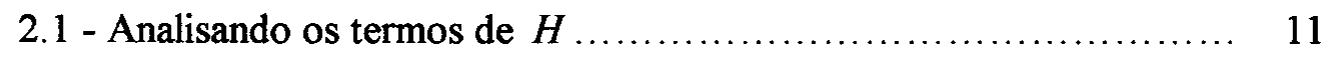

2.1.1 - Hamiltoniano da Banda de Condução........................... 11

2.1.2 - Hamiltoniano de Tunelamento Direto ......................... 11

2.1.3 - Hamiltoniano de Interação ................................. 12

2.2 - Hamiltoniano de Tunelamento Assistido............................. 15

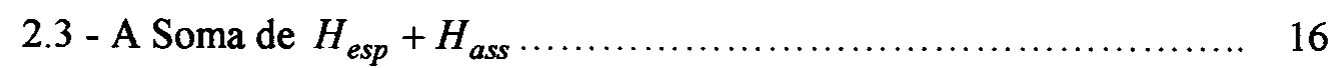

2.4 - Hamiltoniano Completo............................................. 18

3 - Grupo de Renormalização Numérico 19

3.1 - Adaptação do Hamiltoniano de Dois Centros ao GRN............... 19 
3.1.1 - Transformação do Operador de Campo

3.1.2 - Transformação dos Termos de Espalhamento e Tunelamento Assistido

3.1.3 - Transformação da Banda de Condução.

3.1.4 - Hamiltoniano Completo. 26

3.2 - Discretização Logarítmica. 27

3.3 - Truncamento. 32

3.4 - As Simetrias do Problema 36

3.5 - Diagonalização Iterativa. 37

3.6 - A Transformação do GRN e os Pontos Fixos. 39

3.6.1 - Banda Livre.

4.1 - Calor Específico 45

4.2 - Calor Específico sem Tunelamento Assistido 47

4.3 - Tunelamento Assistido.

4.4 - O Problema Kondo de Impureza Magnética e o Tunelamento Assistido

4.5 - Situação Kondo incluindo $\Delta \neq 0$

4.6 - Situação Kondo incluindo $G_{1} \neq 0$

4.7 - Comportamento Geral do Calor Específico com $G_{a}$

4.8 - Cálculo da Taxa Efetiva de Tunelamento 


\section{Lista de Figuras}

1.1 a) Esquematização de um sistema de dois níveis em um metal amorfo; b) potencial efetivo que descreve seu movimento

1.2 A curva universal do calor específico do modelo de Kondo é representada nesta figura pelos pontos vermelhos.

1.3 Comportamento de várias curvas de calor específico quando variamos o parâmetro de tunelamento assistido.

2.1 Representação esquemática de um íon num metal tunelando entre dois sítios equivalentes

2.2 Representação esquemática do problema de dois níveis localizados em um metal.

2.3 Representação esquemática da banda de condução do metal. A banda é isotrópica e simétrica em ralação ao nível de Fermi $\left(\varepsilon_{F}\right)$ e com largura $2 D$

2.4 Espalhamento dos elétrons de condução pelo íon no processo de tunelamento assistido.

3.1 Banda de condução discretizada logaritmicamente

3.2 Curvas de calor específico mostrando o efeito do procedimento intercalado

3.3 Banda de condução representada por alguns de seus níveis (centrada no nível de Fermi e com largura 2D).

3.4 Diagrama de fluxo de energia do modelo de Kondo de Tunelamemento, mostrando a evolução das energias mais baixas para $\mathrm{N}$ ímpar.

4.1 Curvas de calor específico com valores diferentes de $G$ e $\Delta$ e com $G_{a}=0$ 
4.2 Representação esquemática do alargamento das linhas de energia dos orbitais ligante e antiligante, quando o sistema de dois níveis interage com a BC

4.3 As curvas acima mostram que $G_{a}$ pode ter o mesmo efeito de $\Delta$, como pode ser obeservado pelas curvas azul e preta $\left(G_{a} \neq 0\right.$ e $\left.\Delta=0\right)$ e pela curva com pontos vermelha $\left(G_{a}=0\right.$ e $\left.\Delta \neq 0\right)$

4.4 Representação do modelo de Kondo para uma impureza magética com spin $\vec{\tau}$ em um metal.

4.5 Níveis de energia do estado singleto (estado fundamental) e dos estados tripletos

4.6 A curva vermelha nessa figura representa a curva universal de calor específico para o problema Kondo (extraídos da

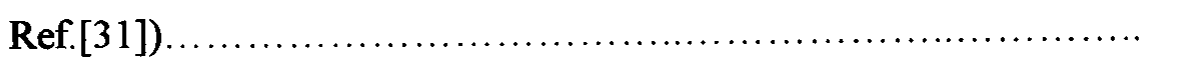

4.7 Representação esquemática da mistura dos níveis que possuem $S_{z}=0$ quando fazemos $\Delta \neq 0$

4.8 Curvas de calor específico; a primeira (curva azul) está no regime Kondo e a segunda (curva vermelha) e terceira (curva verde) foram obtidas acrescentado-se $\Delta \neq 0$

4.9 Curvas de calor específico: uma no regime Kondo (curva azul) e a outra com $G_{1} \neq 0$ (curva vermelha).

4.10 Várias curvas de calor específico, mostrando o comportamento geral do efeito do tunelamento assistido

4.11 As curvas acima mostram o comportamento de $\Delta^{*}$ em função do parâmetro de tunelamento assistido, $G_{a}$...

4.12 As curvas acima mostram que o comportamento das curvas da Fig. 4.11 coincidem quando normalizamos os valores de $G_{a}$ pelo seu respectivo $G_{a_{\min }}$ e os valores de $\Delta^{*}$ pelos valores de $\Delta^{\sim}$, sendo esta a taxa efetiva de tunelamento quando $G_{a}=0$, 


\section{Lista de Tabelas}

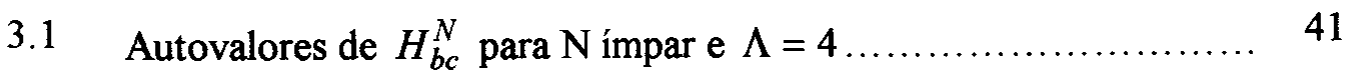

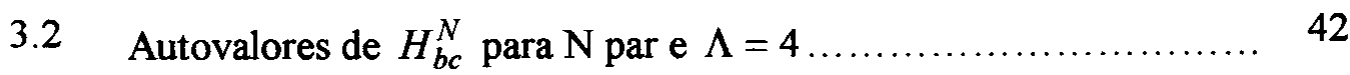




\section{Resumo}

Este trabalho mostra um modelo onde um íon sem spin tunela entre dois mínimos de potencial em um metal e interage eletrostaticamente com os elétrons de condução. Este modelo foi proposto por Kondo em 1976, sendo que ele não considerou a possibilidade do tunelamento ocorrer via espalhamento dos elétrons de condução. Este processo é conhecido como tunelamento assistido, e neste trabalho, nós o estamos levando em consideração. Para diagonalizar o Hamiltoniano que representa o modelo nós utilizamos o Grupo de Renormalização Numérico.

Estamos mostrando o calor específico como função da temperatura no caso onde não há tunelamento assistido e no caso onde ele está presente. Este trabalho mostra, também, que para uma escolha apropriada de parâmetros, este modelo é mapeado no famoso Hamiltoniano de Kondo para uma impureza magnética em metal.

Mostramos, ainda, o comportamento da taxa efetiva de tunelamento em função do parâmetro que representa o tunelamento assistido. Em especial, verifica-se que essa taxa pode, em alguns casos, ser maior que a taxa de tunelamento livre. 


\section{Abstract}

This work shows a model where a spinless ion tunnels between a double potencial well in a metal and interacts eletrostatically with the electrons of conduction band. This model was proposed by Kondo in 1976, but he did not consider the possibility of a tunneling caused by a scattering of conduction electron. This process is called assisted tunneling, and in this work, we take it into account. Numerical Renormalization Group is used to diagonalize the Hamiltonian representing the model.

We are showing here curves of specific heat as a function of temperature in the case where there is no assisted tunneling and in the case where it is present. This work also shows that for an appropriate choice of parameters this model maps in the famous Kondo Hamiltonian for a magnetic impurity in metal.

Finally, we are showing the behaviour of the effective tunneling rate as a function of the parameter that represent the assisted tunneling. In special, the results show that the rate may be, in some cases, larger that the bare tunneling rate.

vii

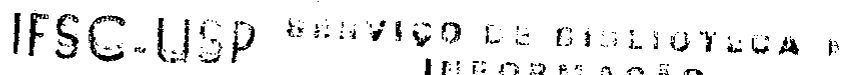
If i: 0 a 


\section{Capítulo 1}

\section{Introdução}

\section{1 - Considerações Gerais}

O problema de centros tunelantes (CT) tem atraído bastante atenção há muito tempo $[1,2,3,4]$. CT são formados por alguma partícula pesada (um átomo, um íon, etc.) movendo-se em um potencial efetivo através de mínimos locais. Se a barreira entre esses mínimos é suficientemente grande e os mínimos estão próximos o suficiente entre si, então a baixas temperaturas o "pulo" via ativação térmica é desprezível e o átomo pode se mover somente por tunelamento de um mínimo para outro [5]. A este tunelamento, que advém da superposição das funções de onda centradas nos mínimos locais, denominaremos nesta dissertação de tunelamento direto.

O caso mais simples de CT é justamente o de um íon movendo-se entre dois mínimos de potencial, sendo que em cada mínimo local temos associado uma autofunção e um nível de energia. Isto origina um sistema de dois níveis, o qual se torna mais interessante se estiver acoplado a um meio metálico. Isto é o que acontece, por exemplo, em metais amorfos como ligas de $\mathrm{Ni}_{75} \mathrm{P}_{25}[5,6]$.

São apontados dois procesos distintos nos quais os elétrons de condução podem interagir dinamicamente com o íon [4]:

(i) No primeiro processo os elétrons de condução tendem a formar uma blindagem ao redor do íon tunelante devido ao acoplamento eletrostático. Como conseqüência a taxa de tunelamento do íon diminui. 
(ii) Podemos ter também, além da blindagem, o chamado tunelamento assistido por elétrons de condução. Neste processo, o tunelamento ocorre através de um espalhamento dos elétrons de condução.

Tem sido apontado por alguns autores que esse processo é devido às flutuações da barreira de potencial causada pelas flutuações da densidade eletrônica local $[7,8]$, ou também, por transições virtuais para estados excitados dos CT $[9,10]$.

Embora alguns estudos indiquem que a amplitude deste processo seja bem pequena [7], ele resulta no aparecimento do efeito Kondo, onde neste caso não são spins que estão correlacionados, mas os elétrons de condução e a partícula pesada que formam um estado ligado fortemente correlacionado do tipo Kondo [7,11,12].

$\mathrm{O}$ primeiro a propor um modelo para o problema de dois níveis acoplado aos elétrons de condução de um metal foi Kondo em um artigo de 1976 [1]. Neste artigo, Kondo estuda, através da técnica perturbativa, a taxa de tunelamento de um íon entre dois sítios de um metal hospedeiro. Denominaremos esse modelo de Modelo de Kondo de Tunelamento. Nele, os elétrons de condução ficam submetidos a um potencial do tipo Coulombiano, cujo centro é móvel. Esse acoplamento eletrostático faz com que os elétrons de condução tentem blindar o íon tunelante, resultando no aumento da massa efetiva do íon, em outras palavras, a taxa de tunelamento é renormalizada para valores menores que a taxa livre na qual não há interação banda/íon. Dizemos, nesse caso, que temos uma taxa efetiva de tunelamento.

Kondo não considerou a possibilidade de ocorrer o tunelamento assistido em seu trabalho por considerar o termo do Hamiltoniano que dá origem a ele muito pequeno. Trabalhos subsequentes de Kondo e, notadamente de Zawadowski e Zárand, mostram essa possibilidade [7,13].

Em nosso trabalho, estudamos o calor específico do modelo de Kondo de tunelamento acrescido do tunelamento assistido, sendo que para este propomos uma forma específica como veremos adiante. Também estudamos o comportamento da taxa efetiva de tunelamento em função do parâmetro que representa o tunelamento assistido.

O Hamiltoniano para esse modelo é de muitos corpos, o que exige técnicas sofisticadas para sua diagonalização. Neste trabalho utilizamos a técnica do Grupo de Renormalização Numérico (GRN) para sua solução. 


\section{2 - O Modelo}

O modelo que estamos analisando consiste de um íon sem spin, que tunela entre dois mínimos locais de potencial centrados nos sítios $\vec{R} / 2$ e $-\vec{R} / 2$, denominados sítios 1 e 2, respectivamente, de um metal amorfo. Esta situação pode ser visualizada na Figura 1.1, onde a Fig. 1.1.a representa a situação onde um íon na estrutura amorfa tem duas posições estáveis próximas uma da outra, enquanto que a Fig. 1.1.b descreve o potencial efetivo. Podemos associar dois estados quânticos, $\varphi_{1}$ e $\varphi_{2}$, localizados nos sítios 1 e 2 , respectivamente. A banda de condução (BC) do metal é considerada isotrópica e simétrica em relação ao nível de energia de Fermi $\left(\varepsilon_{F}\right)$ com largura $2 D$ e definimos $\varepsilon_{F}=0$. O íon tunelante interage com os elétrons de condução eletrostaticamente através de um potencial $G$ independente do momento. O modelo possui, ainda, uma hibridização $\Delta$ entre os estados quânticos do íon. É considerado, também, o processo de tunelamento assistido pelos elétrons de condução com uma constante de acoplamento $G_{a}$. Além disso, não há troca de cargas entre o íon tunelante e a banda de condução.

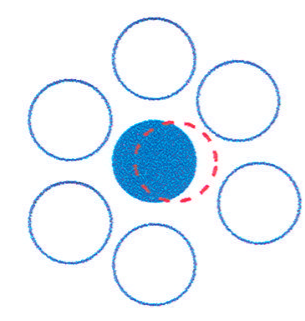

Fig. 1.1.a

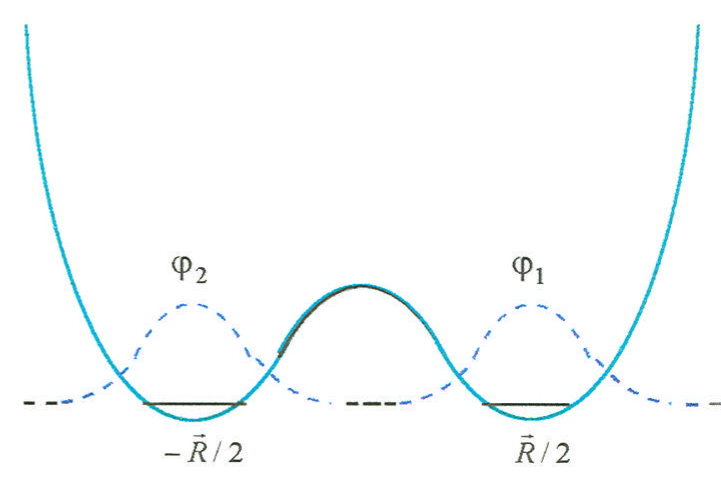

Fig. 1.1.b

Figura 1.1: a) Esquematização de um sistema de dois níveis em um metal amorfo; b) potencial efetivo que descreve seu movimento.

Para representar este modelo utilizamos o modelo de Kondo de Tunelamento somado ao termo de tunelamento assistido 


$$
H=H_{b c}+H_{t u n}+H_{e s p}+H_{a s s}
$$

onde $H_{b c}$ refere-se à $\mathrm{BC}, H_{t u n}$ ao tunelamento direto entre os mínimos, $H_{e s p}$ refere-se ao espalhamento dos elétrons de condução pelo íon e $H_{\text {ass }}$ é o termo que descreve o tunelamento assistido.

Para tratar esse problema, definimos os seguintes operadores fermiônicos: $c_{\vec{k}}$ destrói um elétron com momento $\vec{k}$ da $\mathrm{BC}, d_{1}\left(d_{2}\right)$ destrói o íon na posição $\vec{R} / 2(-\vec{R} / 2)$ e $\Psi(\vec{r})$ um operador de campo que destrói elétrons de condução na posição $\vec{r}$. Consideramos, ainda, os elétrons da $\mathrm{BC}$ como sendo livres. Com esses operadores podemos escrever $H_{b c}, H_{t u n}, H_{e s p}$ e $H_{a s s}$ da seguinte forma,

$$
\begin{gathered}
H_{b c}=\int d \vec{k} \varepsilon_{\vec{k}} c_{\vec{k}}^{+} c_{\vec{k}}, \\
H_{t u n}=-\frac{\Delta}{2}\left(d_{1}^{+} d_{2}+d_{2}^{+} d_{1}\right), \\
H_{e s p}=G \Psi^{+}(\vec{R} / 2) \Psi(\vec{R} / 2) d_{1}^{+} d_{1}+G \Psi^{+}(-\vec{R} / 2) \Psi(-\vec{R} / 2) d_{2}^{+} d_{2} \\
H_{\text {ass }}=G_{a} \Psi^{+}(\vec{R} / 2) \Psi(-\vec{R} / 2) d_{2}^{+} d_{1}+G_{a} \Psi^{+}(-\vec{R} / 2) \Psi(\vec{R} / 2) d_{1}^{+} d_{2},
\end{gathered}
$$

onde $\varepsilon_{\vec{k}}$ em $H_{b c}$ é a energia do elétron livre que depende apenas do módulo do momento $\vec{k}$ uma vez que a $\mathrm{BC}$ é considerada isotrópica. A composição de operadores $d_{1}^{+} d_{2}$, na Eq. (1.3), representa o tunelamento do íon do sítio 2 para 1 , sendo $d_{2}^{+} d_{1}$ o processo inverso. A constante $\Delta$ é denominada de taxa de tunelamento livre, ou seja, quando não há o acoplamento $G$ e $G_{a}$ entre o íon e a BC. O primeiro termo de $H_{\text {esp }}$ representa o espalhamento dos elétrons de condução quando o íon está na posição 1, sendo que o segundo termo tem interpretação equivalente. Em $H_{\text {ass }}$ temos novamente o termo de tunelamento $d_{2}^{+} d_{1}$ (primeiro termo do lado direito), mas agora associado com operadores de campo, onde um destrói elétrons da 
$\mathrm{BC}$ na posição 2 e o outro constrói na posição 1. Para o segundo termo temos interpretação análoga.

É facil perceber que o Hamiltoniano desse modelo não é trivial de ser diagonalizado, devido aos muitos graus de liberdade da BC. Além disso, os termos de espalhamento e tunelamento assistido são de natureza quártica, o que exige técnicas de muitos corpos. O método utilizado nesta dissertação foi o Grupo de Renormalização Numérico (GRN). Falaremos brevemente deste método a seguir.

\section{3 - Grupo de Renormalização Numérico}

O GRN foi introduzido por Wilson em 1975 [14] para o estudo de impurezas magnéticas diluídas em metal (Modelo de Kondo, que não deve ser confundido com o Modelo de Kondo de Tunelamento descrito na seção anterior), e resume-se na identificação sistemática das escalas de energia do problema. Em seguida, foi aplicado ao estudo de propriedades estáticas do modelo de Anderson por Krishna-Murthy et. al. $[15]$.

Originalmente havia dificuldades de se aplicar essa técnica ao estudo de propriedades dinâmicas como densidade espectral do modelo de Anderson ou mesmo de propriedades termodinâmicas como o calor específico, no qual a técnica introduzia muitas oscilações na curva desta propriedade.

Essas dificuldades foram superadas através de aperfeiçoamentos da técnica introduzidos por L. N. Oliveira et. al. [16], os quais permitem que exploremos as escalas de energia do problema com maior precisão.

O GRN é um método para diagonalizar Hamiltonianos de sistemas com várias escalas de energia. De modo geral, o método consiste em diagonalizar um Hamiltoniano de maneira recursiva, considerando parte dos graus de liberdade de cada vez, sendo que em cada etapa os parâmetros do problema são reescalonados. Em cada passo, com os autovalores e autovetores do Hamiltoniano atual se constrói as bases para o Hamiltoniano seguinte, que será diagonalizado, dando sequência ao processo, o qual termina quando temos a precisão desejada.

Uma parte importante do método é a discretização logarítmica do espectro de energia. Em nosso modelo, o espectro é dado pela BC do metal, sendo que as 
interações de interesse são da ordem de $\Delta$ e estão próximas do nível de Fermi, região enfatizada pela discretização logarítmica.

O modelo de Kondo de tunelamento já vem sendo estudado através da técnica do GRN há algum tempo. Por exemplo, Líbero e J. Vítor estudaram o calor específico [17] e Líbero e L. N. Oliveira estudaram a densidade espectral desse problema [18]. Por isso, seguimos os passos introduzidos por esses tabalhos para diagonalizar nosso Hamiltoniano. Feita a diagonalização, podemos calcular o calor específico do modelo. Na próxima seção, mostraremos alguns resultados obtidos para essa propriedade.

\section{4 - Resumo dos Resultados}

O modelo que trabalhamos tem como parâmetros iniciais os segintes: $G$, que indica a intensidade da interação Coulombiana; $G_{a}$, que dá a intensidade do tunelamento assistido; $\Delta$, que é a taxa de tunelamento livre (quando $G=0$ e $G_{a}=0$ ) e $S$, que é definido pela expressão $S=\operatorname{sen}\left(k_{F} R\right) / k_{F} R$ e indica o afastamento entre os mínimos de potencial, sendo $k_{F}$ o módulo do momento do elétron de condução no nivel de Fermi. A escolha adequada destes parâmetros (basicamente $\Delta=0$ e $S=0$ ) nos permite mapear o Hamiltoniano de Kondo de Tunelamento, que é o Hamiltoniano de interesse nesse trabalho, no Hamiltoniano de Kondo, sendo que este descreve o problena de uma impureza magnética em metal (falaremos um pouco mais sobre o Modelo de Kondo na seção 4.4). A Fig. 1.2 mostra o calor específico em função da temperatura para essa escolha de parâmetros e a estamos comparando com a curva universal de calor específico do modelo de Kondo. Podemos observar a excelente concordância entre nosso resultado e a curva universal.

Quando estamos com todos os parâmetros diferentes de zero as curvas de calor específico se comportam como mostra a Fig. 1.3, quando aumentamos $G_{a}$ a partir de $G_{a}$ igual a zero. Observe que as curvas se deslocam para baixas temperaturas até atingir uma certa região e, então, se deslocam para temperaturas mais altas. Este comportamento mostra que a taxa efetiva de tunelamento do íon diminui e depois aumenta, uma vez que a temperatura para a qual ocorre o pico do 
calor específico é relacionada com essa taxa. No Capítulo 4 mostraremos que se $G$ for positivo o comportamento das curvas é o mesmo que a mostrada aqui.

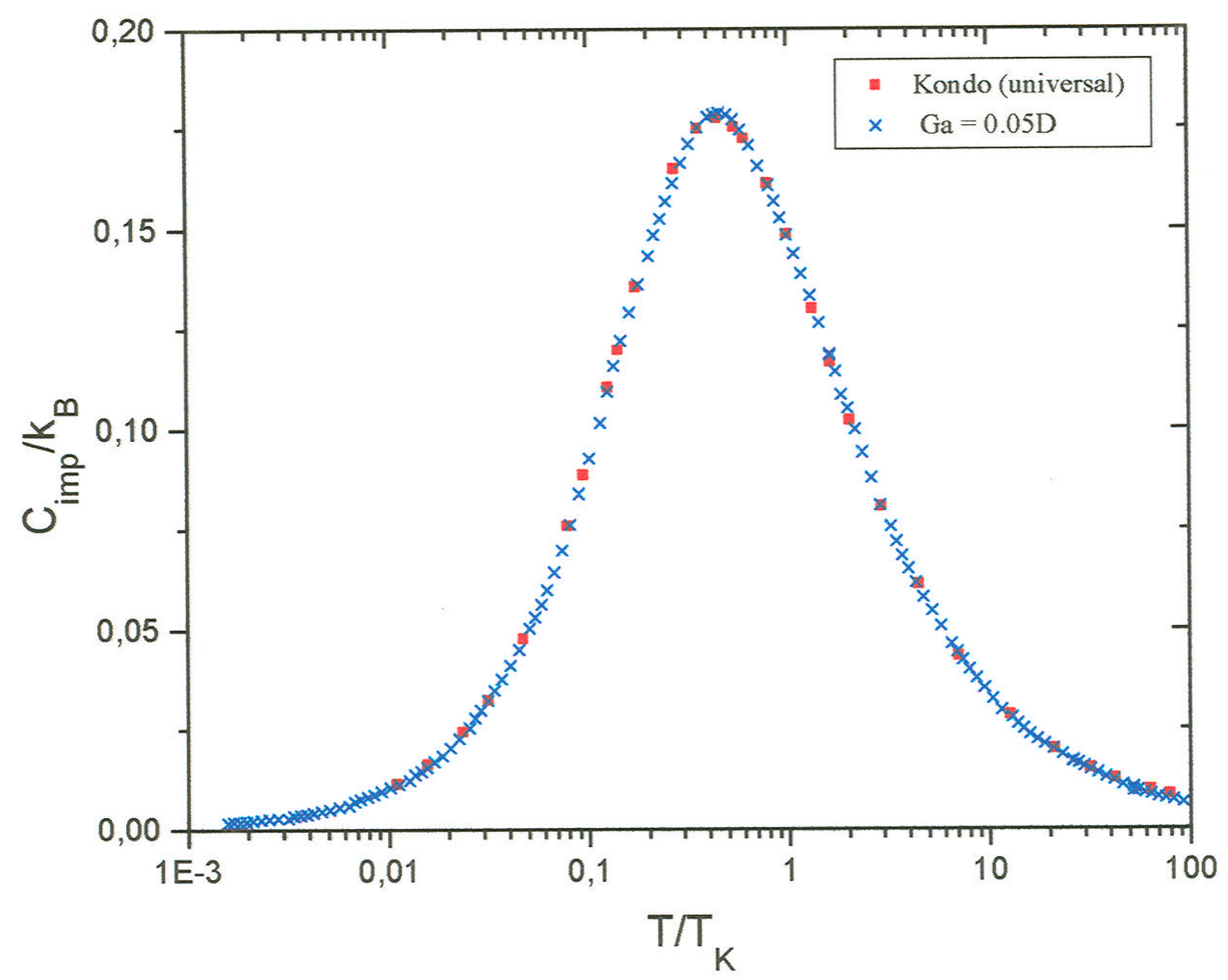

Figura 1.2: A curva universal do calor específico do modelo de Kondo é representada nesta figura pelos pontos vermelhos. Os pontos azuis representam o calor específico calculado com nossa escolha de parâmetros que faz com que nosso Hamiltoniano seja mapeado no Hamiltoniano do Modelo de Kondo. A temperatura de Kondo, $T_{K}$, foi utilizada para normalizar a curva. Esta é a temperatura que separa as regiões de acoplamento fraco e forte no modelo de Kondo de impurezas magnéticas diluídas em metal (ver seção 4.4)

\section{5 - Organização Geral do Trabalho}

No Capítulo 2 nos aprofundaremos no estudo do Modelo de Kondo de Tunelamento, detalhando a aproximação que Kondo fez. Vamos comparar a expressão proposta por nós para o tunelamento assistido com a forma geral de interação entre um sistema de dois níveis e elétrons de condução mostrada por Zawadowski, concluíndo que nosso Hamiltoniano respeita essa estrutura. 


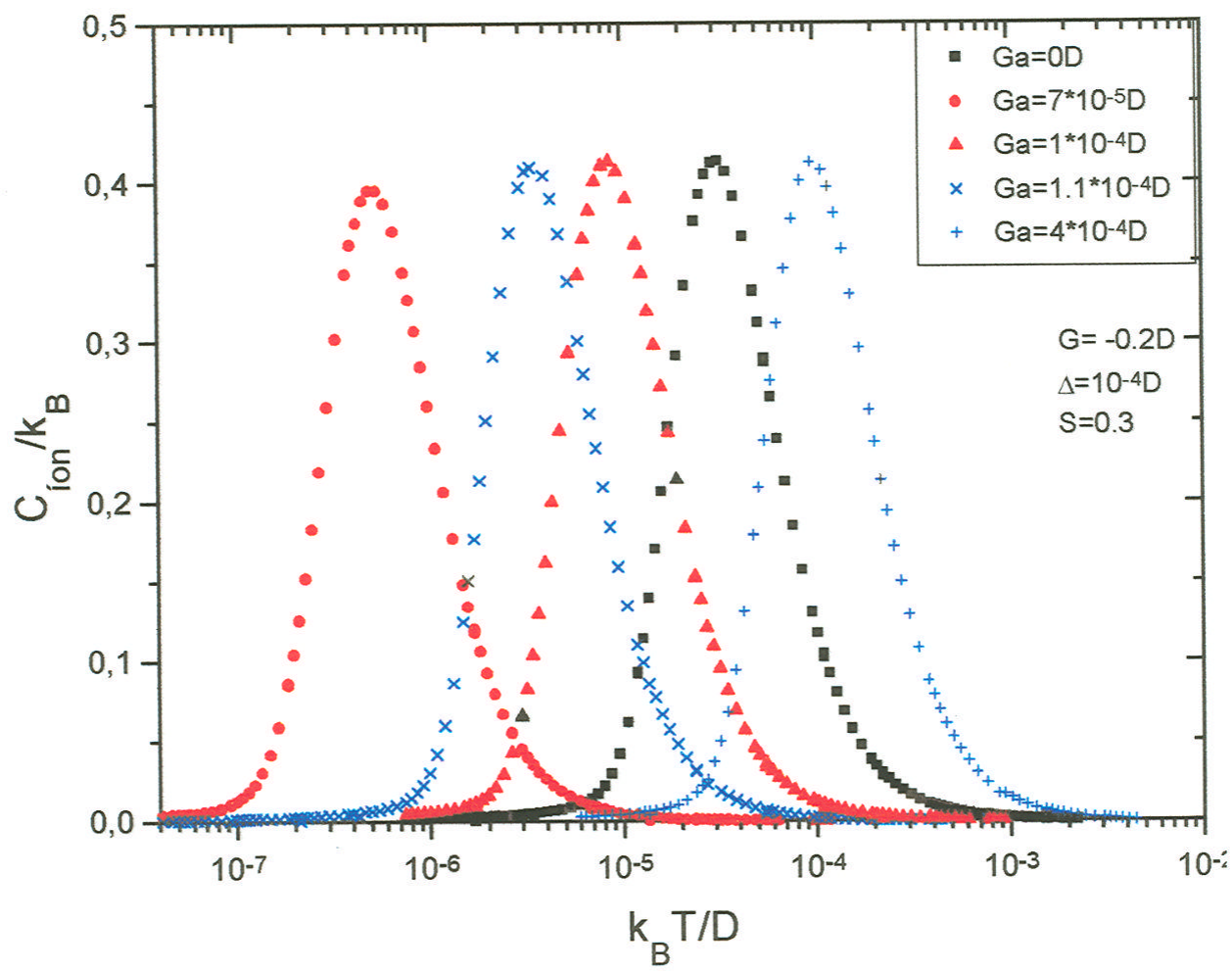

Figura 1.3: Comportamento de várias curvas de calor específico quando variamos o parâmetro de tunelamento assistido. Observa-se que a curva se desloca para baixas temperaturas quando aumentamos esse parâmetro (as curvas vermelhas representam este caso), e então, volta para altas temperaturas (as curvas azuis ilustram essa situação)

O Capítulo 3 é extremamente técnico. Nele mostraremos a adaptação do Hamiltoniano ao GRN, definindo uma transformação do grupo de renormalização de modo a poder tratá-lo numericamente.

Os resultados numéricos são apresentados no Capítulo 4, onde mostramos a influência do tunelamento assistido no calor específico e na taxa de tunelamento. No capítulo 4, detalharemos, também, o mapeamento do Hamiltoniano estudado por nós no Hamiltoniano de Kondo de uma impureza magnética em metais. O capítulo 5 é dedicado à conclusões gerais e sugestões para futuros trabalhos. 


\section{Capítulo 2}

\section{O Modelo de Kondo de Tunelamento}

O modelo analisado por Kondo em um artigo publicado em 1976 [1] é o de um íon sem spin que pode tunelar entre dois sítios equivalentes de um metal (dois mínimos locais de potencial) e que interage eletrostaticamente com os elétrons da BC. Os sítios estão distanciados de $\vec{R}$, sendo que a origem de coordenadas é adotada a meia distância entre os sítíos, para que se possa explorar a simetria de inversão do problema. A Figura 2.1 mostra uma representação esquemática do modelo.

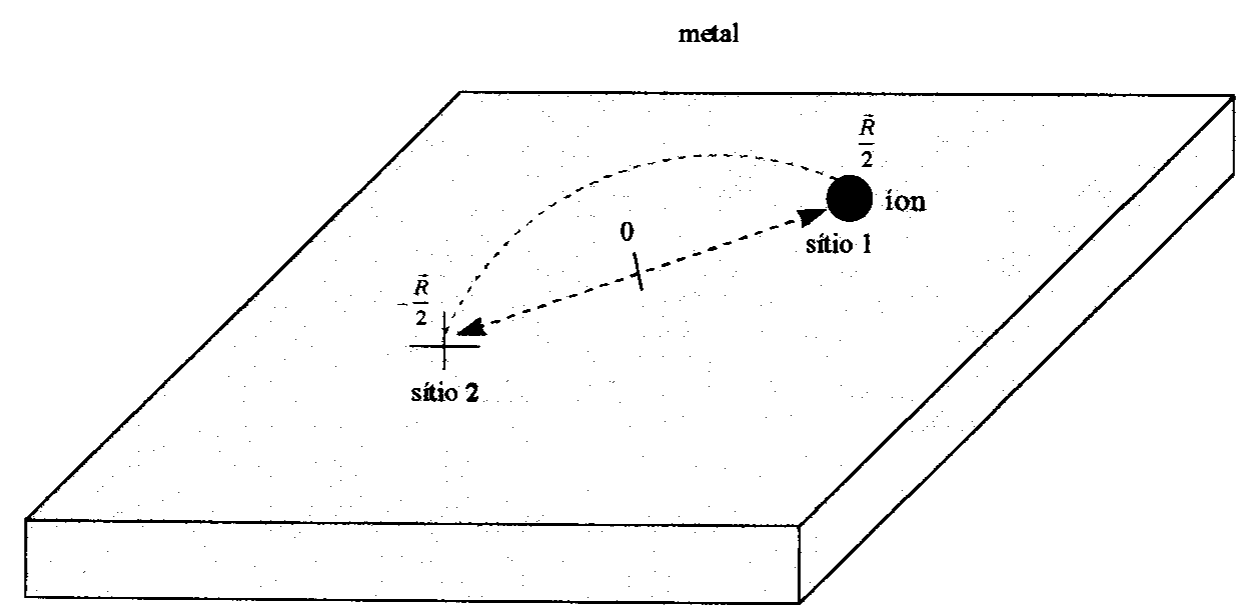

Figura 2.1: Representação esquemática de um íon num metal tunelando entre dois sítios equivalentes. Os sítios estão separados pela distância $R$ sendo a origem do sistema a meia distância entre os sítios. A linha tracejada curva representa o íon tunelando entre os sítios 1 e 2 .

A cada mínimo local é associado, para $o$ íon, uma função de onda: $\varphi_{1}$ centrado no sítio 1 e $\varphi_{2}$ centrado no sítio 2, ambos de mesma energia (Figura 2.2-a). Considerando que as funções de onda se hibridizam, teremos o aparecimento de um estado de mais baixa energia, ligante, e um de mais alta energia, antiligante, sendo $\Delta$ a diferença de energia entre eles (Figura 2.2-b). 
Como estamos mais interessados em estudar o sistema em baixas temperaturas, consideramos que o movimento pode ocorrer apenas por tunelamento e não através de "saltos" via ativação térmica de um sítio para outro.

Devido à interação Coulombiana os elétrons da $\mathrm{BC}$ são espalhados. Se essa interação não existisse, a freqüência com que o íon tunelaria de um sítio para outro seria $\Delta / h$ (onde $\Delta$ é a taxa de tunelamento livre), porém a presença da interação diminui o valor dessa taxa, que pode ser interpretada como devido a um aumento da massa do íon tunelante.

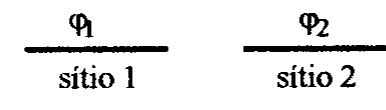

(a)

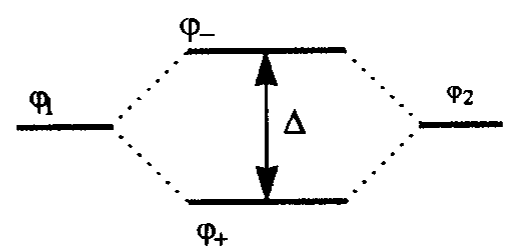

(b)

Figura 2.2: Representação esquemática do problema de dois níveis localizados em um metal. (a) Cada mínimo local é representado por uma função de onda: $\varphi_{1}$ para o mínimo do sítio 1 e $\varphi_{2}$ para o sítio 2. (b) Abertura da degenerescência dos níveis localizados em um estado ligante $\left(\varphi_{+}\right)$e um antiligante $\left(\varphi_{-}\right)$, separados por uma energia $\Delta$.

No modelo de Kondo foi considerado apenas o tunelamento direto, ou seja, aquele vindo da superposição das funções de onda dos orbitais $\varphi_{1}$ e $\varphi_{2}$. Contudo, a possibilidade do tunelamento ocorrer através do espalhamento dos elétrons de condução existe como apontado pelo próprio Kondo e depois por Zawadowski et. al. $[7,13]$. Esse é o processo de tunelamento assistido pelos elétrons de condução, ou simplesmente tunelamento assistido.

O Hamiltoniano de Kondo de tunelamento para esse sistema pode ser escrito como:

$$
H=H_{b c}+H_{\text {tun }}+H_{\text {int }},
$$

sendo $H_{b c}$ referente à $\mathrm{BC}, H_{t u n}$ ao tunelamento direto do íon entre os mínimos e $H_{\text {int }}$ é o termo de interação Coulombiana entre o íon tunelante e os elétrons da BC. 


\section{1 - Analisando os Termos de $H$.}

Nesta seção vamos analisar cada um dos termos que aparecem na Eq.(2.1).

\subsection{1 - Hamiltoniano da Banda de Condução}

Os elétrons da BC são assumidos como livres e representados por $\exp (i \vec{k} \cdot \vec{r})$, onde $\vec{k}$ é o momento do elétron. Dessa forma, o termo $H_{b c}$, que é o Hamiltoniano que descreve o metal em si, é escrito em linguagem de segunda quantização como:

$$
H_{b c}=\int d \vec{k} \varepsilon_{\vec{k}} c_{\vec{k}}^{+} c_{\vec{k}}
$$

onde $c_{\vec{k}}$ é o operador de aniquilamento de um elétron de condução com momento $\vec{k}$ e energia cinética $\varepsilon_{\vec{k}}$. Assim, $H_{b c}$ nos dá a energia cinética total da BC. Nesse modelo, consideramos a $\mathrm{BC}$ do metal isotrópica e simétrica em relação ao nível de energia de Fermi $\left(\varepsilon_{F}\right)$, com largura $2 D$ e definimos $\varepsilon_{F}=0$ (Figura 2.3). É importante salientar que não há troca de elétrons entre o íon e a $\mathrm{BC}$. Assim, o número de elétrons desta mantém-se constante, o mesmo acontecendo com a carga total do íon que permanece igual à unidade.

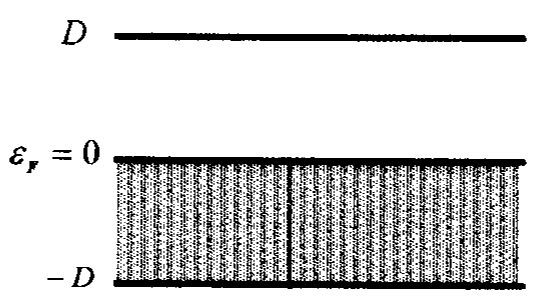

Figura 2.3: Representação esquemática da banda de condução do metal. A banda é isotrópica e simétrica em relação ao nível de Fermi $\left(\varepsilon_{F}\right)$ e com largura $2 D$.

\subsection{2 - Hamiltoniano de Tunelamento Direto}

O termo $H_{\text {tun }}$ representa o tunelamento direto do íon, originado da superposição de suas funções de onda quando ele tunela de um sítio para outro, e é representado por 


$$
H_{\text {tun }}=-\frac{\Delta}{2}\left(d_{1}^{+} d_{2}+d_{2}^{+} d_{1}\right)
$$

sendo $d_{1}\left(d_{2}\right)$ o operador de destruição do ín no sítio 1(2). Esse termo dá origem a um sistema de dois níveis, onde temos um estado de mais baixa energia (ligante) representado por $\varphi_{+}$, e um de mais alta energia (antiligante) representado por $\varphi_{-}$, separados pela energia $\Delta$ (Fig. 2.2-b).

\subsection{3 - Hamiltoniano de Interação}

No desenvolvimento do Hamiltoniano de interação, $H_{\text {int }}$, para esse problema, Kondo considerou que a interação entre o íon e os elétrons de condução é local e puramente Coulombiana, isto é, só depende das suas coordenadas relativas, $V\left(\vec{r}-\vec{r}_{a}\right)$. Considerou, também, que os elétrons da BC, como já apontado, são livres e representados por $\exp (\vec{i} \cdot \vec{r})$, onde $\vec{k}$ é o momento do elétron. A função de onda do íon foi considerada como sendo localizada na posição $\vec{r}_{a}$ e no estado quântico $\alpha$ denominando-a $\varphi_{\alpha}\left(\vec{r}_{a}\right)$, sendo que este é o auto-estado do íon que pode ser o estado ligante, $\varphi_{+}\left(\vec{r}_{a}\right)$, ou antiligante, $\varphi_{-}\left(\vec{r}_{a}\right)$. Dessa forma, a base para escrever o termo de interação é dada por $\left|\varphi_{\alpha} ; \vec{k}\right\rangle$, onde $\vec{k}$ representa os elétrons de condução. Assim, $H_{\text {int }}$ é escrito como [19]

$$
H_{\text {int }}=\sum_{\alpha \alpha^{\prime} \vec{k} \vec{k}^{\prime}}\left\langle\varphi_{\alpha^{\prime}} ; \vec{k},\left|V\left(\vec{r}-\vec{r}_{\alpha}\right)\right| \varphi_{\alpha} ; \vec{k}\right\rangle d_{\alpha^{\prime}}^{+} d_{\alpha} c_{\vec{k}}^{+}, c_{\vec{k}}
$$

sendo $d_{\alpha}$ o operador que destrói o íon no estado $\alpha(\alpha=+,-)$. No Hamiltoniano, o inverso do volume do material deveria aparecer, mas para não sobrecarregar a notação, adotamos o seu valor como sendo unitário.

$O$ elemento de matriz é dado por

$$
\begin{aligned}
& \left\langle\varphi_{\alpha}^{*},\left(\vec{r}_{a}\right) ; \vec{k}^{\prime}\left|V\left(\vec{r}-\vec{r}_{a}\right)\right| \varphi_{\alpha}\left(\vec{r}_{a}\right) ; \vec{k}\right\rangle= \\
& \int e^{-i \vec{k}, \vec{r}} \varphi_{\alpha^{\prime}}^{*}\left(\vec{r}_{a}\right) V\left(\vec{r}-\vec{r}_{a}\right) e^{i \vec{k} \cdot \vec{r}} \varphi_{\alpha}\left(r_{a}\right) d^{3} r d^{3} r_{a} \equiv \\
& V\left(\vec{k}^{\prime}-\vec{k}\right) J_{\alpha^{\prime} \alpha}(\vec{k},-\vec{k}),
\end{aligned}
$$


sendo $V(\vec{k},-\vec{k})$ a transformada de Fourier do potencial Coulombiano e $J_{\alpha^{\prime} \alpha}\left(\vec{k}^{\prime}-\vec{k}\right)$ definido por

$$
J_{\alpha^{\prime} \alpha}(\vec{k},-\vec{k})=\int e^{i\left(\vec{k}-\vec{k}^{\prime}\right) \cdot \vec{r}_{a}} \varphi_{\alpha^{\prime}}^{*}\left(\vec{r}_{a}\right) \varphi_{\alpha}\left(\vec{r}_{a}\right) d^{3} r_{\alpha}
$$

Observe que em (2.5) temos uma integração envolvendo a coordenada iônica $\vec{r}_{a}$ (visto que as funções $\varphi_{\alpha}\left(\vec{r}_{a}\right)$ podem, em geral, ter uma certa largura em torno dos sítios $-\vec{R} / 2$ e $\vec{R} / 2$, sendo estes os sítios que podem ser ocupados pelo íon), e outra na coordenada eletrônica $\vec{r}$.

Tendo o íon apenas dois estados possíveis (ligante e antiligante) como base, Kondo utilizou as matrizes de Pauli para expressá-los. Dessa forma, definindo

$$
\sigma_{x}=d_{+}^{+} d_{-}+d_{-}^{+} d_{+} \quad, \quad \sigma_{z}=d_{+}^{+} d_{+}-d_{-}^{+} d_{-} \quad \mathrm{e} \quad \mathrm{I}=d_{+}^{+} d_{+}+d_{-}^{+} d_{-},
$$

onde I é a matriz identidade, $H_{\text {int }}$ pode ser expresso como:

$$
H_{\text {int }}=\sum_{\vec{k} \vec{k}}\left(L_{\vec{k}, \vec{k}} \mathrm{I}+M_{\vec{k}, \vec{k}} \sigma_{z}+N_{\vec{k}, \vec{k}} \sigma_{x}\right) c_{\vec{k}}^{+}, c_{\vec{k}}
$$

sendo

$$
\begin{aligned}
& L_{\vec{k}, \vec{k}}=V\left(\vec{k}^{\prime}-\vec{k}\right) \frac{J_{++}\left(\vec{k}^{\prime}-\vec{k}\right)+J_{--}\left(\vec{k}^{\prime}-\vec{k}\right)}{2}, \\
& M_{\vec{k}, \vec{k}}=V\left(\vec{k}^{\prime}-\vec{k}\right) \frac{J_{++}\left(\vec{k}^{\prime}-\vec{k}\right)-J_{--}\left(\vec{k}^{\prime}-\vec{k}\right)}{2} \\
& \mathrm{e} \\
& N_{\vec{k}, \vec{k}}=V\left(\vec{k}^{\prime}-\vec{k}\right) J_{-+}\left(\vec{k}^{\prime}-\vec{k}\right) .
\end{aligned}
$$

Para simplificar $H_{\text {int }}$, Kondo representou $\varphi_{+}\left(\vec{r}_{a}\right)$ e $\varphi_{-}\left(\vec{r}_{a}\right)$ em termos das funções de Wannier $\phi_{1}$ e $\phi_{2}$, localizadas nas posições $\vec{R} / 2$ e $-\vec{R} / 2$, respectivamente,

$$
\varphi_{+}\left(\vec{r}_{a}\right)=\frac{\phi_{1}\left(\vec{r}_{a}\right)+\phi_{2}\left(\vec{r}_{a}\right)}{\sqrt{2}} \quad \text { e } \quad \varphi_{-}\left(\vec{r}_{a}\right)=\frac{\phi_{1}\left(\vec{r}_{a}\right)-\phi_{2}\left(\vec{r}_{a}\right)}{\sqrt{2}}
$$


Considerando $\phi_{1}$ e $\phi_{2}$ bem localizadas, ou seja,

$$
\phi_{1}=\delta\left(\vec{r}_{\alpha}-\frac{\vec{R}}{2}\right) \quad \text { e } \quad \phi_{2}=\delta\left(\vec{r}_{\alpha}+\frac{\vec{R}}{2}\right)
$$

as Eqs. (2.9), após algumas manipulações matemáticas simples, ficam

$$
\begin{aligned}
& L_{\vec{k}, \vec{k}}=\frac{V(\vec{k},-\vec{k})}{2}\left(e^{i(\vec{k}-\vec{k}) \cdot \vec{R} / 2}+e^{-i\left(\vec{k}-\vec{k}^{\prime}\right) \cdot \vec{R} / 2}\right), \\
& N_{\vec{k}, \vec{k}}=\frac{V\left(\vec{k}^{\prime}-\vec{k}\right)}{2}\left(e^{i(\vec{k}-\vec{k}, \cdot) \cdot R / 2}-e^{-i(\vec{k}-\vec{k}) \cdot \vec{R} / 2}\right) \\
& \mathrm{e} \\
& M_{\vec{k}, \vec{k}}=V(\vec{k},-\vec{k}) \int e^{i\left(\vec{k}-\vec{k}^{\prime}\right) \cdot \vec{R} / 2} \phi_{1}\left(\vec{r}_{\alpha}\right) \phi_{2}\left(\vec{r}_{\alpha}\right) d^{3} r_{\alpha} .
\end{aligned}
$$

Substituindo as expressões (2.12) na Eq. (2.8) obtemos

$$
H_{e s p}=\sum_{\vec{k} \vec{k}} V(\vec{k},-\vec{k})\left(e^{i(\vec{k}-\vec{k},) \cdot \vec{R} / 2} d_{1}^{+} d_{1}+e^{-i(\vec{k}-\vec{k},) \cdot \vec{R} / 2} d_{2}^{+} d_{2}\right) c_{\vec{k}}^{+}, c_{\vec{k}},
$$

onde mudamos a especificação $H_{\text {int }}$ por $H_{\text {esp }}$ e utilizamos as transformações com paridade definida dos operadores $d_{+}$e d como segue

$$
d_{+}=\frac{d_{1}+d_{2}}{\sqrt{2}} \quad \mathrm{e} \quad d_{-}=\frac{d_{1}-d_{2}}{\sqrt{2}}
$$

para podermos escrever a Eq. (2.13) em função de $d_{1}^{+} d_{1}$ e $d_{2}^{+} d_{2}$.

Observe que nas Eqs. (2.12) o termo $M_{\vec{k}, \vec{k}}$ foi deixado em forma de integral e representaria, segundo Kondo, o espalhamento dos elétrons de condução seguido de um salto do íon do sítio 1 para 2, ou vice-versa, por estar associado à $\sigma_{z}$ na Eq. (2.8). Caso $\phi_{1}$ e $\phi_{2}$ não fossem funções localizadas esse termo desempenharia o papel de tunelamento assistido. Contudo, esse termo foi desprezado por Kondo por se 
tratar do produto de duas funções deltas centradas em posições diferentes, portanto seu valor é nulo.

O termo $L_{\vec{k}, \vec{k}}$ nas Eqs. (2.12) também foi desconsiderado por Kondo em seu cálculo perturbativo da taxa de tunelamento. Isso porque esse termo está associado à identidade na Eq.(2.8) e esta não é responsável pelo tunelamento do íon de um sítio ao outro. Seguindo a mesma linha de trabalho de João Vítor e Líbero [17], diagonalizamos o Hamiltoniano de tunelamento sem excluir esse termo $L_{\vec{k} \cdot \vec{k}}$ (a Eq. (2.13) já leva em conta este fato) utilizando o GRN.

A blindagem eletrostática dos elétrons da $\mathrm{BC}$ em torno do íon tunelante faz com que o potencial Coulombiano efetivo seja de curto alcance, e por isso, podemos considerar sua transformada de Fourier constante,

$$
V(\vec{k},-\vec{k})=G
$$

Definindo o operador de campo

$$
\Psi(\vec{r})=\sum_{\vec{k}} e^{i \vec{k} \cdot \vec{r}} c_{\vec{k}}
$$

que destrói elétrons da $\mathrm{BC}$ no sítio $\vec{r}$, podemos escrever, finalmente, $H_{e s p}$ como

$$
H_{e s p}=G \Psi^{+}(\vec{R} / 2) \Psi(\vec{R} / 2) d_{1}^{+} d_{1}+G \Psi^{+}(-\vec{R} / 2) \Psi(-\vec{R} / 2) d_{2}^{+} d_{2}
$$

Portanto, esse é o termo que descreve o espalhamento dos elétrons da $\mathrm{BC}$ através do potencial de Coulomb quando o íon está na posição 1 , ou na posição 2, daí vem a preferência de se escrever $H_{\text {esp }}$ ao invés de $H_{\text {int }}$.

\section{2 - Hamiltoniano de Tunelamento Assistido}

Para o tunelamento assistido $H_{\text {ass }}$, estamos propondo o seguinte Hamiltoniano 


$$
\left.\left.H_{a s s}=\sum_{\vec{k} \vec{k}} V(\vec{k},-\vec{k})\left(e^{-i(\vec{k}+\vec{k},}\right) \cdot \vec{R} / 2 d_{2}^{+} d_{1}+e^{i(\vec{k}+\vec{k},}\right) \cdot \vec{R} / 2 d_{1}^{+} d_{2}\right) c_{\vec{k}}^{+}, c_{\vec{k}},
$$

Neste caso em que há troca de posição do íon enquanto os elétrons de condução são espalhados, a blindagem eletrostática dos elétrons da $\mathrm{BC}$ ao redor do íon tunelante é diferente da que ocorre no termo de espalhamento, Eq. (2.17). Por isso, podemos considerar $V(\vec{k},-\vec{k})=G_{a}$.

$H_{\text {ass }}$ pode, então, ser escrito em termos de $G_{a}$ e de $\Psi(\vec{r})$, e obtemos, assim

$$
H_{\text {ass }}=G_{a} \Psi^{+}(\vec{R} / 2) \Psi(-\vec{R} / 2) d_{2}^{+} d_{1}+G_{a} \Psi^{+}(-\vec{R} / 2) \Psi(\vec{R} / 2) d_{1}^{+} d_{2}
$$

A Figura 2.4 ilustra esse processo de tunelamento. Na Figura 2.4-a (correspondente ao primeiro termo do lado direito da Eq. (2.19)), a linha reta representa o íon tunelando do sítio 1 para o sítio 2, enquanto que a linha curva representa a nuvem eletrônica da $\mathrm{BC}$ se deslocando do sítio 2 para 1. A Figura 2.4-b representa o processo contrário e corresponde ao segundo termo do lado direito da Eq. (2.19).
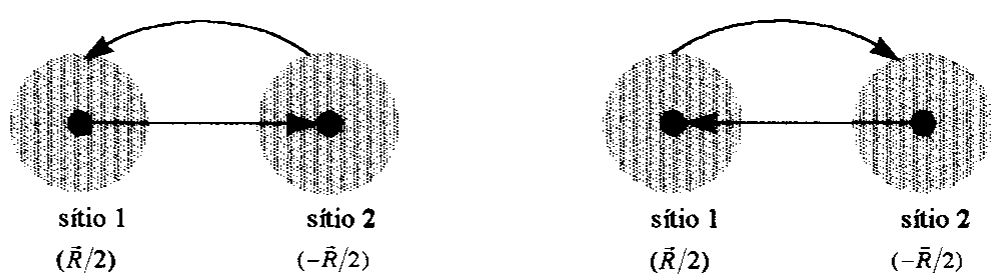

Figura 2.4: Espalhamento dos elétrons de condução pelo íon no processo de tunelamento assistido. (a) o íon (círculo vermelho) tunela da posição 1 para 2 enquanto os elétrons de condução (círculo azul) são espalhados da posição 2 para 1. (b) aqui mostramos a sequência inversa do processo.

Observe que esse tunelamento é influenciado pela $\mathrm{BC}$, ao contrário do tunelamento $\Delta$ que independe da BC.

\section{3 - A Soma de $H_{\text {esp }}+H_{\text {ass }}$}

Queremos, neste ponto, comparar a soma do termo de espalhamento com o tunelamento assistido $\left(H_{e s p}+H_{\text {ass }}\right)$ com o termo de interação geral mostrado por 
Zawadowski e outros [7,11]. Para isso, vamos utilizar as Eqs. (2.13) e (2.18) para expressar $H_{\text {esp }}+H_{\text {ass }}$, obtendo dessa forma

$$
\begin{aligned}
H_{e s p}+H_{a s s}= & \sum_{\vec{k} \vec{k}}\left[G e^{i(\vec{k}-\vec{k}) \cdot \vec{R} / 2} d_{1}^{+} d_{1}+G e^{-i(\vec{k}-\vec{k}) \cdot \vec{R} / 2} d_{2}^{+} d_{2}\right. \\
& \left.G_{a} e^{-i(\vec{k}+\vec{k},) \cdot \vec{R} / 2} d_{2}^{+} d_{1}+G_{a} e^{i(\vec{k}+\vec{k}) \cdot \vec{R} / 2} d_{1}^{+} d_{2}\right] c_{\vec{k}}^{+}, c_{\vec{k}} .
\end{aligned}
$$

A soma acima pode ser reescrita em função das matrizes de Pauli , Eqs. (2.7), obtendo

$$
\begin{aligned}
H_{e s p}+H_{a s s}= & \sum_{\vec{k} \vec{k}^{\prime}}\left\{G \cos \left[\left(\vec{k}^{\prime}-\vec{k}\right) \cdot \vec{R} / 2\right] I+i G \operatorname{sen}\left[\left(\vec{k}^{\prime}-\vec{k}\right) \cdot \vec{R} / 2\right] \sigma_{x}+\right. \\
& \left.G_{a} \operatorname{sen}\left[\left(\vec{k}^{\prime}+\vec{k}\right) \cdot \vec{R} / 2\right] \sigma_{y}+G_{a} \cos \left[\left(\vec{k}^{\prime}+\vec{k}\right) \cdot \vec{R} / 2\right] \sigma_{z}\right\} c_{\vec{k},}^{+}, c_{\vec{k}},
\end{aligned}
$$

onde $\sigma_{y}=i\left(d_{-}^{+} d_{+}-d_{+}^{+} d_{-}\right)$.

Os termos associados a $I$ e $\sigma_{x}$ na Eq. (2.21) correspondem à $L_{\vec{k}, \vec{k}}$ e $M_{\vec{k}, \vec{k}}$, respectivamente, nas Eqs. (2.12).

A forma geral da interação entre elétrons de condução e um sistema de dois níveis mostrada por Zawadowski é:

$$
H_{I}^{\prime}=\sum_{\substack{k, k \\ \alpha \beta}} d_{\beta}^{+} c_{\vec{k}}^{+}, V_{\vec{k}, \vec{k}}^{\beta \alpha} c_{\vec{k}} d_{\alpha},
$$

sendo que $\alpha$ e $\beta$ se referem aqui aos orbitais ligante e antiligante e podem tomar os valores + ou - . Expressando $H_{I}^{\prime}$ em termos das matrizes de Pauli ${ }^{1}$, obtemos

$$
H_{I}^{\prime}=\sum_{\vec{k} \vec{k}}\left[V_{\vec{k}, \vec{k}}^{0} \mathrm{I}+V_{\vec{k}, \vec{k}}^{x} \sigma_{x}+V_{\vec{k}, \vec{k}}^{y} \sigma_{y}+V_{\vec{k}, \vec{k}}^{z} \sigma_{z}\right] c_{k}^{+}, c_{\vec{k}} \cdot
$$

\footnotetext{
${ }^{1}$ A notação utilizada por Zawadowski em seu artigo para escrever as matrizes de Pauli é diferente da utilizada por Kondo, sendo esta a utilizada nesta dissertação. Isso faz com que nossos termos

$V_{\vec{k}, \vec{k}}^{0}, V_{\vec{k}, \vec{k}}^{x}, V_{\vec{k}, \vec{k}}^{y}$ e $V_{\vec{k}, \vec{k}}^{z}$ correspondam no artigo de Zawadowski à $V_{\vec{k}, \vec{k}}^{0}, V_{\vec{k}, \vec{k}}^{z},-V_{\vec{k}, \vec{k}}^{y}$ e $V_{\vec{k}, \vec{k}}^{x}$, respectivamente.
} 
Zawadowski propõe uma expressão apenas para $V_{\vec{k}, \vec{k}}^{x}$ em seu trabalho. Podemos, contudo, comparar a expressão (2.23) com nossa soma $H_{\text {esp }}+H_{\text {ass }}$, Eq. (2.21), e fazer a seguinte associação:

$$
\begin{aligned}
V_{\vec{k}, \vec{k}}^{0} & =G \cos \left(\vec{k}^{\prime}-\vec{k}\right) \cdot \vec{R} / 2, \\
V_{\vec{k}, \vec{k}}^{x} & =i G \operatorname{sen}\left(\vec{k}^{\prime}-\vec{k}\right) \cdot \vec{R} / 2, \\
V_{\vec{k}, \vec{k}}^{y} & =G_{a} \operatorname{sen}\left(\vec{k}^{\prime}+\vec{k}\right) \cdot \vec{R} / 2 \mathrm{e} \\
V_{\vec{k}, \vec{k}}^{z} & =G_{a} \cos \left(\vec{k}^{\prime}+\vec{k}\right) \cdot \vec{R} / 2,
\end{aligned}
$$

mostrando que nosso Hamiltoniano respeita a forma geral de interação entre elétrons de condução e um sistema de dois níveis.

\section{4 - Hamiltoniano Completo}

Utilizando as Eqs. (2.2), (2.3), (2.17) e (2.19) o Hamiltoniano completo, Eq.(2.1), pode ser escrito como

$$
\begin{aligned}
& H=\int d \vec{k} \varepsilon_{\vec{k}} c_{\vec{k}}^{+} c_{\vec{k}}-\frac{\Delta}{2}\left(d_{1}^{+} d_{2}+d_{2}^{+} d_{1}\right)+ \\
& +G \Psi^{+}(\vec{R} / 2) \Psi(\vec{R} / 2) d_{1}^{+} d_{1}+G \Psi^{+}(-\vec{R} / 2) \Psi(-\vec{R} / 2) d_{2}^{+} d_{2}+ \\
& +G_{a} \Psi^{+}(\vec{R} / 2) \Psi(-\vec{R} / 2) d_{2}^{+} d_{1}+G_{a} \Psi^{+}(-\vec{R} / 2) \Psi(\vec{R} / 2) d_{1}^{+} d_{2}
\end{aligned}
$$

Esse Hamiltoniano é interpretado, então, como o de um íon tunelando entre dois mínimos locais de potencial localizados no sítio $1(\vec{R} / 2)$ e no sítio $2(-\vec{R} / 2)$. $\mathrm{O}$ íon ao tunelar espalha os elétrons da $\mathrm{BC}$ por meio do potencial de Coulomb, que tem magnitude constante mas cujo centro varia como resultado do "pulo" do íon. O tunelamento ocorre por dois motivos: tunelamento direto e tunelamento assistido.

Necessitamos, agora, diagonalizar o Hamiltoniano acima para obter os autovalores do sistema e calcular o calor específico. Para isso, utilizamos o GRN, técnica numérica que exige várias adaptações do Hamiltoniano para que possa ser implementado computacionalmente. Mostraremos como isso é feito no próximo capítulo. 


\section{Capítulo 3}

\section{Grupo de Renormalização Numérico}

Neste capítulo faremos a adaptação do Hamiltoniano (2.25) ao GRN. A técinca é basicamente aquela proposta por Wilson [14], porém adaptada ao problema de dois centros seguindo os trabalhos das Refs.[17,20] . Essa técnica consiste em aproximar o contínuo da BC por um conjunto discreto de estados. Projetado nessa base discreta, o Hamiltoniano do modelo é diagonalizado por um processo iterativo, e a partir dos autovalores obtidos calcula-se o calor específico.

A conversão do contínuo da $\mathrm{BC}$ a uma base que possa ser tratada numericamente é feita em dois passos. No primeiro, a banda é discretizada, porém o conjunto de estados discretos gerado no processo é infinito. No segundo passo os estados são truncados, gerando uma base finita.

Antes desses passos fundamentais do GRN é conveniente, como veremos, mudar os operadores de nosso Hamiltoniano, que estão em função do momento, para operadores de energia.

\section{1 - Adaptação do Hamiltoniano de dois Centros ao GRN}

Como já dissemos, consideramos a BC do metal simétrica em relação ao nível de energia de Fermi $\left(\varepsilon_{F}\right)$ com largura $2 D$ e isotrópica, isto é, a energia $\varepsilon_{\vec{k}}$ depende apenas do módulo do momento $\vec{k}$ :

$$
\varepsilon_{\vec{k}}=\frac{\hbar^{2} k^{2}}{2 m}=\varepsilon_{k},
$$


sendo $m$ a massa do elétron.

Assim, podemos reescrever o Hamiltoniano da Eq. (2.25) em função apenas da energia. Devemos, para isso, transformar os operadores fermiônicos de momento para energia. A seguir faremos essa transformação.

\subsection{1 - Transformação do Operador de Campo}

Podemos reescrever o operador de campo da seguinte maneira:

$$
\begin{aligned}
& \Psi(\vec{R} / 2)=\int d^{3} k e^{i \vec{k} \cdot \frac{\vec{R}}{2}} c_{\vec{k}}=\int d^{3} k \int d \varepsilon \delta\left(\varepsilon-\varepsilon_{k}\right) e^{i \vec{k} \cdot \frac{\vec{R}}{2}} c_{\vec{k}} \equiv \\
& \equiv \int_{-D}^{D} d \varepsilon \sqrt{\rho(\varepsilon)} c_{1 \varepsilon}
\end{aligned}
$$

no qual definimos

$$
c_{1 \varepsilon}=\frac{1}{\sqrt{\rho(\varepsilon)}} \int d^{3} k e^{i \vec{k} \cdot \frac{\vec{R}}{2}} \delta\left(\varepsilon-\varepsilon_{k}\right) c_{\vec{k}}
$$

e

$$
\rho(\varepsilon)=\int d^{3} k \delta\left(\varepsilon-\varepsilon_{k}\right)=4 \pi k^{2}(\varepsilon) \frac{d k}{d \varepsilon_{k}}
$$

é a densidade de estados $\rho(\varepsilon)$ que aparece devido à normalização do operador $c_{1 \varepsilon}$. Este operador destrói elétrons da $\mathrm{BC}$ com estados de energia $\varepsilon$ e localizados em $\vec{R} / 2$. Analogamente, temos

$$
\Psi(-\vec{R} / 2)=\int_{-D}^{D} d \varepsilon \sqrt{\rho(\varepsilon)} c_{2 \varepsilon}
$$

sendo 


$$
c_{2 \varepsilon}=\frac{1}{\sqrt{\rho(\varepsilon)}} \int d^{3} k e^{-i \vec{k} \cdot \vec{R} / 2} \delta\left(\varepsilon-\varepsilon_{k}\right) c_{k}
$$

Esses novos operadores obedecem individualmente as relações de anticomutação convencionais

$$
\left\{c_{1 \varepsilon}, c_{1 \varepsilon^{+}}^{+}\right\}=\left\{c_{2 \varepsilon}, c_{2 \varepsilon^{\prime}}^{+}\right\}=\delta\left(\varepsilon-\varepsilon^{\prime}\right)
$$

Contudo, eles não são ortogonais, ou seja:

$$
\left\{c_{1 \varepsilon}, c_{2 \varepsilon^{\prime}}^{+}\right\}=\delta\left(\varepsilon-\varepsilon^{\prime}\right) \frac{\operatorname{sen}[k(\varepsilon) R]}{k(\varepsilon) R}
$$

Vamos, então, construir novos operadores que sejam ortogonais. Para isso, vamos aproveitar a simetria de inversão do nosso modelo, ou seja trocar o índice 1 por 2 não altera o problema, e escolher novos operadores com paridades bem definidas. Assim, definimos os operadores

$$
s_{\varepsilon+}=\frac{1}{A_{+}}\left(\frac{c_{1 \varepsilon}+c_{2 \varepsilon}}{2}\right) \quad \text { e } \quad s_{\varepsilon-}=\frac{1}{A_{-}}\left(\frac{c_{1 \varepsilon}-c_{2 \varepsilon}}{2}\right)
$$

com

$$
A_{ \pm}^{2}=\frac{1}{2}\left(1 \pm \frac{\sin k(\varepsilon) R}{k(\varepsilon) R}\right)
$$

sendo $s_{\varepsilon+}$ par e $s_{\varepsilon-}$ ímpar. Dessa forma, as relações de anticomutação ficam satisfeitas, ou seja:

$$
\left\{s_{\varepsilon+}, s_{\varepsilon^{\prime}-}\right\}=\left\{s_{\varepsilon+}, s_{\varepsilon^{\prime}-}^{+}\right\}=0 \text { e }\left\{s_{\varepsilon \pm}, s_{\varepsilon^{\prime} \pm}^{+}\right\}=\delta\left(\varepsilon-\varepsilon^{\prime}\right)
$$


Reescrevendo os operadores $c_{1 \varepsilon}$ e $c_{2 \varepsilon}$ em função de $s_{\mathcal{E}_{+}}$e $s_{\mathcal{E}^{-}}$, e substituindo-os nos operadores de campo, obtemos:

$$
\Psi( \pm \vec{R} / 2)=\int_{-D}^{D} d \varepsilon \sqrt{\rho(\varepsilon)}\left(A_{+} s_{\varepsilon+} \pm A_{-} s_{\varepsilon_{-}}\right)
$$

\subsection{2 - Transformação dos Termos de Espalhamento e Tunelamento Assistido}

Podemos, portanto, com os operadores de campo escritos acima, reescrever facilmente o termo de espalhamento, Eq. (2.17), como

$$
\begin{aligned}
H_{e s p} & =G \iint_{-D}^{D} d \varepsilon d \varepsilon^{\prime} \sqrt{\rho(\varepsilon) \rho\left(\varepsilon^{\prime}\right)}\left(A_{+}^{2} s_{\varepsilon+}^{+} s_{\varepsilon^{\prime}+}+A_{-}^{2} s_{\varepsilon-}^{+} s_{\varepsilon^{\prime}-}+\right. \\
& \left.+A_{+} A_{-} s_{\varepsilon+}^{+} s_{\varepsilon^{\prime}-}+A_{-} A_{+} s_{\varepsilon-}^{+} s_{\varepsilon^{\prime}+}\right) d_{1}^{+} d_{1}+ \\
& +G \iint_{-D}^{D} d \varepsilon d \varepsilon^{\prime} \sqrt{\rho(\varepsilon) \rho\left(\varepsilon^{\prime}\right)}\left(A_{+}^{2} s_{\varepsilon+}^{+} s_{\varepsilon^{\prime}+}+A_{-}^{2} s_{\varepsilon_{-}}^{+} s_{\varepsilon^{\prime}-}-\right. \\
& \left.-A_{+} A_{-} s_{\varepsilon+}^{+} s_{\varepsilon^{\prime}-}-A_{-} A_{+} s_{\varepsilon-}^{+} s_{\varepsilon^{\prime}+}\right) d_{2}^{+} d_{2},
\end{aligned}
$$

e para o tunelamento assistido, Eq. (2.19), temos

$$
\begin{aligned}
H_{a s s} & =G_{a} \iint_{-D}^{D} d \varepsilon d \varepsilon^{\prime} \sqrt{\rho(\varepsilon) \rho\left(\varepsilon^{\prime}\right)}\left(A_{+}^{2} s_{\varepsilon_{+}+}^{+} s_{\varepsilon^{\prime}+}-A_{-}^{2} s_{\varepsilon-}^{+} s_{\varepsilon^{\prime}-}+\right. \\
& \left.+A_{+} A_{-} s_{\varepsilon_{+}+}^{+} s_{\varepsilon^{\prime}-}-A_{-} A_{+} s_{\varepsilon-}^{+} s_{\varepsilon^{\prime}+}\right) d_{1}^{+} d_{2}+ \\
& +G_{a} \iint_{-D}^{D} d \varepsilon d \varepsilon^{\prime} \sqrt{\rho(\varepsilon) \rho\left(\varepsilon^{\prime}\right)\left(A_{+}^{2} s_{\varepsilon+}^{+} s_{\varepsilon^{\prime}+}-A_{-}^{2} s_{\varepsilon-}^{+} s_{\varepsilon^{\prime}-}-\right.} \\
& \left.-A_{+} A_{-} s_{\varepsilon+}^{+} s_{\varepsilon^{\prime}-}+A_{-} A_{+} s_{\varepsilon-}^{+} s_{\varepsilon^{\prime}+}\right) d_{2}^{+} d_{1} .
\end{aligned}
$$

Tendo escrito $H_{\text {esp }}$ e $H_{\text {ass }}$ em função dos operadores de energia, nosso próximo passo será reescrever a $\mathrm{BC}$ em função desses operadores. A próxima seção será dedicada a esse fim. 


\subsection{3 - Transformação da Banda de Condução}

Agora iremos representar $H_{b c}$ em termos dos novos operadores, $s_{\varepsilon_{+}}$e $s_{\varepsilon_{-}-}$. O primeiro passo será expandir os operadores $c_{\vec{k}}$ em harmônicos esféricos

$$
c_{\vec{k}}=\frac{1}{k} \sum_{l m} Y_{l m} c_{k l m}
$$

onde $k$ é o módulo do momento $\vec{k}$, que aparece devido à normalização de $c_{\vec{k}}$. $\mathrm{O}$ eixo $z$ é tomado ao longo da linha que une os sítios.

Assim,

$$
H_{b c}=\int d^{3} k \varepsilon_{\vec{k}} c_{\vec{k}}^{+} c_{\vec{k}}=\sum_{l m} \int d k \varepsilon_{k} c_{k l m}^{+} c_{k l m}
$$

onde utilizamos a condição de ortonormalidade dos harmônicos esféricos, isto é, $\int d \Omega Y_{l m}^{*}(\theta, \varphi) Y_{l, m},(\theta, \varphi)=\delta_{l l}, \delta_{m m}$

Apenas os elétrons com $m=0$ precisam ser considerados em $H_{b c}$. Isso porque os demais não se acoplam aos estados $d_{1}$ e $d_{2}$, visto que temos simetria cilíndrica em torno do eixo $z$.

Levando em conta esse fato e a isotropia da banda de condução, escrevemos

$$
c_{\vec{k}}=\frac{1}{k} \sum_{l} Y_{l 0} c_{k l}
$$

sendo $c_{k l} \equiv c_{k l 0}$. Assim,

$$
H_{b c}=\int d^{3} k \varepsilon_{\vec{k}} c_{\vec{k}}^{+} c_{\vec{k}}=\sum_{l} \int d k \varepsilon_{k} c_{k l}^{+} c_{k l}
$$

Novamente, definiremos operadores fermiônicos depedentes apenas da energia $\varepsilon$ para substituir os operadores $c_{k l}$ : 


\subsection{3 - Transformação da Banda de Condução}

Agora iremos representar $H_{b c}$ em termos dos novos operadores, $s_{\varepsilon+}$ e $s_{\varepsilon_{-}-}$. O primeiro passo será expandir os operadores $c_{\vec{k}}$ em harmônicos esféricos

$$
c_{\vec{k}}=\frac{1}{k} \sum_{l m} Y_{l m} c_{k l m}
$$

onde $k$ é o módulo do momento $\vec{k}$, que aparece devido à normalização de $c_{\vec{k}}$. O eixo $z$ é tomado ao longo da linha que une os sítios.

Assim,

$$
H_{b c}=\int d^{3} k \varepsilon_{\vec{k}} c_{\vec{k}}^{+} c_{\vec{k}}=\sum_{l m} \int d k \varepsilon_{k} c_{k l m}^{+} c_{k l m}
$$

onde utilizamos a condição de ortonormalidade dos harmônicos esféricos, isto é, $\int d \Omega Y_{l m}^{*}(\theta, \varphi) Y_{l^{\prime} m^{\prime}}(\theta, \varphi)=\delta_{l l^{\prime}} \delta_{m m^{\prime}}$.

Apenas os elétrons com $m=0$ precisam ser considerados em $H_{b c}$. Isso porque os demais não se acoplam aos estados $d_{1}$ e $d_{2}$, visto que temos simetria cilíndrica em torno do eixo $z$.

Levando em conta esse fato e a isotropia da banda de condução, escrevemos

$$
c_{\vec{k}}=\frac{1}{k} \sum_{l} Y_{l 0} c_{k l}
$$

sendo $c_{k l} \equiv c_{k l 0}$. Assim,

$$
H_{b c}=\int d^{3} k \varepsilon_{\vec{k}} c_{\vec{k}}^{+} c_{\vec{k}}=\sum_{l} \int d k \varepsilon_{k} c_{k l}^{+} c_{k l}
$$

Novamente, definiremos operadores fermiônicos depedentes apenas da energia $\varepsilon$ para substituir os operadores $c_{k l}$ :

$$
s_{\varepsilon \pm}=\frac{1}{A_{ \pm}} \sum_{l_{ \pm}}^{\infty} i^{l} \sqrt{2 l+1} j_{l}\left(\frac{k R}{2}\right) c_{d}
$$

sendo $l_{+}=0,2,4, \ldots$ e $l_{-}=1,3,5, \ldots$ Essas equações são da forma 


$$
c_{k l}=\sqrt{\frac{d \varepsilon}{d k}} c_{d}
$$

$\mathrm{O}$ fator $\sqrt{d \varepsilon / d k}$ aparece para normalizar $c_{\varepsilon l}$ de forma que a relação de anticomutação $\left\{c_{k l}, c_{k^{\prime} l}\right\}=\delta_{k k}, \delta_{l l}$, é obedecida. Assim,

$$
H_{b c}=\sum_{l} \int_{-D}^{D} \varepsilon d \varepsilon c_{\varepsilon l}^{+} c_{\varepsilon l}
$$

Para que possamos expressar o operador $c_{\varepsilon l}$ em função de $s_{\varepsilon+}$ e $s_{\varepsilon-}$, devemos voltar às expressões de $c_{1 \varepsilon}$ e $c_{2 \varepsilon}$, Eqs. (3.3) e (3.6), e reescrevê-las da seguinte maneira:

$$
c_{1 \varepsilon}=\sum_{l=0}^{\infty} i \sqrt{2 l+1} j_{l}\left(\frac{k R}{2}\right) c_{\varepsilon l} \quad \text { e } \quad c_{2 \varepsilon}=\sum_{l=0}^{\infty}(-i)^{l} \sqrt{2 l+1} j_{l}\left(\frac{k R}{2}\right) c_{\varepsilon l},
$$

onde utilizamos o fato que as funções de onda plana da forma $e^{i \vec{k} \cdot \vec{r}}$ podem ser expandidas em termos das funções de Bessel $j_{l}(k r)$ e dos harmônicos esféricos $Y_{l 0}$,

$$
e^{i \vec{k} \cdot \frac{\vec{R}}{2}}=\sum_{l=0}^{\infty} i^{l} \sqrt{4 \pi(2 l+1)} j_{l}\left(\frac{k R}{2}\right) Y_{l 0}
$$

Substituindo, agora, as relações (3.21) na definição de $s_{\varepsilon^{+}}$e $s_{\mathcal{\varepsilon}^{-}}$, Eq. (3.9), obtemos:

$$
s_{\varepsilon \pm}=\frac{1}{A_{ \pm}} \sum_{l_{ \pm}}^{\infty} i^{l} \sqrt{2 l+1} j_{l}\left(\frac{k R}{2}\right) c_{d}
$$

sendo $l_{+}=0,2,4, \ldots$ e $l_{-}=1,3,5, \ldots$ Essas equações são da forma 


$$
s_{\varepsilon p}=\sum_{l} u_{p l} c_{\varepsilon l}
$$

sendo

$$
u_{p l}=\frac{1}{A_{+}} i^{l} \sqrt{2 l+1} j_{l}\left(\frac{k R}{2}\right) \delta_{1,(-1)^{l}} ; \quad \text { se } p=+
$$

ou

$$
u_{p l}=\frac{1}{A_{-}} i^{l} \sqrt{2 l+1} j_{l}\left(\frac{k R}{2}\right) \delta_{-1,(-1)^{l}} ; \quad \text { se } p=-
$$

Esses coeficientes obedecem à relação de ortogonalidade

$$
\sum_{l} u_{p l} u_{p, l}^{*}=\delta_{p p^{\prime}}
$$

por isso, podemos fazer uma transformação ortonormal na Eq. (3.24), obtendo assim o operador $c_{\varepsilon l}$ em termos de $s_{\varepsilon p}$ :

$$
c_{\varepsilon l}=\sum_{p} u_{p l}^{*} s_{\varepsilon p}
$$

Observando que $H_{\text {esp }}$ e $H_{\text {ass }}$ são definidos por operadores com paridade definida, isto é, os elétrons com funções de onda sem paridade não se acoplam com o íon tunelante (eles não influenciam no cálculo de propriedades termodinâmicas do ín), podemos desconsiderar os termos em $H_{b c}$ que não têm paridade. Assim, em termos dos operadores $s_{\varepsilon p}, H_{b c}$ se escreve como

$$
\begin{gathered}
H_{b c}=\sum_{p p^{\prime} l} \int_{-D}^{D} d \varepsilon \varepsilon u_{p l} u_{p^{\prime} l}^{*} s_{\varepsilon p}^{+} s_{\varepsilon p}=\sum_{p} \int_{-D}^{D} d \varepsilon \varepsilon s_{\varepsilon p}^{+} s_{\varepsilon p} \\
H_{b c}=\int_{-D}^{D} d \varepsilon \varepsilon\left(s_{\varepsilon+}^{+} s_{\varepsilon^{+}}+s_{\varepsilon-}^{+} s_{\varepsilon^{-}}\right) .
\end{gathered}
$$




\subsection{4 - Hamiltoniano Completo}

Escrevendo o Hamiltoniano completo em termos dos novos operadores temos

$$
\begin{aligned}
H & =\int_{-D}^{D} d \varepsilon \varepsilon\left(s_{\varepsilon+}^{+} s_{\varepsilon^{+}}+s_{\varepsilon^{-}}^{+} s_{\varepsilon^{-}}\right)-\frac{\Delta}{2}\left(d_{1}^{+} d_{2}+d_{2}^{+} d_{1}\right)+ \\
& +G \iint_{-D}^{D} d \varepsilon d \varepsilon^{\prime} \sqrt{\rho(\varepsilon) \rho\left(\varepsilon^{\prime}\right)}\left[A_{+}^{2} s_{\varepsilon^{+}+}^{+} s_{\varepsilon^{\prime}+}+A_{-}^{2} s_{\varepsilon^{-}}^{+} s_{\varepsilon^{\prime}-}\right]\left[d_{1}^{+} d_{1}+d_{2}^{+} d_{2}\right]+ \\
& +G \iint_{-D}^{D} d \varepsilon d \varepsilon^{\prime} \sqrt{\rho(\varepsilon) \rho\left(\varepsilon^{\prime}\right)} A_{+} A_{-}\left[s_{\varepsilon^{+}+}^{+} s_{\varepsilon^{\prime}-}+s_{\varepsilon^{-}}^{+} s_{\varepsilon^{\prime}+}\right]\left[d_{1}^{+} d_{1}-d_{2}^{+} d_{2}\right]+ \\
& +G_{a} \iint_{-D}^{D} d \varepsilon d \varepsilon^{\prime} \sqrt{\rho(\varepsilon) \rho\left(\varepsilon^{\prime}\right)}\left[A_{+}^{2} s_{\varepsilon^{+}}^{+} s_{\varepsilon^{\prime}+}-A_{-}^{2} s_{\varepsilon^{-}}^{+} s_{\varepsilon^{\prime}-}\right]\left[d_{1}^{+} d_{2}+d_{2}^{+} d_{1}\right]+ \\
& +G_{a} \iint_{-D}^{D} d \varepsilon d \varepsilon^{\prime} \sqrt{\rho(\varepsilon) \rho\left(\varepsilon^{\prime}\right)} A_{+} A_{-}\left[s_{\varepsilon^{+}}^{+} s_{\varepsilon^{\prime}+}-s_{\varepsilon^{+}+} s_{\varepsilon^{\prime}-}\right]\left[d_{2}^{+} d_{1}-d_{1}^{+} d_{2}\right]
\end{aligned}
$$

Nesse Hamiltoniano, os acoplamentos $\rho(\varepsilon)$ e $A_{ \pm}$do íon com os operadores $s_{\varepsilon p}$ dependem da energia $\varepsilon$. Isso gera dificuldades para a implementação do GRN. Porém, como estamos interessados em propriedades termodinâmicas a baixas temperaturas $\left(k_{B} T<<D\right)$, podemos considerar os acoplamentos constantes e iguais aos seus valores no nível de Fermi. Tal procedimento é adotado com sucesso no caso de uma impureza [15]. Também foi utilizado no caso do modelo de Kondo de Tunelamento de duas impurezas sem o tunelamento assistido [17,21], sendo que neste caso, resultados preliminares do calor específico mostram que não há alteração no resultado, fazendo-se ou não os acoplamentos constantes. Já no caso do modelo de Kondo de duas impurezas magnéticas essa aproximação altera a simetria partículaburaco do modelo o que causa resultados espúrios [22,23]. O nosso modelo não possui simetria partícula-buraco, por isso assumiremos válida essa aproximação. Contudo, já existe técnica para implementação do GRN sem fazer tal aproximação [24], a qual não pudemos implementar nesse trabalho devido ao tempo limitado que tivemos.

Assim, para $k_{B} T \ll<$, utilizamos

$$
\begin{aligned}
& A_{ \pm}(\varepsilon)=A_{ \pm}\left(\varepsilon_{F}\right) \\
& \rho(\varepsilon)=\int d^{3} k \delta\left(\varepsilon-\varepsilon_{k}\right)=\rho\left(\varepsilon_{F}\right)=\frac{1}{D}
\end{aligned}
$$


sendo esta última consistente com a normalização dos operadores de campo, Eqs. (3.2) e (3.5).

Com essas aproximações e dimensionalizando em termos de $D$, para uma melhor praticidade computacional, obtemos

$$
\begin{aligned}
H & =\int_{-1}^{1} d \varepsilon \varepsilon\left(s_{\varepsilon+}^{+} s_{\varepsilon^{+}}+s_{\varepsilon^{-}}^{+} s_{\varepsilon^{-}}\right)-\frac{\Delta}{2}\left(d_{1}^{+} d_{2}+d_{2}^{+} d_{1}\right)+ \\
& +G \iint_{-1}^{1} d \varepsilon d \varepsilon^{\prime}\left[A_{+}^{2} s_{\varepsilon+}^{+} s_{\varepsilon^{\prime}+}+A_{-}^{2} s_{\varepsilon_{-}^{+}}^{+} s_{\varepsilon^{\prime}-}\right]\left[d_{1}^{+} d_{1}+d_{2}^{+} d_{2}\right]+ \\
& +G \iint_{-1}^{1} d \varepsilon d \varepsilon^{\prime} A_{+} A_{-}\left[s_{\varepsilon^{+}}^{+} s_{\varepsilon^{\prime}-}+s_{\varepsilon_{-}}^{+} s_{\varepsilon^{\prime}+}\right]\left[d_{1}^{+} d_{1}-d_{2}^{+} d_{2}\right]+ \\
& +G_{a} \iint_{-1}^{1} d \delta d \varepsilon^{\prime}\left[A_{+}^{2} s_{\varepsilon^{+}}^{+} s_{\varepsilon^{\prime}+}-A_{-}^{2} s_{\varepsilon^{-}}^{+} s_{\varepsilon^{\prime}-}\right]\left[d_{1}^{+} d_{2}+d_{2}^{+} d_{1}\right]+ \\
& +G_{a} \iint_{-1}^{1} d \delta d \varepsilon^{\prime} A_{+} A_{-}\left[s_{\varepsilon^{-}}^{+} s_{\varepsilon^{\prime}+}-s_{\varepsilon^{+}+}^{+} s_{\varepsilon^{\prime}-}\right]\left[d_{2}^{+} d_{1}-d_{1}^{+} d_{2}\right]
\end{aligned}
$$

no qual fizemos a seguinte redefinição

$$
\frac{H}{D} \rightarrow H, \quad \frac{\varepsilon}{D} \rightarrow \varepsilon, \quad \frac{\Delta}{D} \rightarrow \Delta, \quad \frac{G}{D} \rightarrow G, \quad \frac{G_{a}}{D} \rightarrow G_{a}
$$

que torna $H$ adimensional; a nomenclatura foi mantida a mesma por conveniência.

Todas essas transformações que fizemos até agora foram para trabalhar com operadores de energia ao invés de momento, aproveitando a isotropia da banda de condução. Porém, o Hamiltoniano que obtemos ainda atua no contínuo de energia da banda, o que impede a implementação computacional. Nesse ponto surge um passo fundamental dentro da técnica do GRN que é dividir a banda em diversos intervalos através da discretização logarítmica, da qual falaremos a seguir.

\section{2 - Discretização Logarítmica}

Como estamos interessados em propriedades do sistema em baixas temperaturas ( $K_{B} T \ll D$ ), vamos usar um método de discretização da banda que enfatize a região de baixa energia. A BC do nosso modelo será discretizada de modo semelhante ao de Wilson [14], sendo que o contínuo de energia será substituído por 
um conjunto de pontos dispostos num intervalo de escalas de energia logarítmica, conforme mostra a Fig. 3.1.

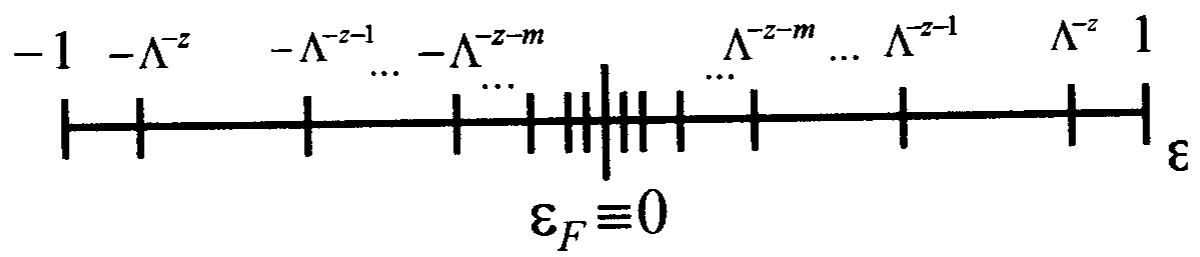

Figura 3.1 - Banda de condução discretizada logaritmicamente. As energias são medidas em relação ao nível de Fermi , $\varepsilon_{F}$, em unidades de meia largura, $D$, da banda.

No procedimento original não havia o parâmetro $z$, apenas $\Lambda(\operatorname{com} \Lambda>1)$, que é a razão entre duas energias discretas consecutivas (quando $\Lambda=1$ o limite contínuo é recuperado e o procedimento torna-se exato). $\mathrm{O}$ parâmetro $z$ generaliza a discretização logarítmica, e pode assumir qualquer valor no intervalo $0<z \leq 1$. Sua introdução foi feita por Yoshida et. al. [16] e possibilitou usar o GRN para calcular propriedades dinâmicas, como densidade espectral, sendo aplicado com sucesso a vários modelos $[18,20]$. Posteriormente, descobriu-se que ele era capaz de eliminar oscilações originadas do método numérico nas curvas de propriedades termodinâmicas $[17,25,26]$, característica limitante do método tradicional.

Como exemplo, a Fig. 3.2 mostra a contribuição do íon para o calor específico do metal, $C_{i o n}$, em função de $k_{B} T / D$. A curva azul foi obtida utilizando o GRN padrão $(z=1)$. Nela podemos observar as oscilações originadas da discretização, sendo que sua amplitude depende de $\Lambda$ através do fator $\exp \left(-\pi^{2} / \ln \Lambda\right)$ [26], ou seja, quanto menor for $\Lambda$ menor a amplitude de oscilação. Porém, à medida que diminuimos o valor de $\Lambda$ o tempo computacional gasto aumenta muito.

Valter Líbero e J. Vítor [17] mostraram que a média sobre valores de $z$ suaviza a curva de calor específico para o problema de uma partícula tunelante sem o termo assistido.

Seguindo o mesmo procedimento, podemos suavizar a curva de calor específico da seguinte maneira 


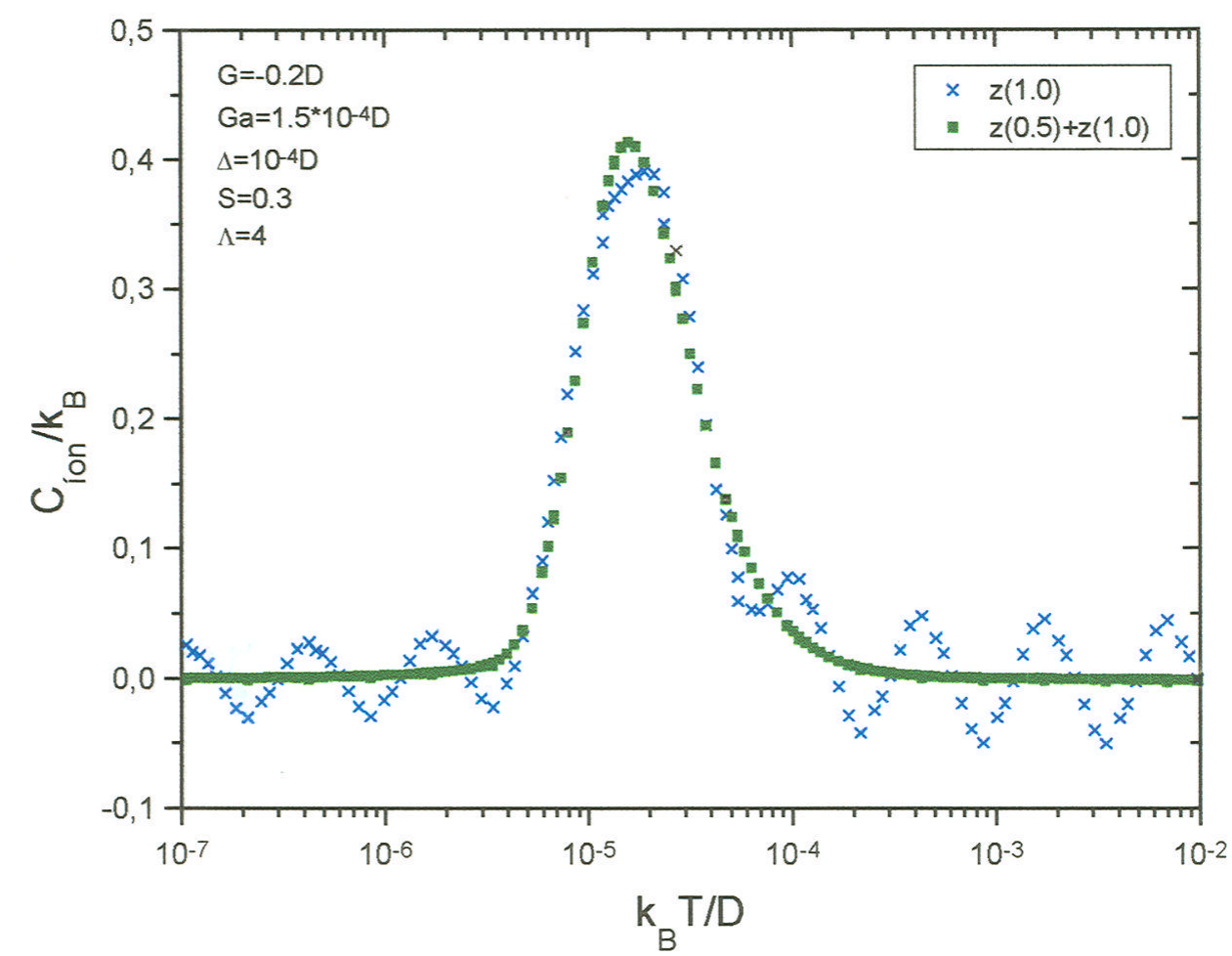

Figura 3.2: Curvas de calor específico mostrando o efeito do procedimento intercalado. Pode-se ver que a utilização de dois z's suaviza a curva.

$$
C_{\text {ion }}(T)=\int_{0}^{1} C_{\text {ion }}(T, z) d z
$$

O que se observa na prática é que, para a maior parte das curvas mostradas nesta dissertação, apenas dois valores de $z$ que diferem por 0.5 são suficientes para suavizar curvas obtidas com $\Lambda=4$. Portanto, podemos suavizar curvas de $C_{\text {ion }}$, simplesmente fazendo [26]

$$
C_{\text {ion }}(T)=\frac{C_{\text {ion }}(T, z=0.5)+C_{i o n}(T, z=1.0)}{2}
$$

Esse é o chamado procedimento intercalado. Com ele, podemos eliminar as oscilações sem usar $\Lambda$ muito pequeno, como mostra a curva verde da Fig. 3.2.

As curvas de $C_{i o n}$ mostradas aqui servem apenas para ilustrar a utilização do parâmentro $z$. No capítulo 4 nos aprofundaremos na interpretação dessas curvas. 
Voltando à discretização logarítmica, ela preserva uma característica importante da $\mathrm{BC}$ que é a invariância por transformações de escala de energia próximo ao nível de Fermi (Fig. 3.3). Contudo, a invariância ocorre agora por múltiplos de $\Lambda$, conforme mostra a Fig. 3.1.

Com a banda discretizada, temos que o $m$-ésimo intervalo é aquele compreendido entre $\Lambda^{-z-m-1}$ e $\Lambda^{-z-m}(m=0,1,2, \ldots)$, com exceção do topo da banda. Definiremos um conjunto completo de funções ortonormais $\Phi_{m t}$, para cada um desses intervalos:

$$
\Phi_{t}^{ \pm}(\varepsilon)= \begin{cases}\frac{1}{\left(1-\Lambda^{-z}\right)^{1 / 2}} \exp \left( \pm \frac{2 \pi i}{1-\Lambda^{-z}} t \varepsilon\right), & \text { se } \Lambda^{-z}< \pm \varepsilon<1 \\ 0, & \text { fora do intervalo }\end{cases}
$$

$$
\Phi_{m t}^{ \pm}(\varepsilon)= \begin{cases}\frac{\Lambda^{(m+z) / 2}}{\left(1-\Lambda^{-1}\right)^{1 / 2}} \exp \left( \pm \frac{2 \pi i \Lambda^{m+z}}{1-\Lambda^{-1}} t \varepsilon\right), & \text { se } \Lambda^{-(z+m+1)}< \pm \varepsilon<\Lambda^{-(z+m)} \\ 0, & \text { fora do intervalo }\end{cases}
$$

onde $t$ é $o$ índice da série de Fourier $(-\infty<t<\infty, t$ inteiro $)$ e $+(-)$ indica a definição da função para $\varepsilon$ positivo (negativo). Usamos também a condição da função ter o mesmo valor nos limites dos intervalos.

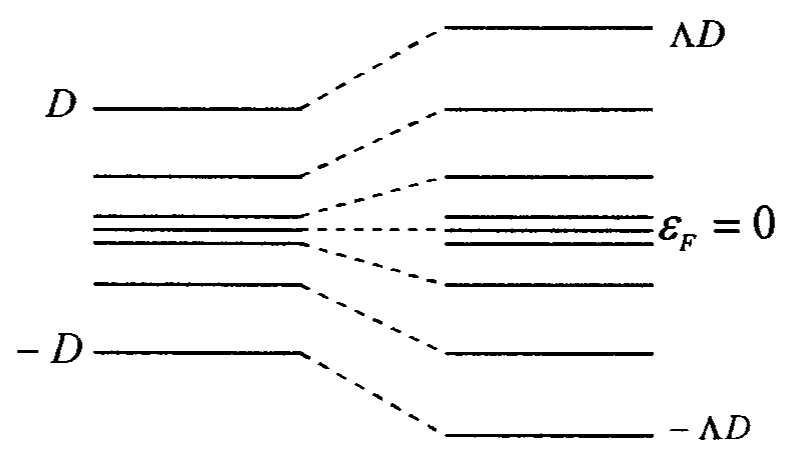

Figura 3.3- Banda de condução representada por alguns de seus níveis (centrada no nível de Fermi e com largura 2D). Do lado equerdo ela está em escala 'normal', do lado direito a escala foi multiplicada por um fator $\Lambda$. Observe que a estrutura de níveis perto do nível de Fermi se mantém constante. 
A expansão dos operadores $S_{\varepsilon p}(\mathrm{p}=+,-)$ nessa base,

$$
s_{\varepsilon p}=\sum_{m, t}\left(a_{p t} \Phi_{t}^{+}+b_{p t} \Phi_{t}^{-}+a_{p m t} \Phi_{m t}^{+}+b_{p m t} \Phi_{m t}^{-}\right)
$$

define os seguintes operadores fermiônicos e discretos

$$
\begin{array}{ll}
a_{p t}=\int_{-1}^{1} d \varepsilon\left[\Phi_{t}^{+}(\varepsilon)\right]^{*} s_{\varepsilon p}, & b_{p t}=\int_{-1}^{1} d \varepsilon\left[\Phi_{t}^{-}(\varepsilon)\right]^{*} s_{\varepsilon p} \\
a_{p m t}=\int_{-1}^{1} d \varepsilon\left[\Phi_{m t}^{+}(\varepsilon)\right]^{*} s_{\varepsilon p}, & b_{p m t}=\int_{-1}^{1} d \varepsilon\left[\Phi_{m t}^{-}(\varepsilon)\right]^{*} s_{\varepsilon p}
\end{array}
$$

Se voltarmos à Eq. (3.33) perceberemos que o íon interage diretamente com a $\mathrm{BC}$ apenas através do termo $\int d \varepsilon s_{\varepsilon p}$. Assim, utilizando as Eqs. (3.37), (3.38) e (3.39), encontramos que

$$
\int_{-1}^{1} d \varepsilon s_{\varepsilon p}=\left(1-\Lambda^{-z}\right)^{1 / 2}\left(a_{p 0}+b_{p 0}\right)+\left(1-\Lambda^{-1}\right)^{1 / 2} \sum_{m} \Lambda^{\frac{-(m+z)}{2}}\left(a_{p m 0}+b_{p m 0}\right)
$$

ou seja, o ín interage diretamente apenas com operadores com $t=0$. Assim, podemos expressar $H_{b c}$, Eq.(3.30), com os operadores da Eq. (3.41), mantendo somente os termos com $t=0$. E ainda, sabendo que cada função $\Phi(\varepsilon)$ é nula fora de seu respectivo intervalo, podemos escrever $H_{b c}$ como

$$
H_{b c}=\sum_{p} \frac{\left(1+\Lambda^{-z}\right)}{2}\left(a_{p}^{+} a_{p}-b_{p}^{+} b_{p}\right)+\sum_{p m} \frac{\Lambda^{-(z+m)}\left(1+\Lambda^{-1}\right)}{2}\left(a_{p m}^{+} a_{p m}-b_{p m}^{+} b_{p m}\right)
$$

onde omitimos o índice $t=0$ nos operadores.

Usando a Eq. (3.41) podemos definir um novo operador $f_{0 p}$ dado por

$$
f_{0 p}=\frac{1}{\sqrt{2}} \int_{-1}^{1} d \varepsilon s_{\varepsilon p}
$$


onde $p=+,-. O$ fator $1 / \sqrt{2}$ na Eq. (3.43) é para normalizar $f_{0 p}$ tal que a relação de anticomutação $\left\{f_{0 p}, f_{0 p^{\prime}}^{+}\right\}=\delta_{p p^{\prime}}$ seja satisfeita. Esses operadores são os únicos que se acoplam diretamente com o íon. Ou seja, apenas estados eletrônicos com paridade definida se acoplam com ele. $O$ nosso Hamiltoniano é escrito, então, como

$$
\begin{aligned}
H= & \sum_{p} \frac{\left(1+\Lambda^{-z}\right)}{2}\left(a_{p}^{+} a_{p}-b_{p}^{+} b_{p}\right)+\sum_{p m} \frac{\Lambda^{-(z+m)}\left(1+\Lambda^{-1}\right)}{2}\left(a_{p m}^{+} a_{p m}-b_{p m}^{+} b_{p m}\right)+ \\
& +2 G\left[A_{+}^{2} f_{0+}^{+} f_{0+}+A_{-}^{2} f_{0-}^{+} f_{0-}\right]\left[d_{1}^{+} d_{1}+d_{2}^{+} d_{2}\right]+ \\
& +2 G A_{+} A_{-}\left[f_{0+}^{+} f_{0-}+f_{0-}^{+} f_{0+}\right]\left[d_{1}^{+} d_{1}-d_{2}^{+} d_{2}\right]+ \\
& +2 G_{a}\left[A_{+}^{2} f_{0+}^{+} f_{0+}-A_{-}^{2} f_{0-}^{+} f_{0-}\right]\left[d_{2}^{+} d_{1}+d_{1}^{+} d_{2}\right]+ \\
& +2 G_{a} A_{+} A_{-}\left[f_{0-}^{+} f_{0+}-f_{0+}^{+} f_{0-}\right]\left[d_{2}^{+} d_{1}-d_{1}^{+} d_{2}\right]- \\
& -\frac{\Delta}{2}\left[d_{1}^{+} d_{2}+d_{2}^{+} d_{1}\right]
\end{aligned}
$$

O Hamiltoniano está discretizado, porém, temos a BC expressa em função de uma série infinita. Por isso, não pode ser implementado computacionalmente. $O$ próximo passo no GRN é determinar um critério para que possamos truncar a série.

\section{3 - Truncamento}

A BC está expressa por uma série infinita dos operadores $a_{p}, b_{p}$, etc. e todos eles se acoplam com o ín. Para que possamos truncar a série, vamos seguir o procedimento de Wilson para uma impureza [14] e fazer uma transformação unitária do conjunto dos operadores $\left(a_{p}, b_{p}, a_{p m}, b_{p m}\right)$ para um novo conjunto de operadores ortonormais $\left(f_{0 p}, f_{1 p}, \ldots, f_{n p}, \ldots\right)$, onde $f_{0_{p}}$ continua a ser dado pela Eq. (3.43), e é o único a se acoplar com o íon. Os demais operadores se acoplam somente com seus vizinhos mais próximos, ou seja, $f_{n p}$ se acopla com $f_{n \pm 1, p}[14]$. Com esses operadores expressamos a BC da seguinte forma 


$$
H_{b c}=\sum_{n=0}^{\infty} \varepsilon_{n}^{z}\left(f_{n,+}^{+} f_{n+1,+}+f_{n,-}^{+} f_{n+1,-}+\text { h.c. }\right)
$$

onde h.c. é o hermitiano conjugado. Dessa forma o Hamiltoniano $H$ da Eq.(3.44) fica

$$
\begin{aligned}
H= & \sum_{n=0}^{\infty} \varepsilon_{n}^{z}\left(f_{n,+}^{+} f_{n+1,+}+f_{n,-}^{+} f_{n+1,-}+\text { h.c. }\right)+ \\
& +2 G\left[A_{+}^{2} f_{0+}^{+} f_{0+}+A_{-}^{2} f_{0-}^{+} f_{0-}\right]\left[d_{1}^{+} d_{1}+d_{2}^{+} d_{2}\right]+ \\
& +2 G A_{+} A_{-}\left[f_{0+}^{+} f_{0-}+f_{0-}^{+} f_{0+}\right]\left[d_{1}^{+} d_{1}-d_{2}^{+} d_{2}\right]+ \\
& +2 G_{a}\left[A_{+}^{2} f_{0+}^{+} f_{0+}-A_{-}^{2} f_{0-}^{+} f_{0-}\right]\left[d_{2}^{+} d_{1}+d_{1}^{+} d_{2}\right]+ \\
& +2 G_{a} A_{+} A_{-}\left[f_{0-}^{+} f_{0+}-f_{0+}^{+} f_{0-}\right]\left[d_{2}^{+} d_{1}-d_{1}^{+} d_{2}\right]- \\
& -\frac{\Delta}{2}\left[d_{1}^{+} d_{2}+d_{2}^{+} d_{1}\right] .
\end{aligned}
$$

Para $z=1$, os coeficientes $\varepsilon_{n}^{z}$ podem ser obtidos através da seguinte expressão [14]

$$
\varepsilon_{n}=\Lambda^{-n / 2}\left[1-\Lambda^{-(n+1)}\right]\left[1-\Lambda^{-2 n+1}\right]^{-1 / 2}\left[1-\Lambda^{-(2 n+3)}\right]^{-1 / 2}\left[1+\Lambda^{-1}\right] / 2 .
$$

Para os demais valores de $z$, os coeficientes $\varepsilon_{n}^{z}$ são obtidos numericamente [27], sendo que para $n$ grande ele apresenta o seguinte comportamento assintótico

$$
\varepsilon_{n}^{z} \cong \frac{1+\Lambda^{-1}}{2} \Lambda^{1-z-\frac{n}{2}}, \quad \mathrm{n}>>1
$$

ou seja, quanto maior $n$ menor o elemento de matriz que conecta $f_{n} \operatorname{com} f_{n+1}$. Isso permite que trunquemos a série em $n=N$. Os valores de $N$ utilizados nessa dissertação são da ordem de 20 para $\Lambda$ em torno de 4 , ou seja, esses são valores que permitem obter toda a curva de calor específico no intervalo de temperatura desejado.

A série truncada é dada por: 


$$
H_{b c}^{N}=\sum_{n=0}^{N-1} \varepsilon_{n}^{z}\left(f_{n,+}^{+} f_{n+1,+}+f_{n,-}^{+} f_{n+1,-}+\text { h.c. }\right)
$$

É conveniente, por motivos computacionais, fazermos com que o menor termo da série truncada seja da ordem da unidade. Por isso, vamos multiplicar o Hamiltoniano completo, Eq. (3.46), pelo fator

$$
\frac{2}{1+\Lambda^{-1}} \Lambda^{\frac{N-1}{2}}
$$

Além disso, vamos expressar os operadores $d_{1}, d_{2}$ em função de operadores com paridade definida, tal que

$d_{+}=\frac{d_{1}+d_{2}}{\sqrt{2}} \rightarrow$ combinação ligante (par)

$d_{-}=\frac{d_{1}-d_{2}}{\sqrt{2}} \rightarrow$ combinação antiligante (ímpar).

Assim, o Hamiltoniano do sistema fica

$$
\begin{aligned}
H_{N} & =\Lambda^{\frac{N-1}{2}\left\{\sum_{n=0}^{N-1} \bar{\varepsilon}_{n}^{z}\left[f_{n,+}^{+} f_{n+1,+}+f_{n,-}^{+} f_{n+1,-}+\text { h.c. }\right]+\right.} \\
& +2 \bar{G}\left[A_{+}^{2} f_{0+}^{+} f_{0+}+A_{-}^{2} f_{0-}^{+} f_{0-}\right]\left[d_{+}^{+} d_{+}+d_{-}^{+} d_{-}\right]+ \\
& +2 \bar{G} A_{+} A_{-}\left[f_{0+}^{+} f_{0-}+f_{0-}^{+} f_{0+}\right]\left[d_{+}^{+} d_{-}+d_{-}^{+} d_{+}\right]+ \\
& +2 \bar{G}_{a}\left[A_{+}^{2} f_{0+}^{+} f_{0+}-A_{-}^{2} f_{0-}^{+} f_{0-}\right]\left[d_{+}^{+} d_{+}-d_{-}^{+} d_{-}\right]+ \\
& +2 \bar{G}_{a} A_{+} A_{-}\left[f_{0-}^{+} f_{0+}-f_{0+}^{+} f_{0-}\right]\left[d_{+}^{+} d_{-}-d_{-}^{+} d_{+}\right]- \\
& \left.-\frac{\bar{\Delta}}{2}\left[d_{+}^{+} d_{+}-d_{-}^{+} d_{-}\right]\right\},
\end{aligned}
$$

onde

$$
\bar{\varepsilon}_{n}^{z}=\frac{2 \varepsilon_{n}^{z}}{1+\Lambda^{-1}}, \bar{G}=\frac{2 G}{1+\Lambda^{-1}}, \bar{G}_{a}=\frac{2 \bar{G}_{a}}{1+\Lambda^{-1}} \text { e } \bar{\Delta}=\frac{2 \Delta}{1+\Lambda^{-1}} .
$$


$H_{N}$ é o Hamiltoniano completo discretizado, truncado e escalado. Na seção 3.5 vamos explicar o processo de resolução iterativo desse Hamiltoniano.

Podemos observar em $H_{N}$ que a BC é escrita em termos de operadores com paridade definida, pois os elétrons que interagem com o ín são apenas aqueles cujas funções de onda têm paridade definida. A BC pode ser interpretada, então, como tendo dois canais: o canal par, que possui elétrons com função de onda par; e o canal impar, que possui elétrons com função de onda ímpar.

O Hamiltoniano $H$, Eq.(3.46), pode ser recuperado fazendo-se

$$
H=\lim _{N \rightarrow \infty} \frac{1+\Lambda^{-1}}{2} \Lambda^{-\frac{N-1}{2}} H_{N}
$$

Devido ao fator de escala (3.50) devemos escalar qualquer grandeza que seja comparada com as excitações da banda. Em nosso caso, temos a temperatura como grandeza de interesse, sendo que ela aparece no fator de Boltzmann $\exp (-\beta H)$ no cálculo do calor específico. Como temos o espectro de $H_{N}$, e não de $H$, devemos calcular expressões do tipo

$$
\exp \left(-\beta D \frac{1+\Lambda^{-1}}{2} \Lambda^{-\frac{N-1}{2}} H_{N}\right)
$$

Para isso, nós definimos uma variável adimensional $\bar{\beta}$ e deixamos para escalar a temperatura como,

$$
\exp \left(-\beta D \frac{1+\Lambda^{-1}}{2} \Lambda^{-\frac{N-1}{2}} H_{N}\right) \equiv \exp \left(-\bar{\beta} H_{N}\right)
$$

onde $\bar{\beta}$ é um número que deve ser escolhido próximo à unidade, já que os menores autovalores de $H_{N}$ são da ordem da unidade. Dessa forma, temos 


$$
\frac{1}{k_{B} T_{N}} D \frac{1+\Lambda^{-1}}{2} \Lambda^{-\frac{N-1}{2}}=\bar{\beta} \rightarrow T_{N}=\frac{1}{k_{B} \bar{\beta}} D \frac{1+\Lambda^{-1}}{2} \Lambda^{-\frac{N-1}{2}} .
$$

Com essa relação obtemos a temperatura correspondente à faixa de energia do intervalo considerado. A relação (3.58) mostra que quanto maior o valor de $N$ menor a temperatura associada a ele.

\section{4 - As Simetrias do Problema}

O processo de diagonalização é facilitado pelas simetrias do Hamiltoniano, que definem as grandezas que são conservadas.

No modelo que estamos tratando, não é permitida a troca de carga entre a BC e o íon tunelante. Esse fato se reflete no Hamiltoniano através da conservação da carga do ion e pela conservação da carga da banda de condução.

Isto pode ser verificado observando que o operador número de partículas do ín

$$
Q_{I}=d_{1}^{+} d_{1}+d_{2}^{+} d_{2}
$$

comuta com $H_{N}$. Em nosso modelo, a carga líquida do íon se mantém constante e igual a um.

Para a BC temos que o operador número de partículas

$$
Q_{N}=\sum_{n=0}^{N}\left(f_{n,+}^{+} f_{n,+}+f_{n,-}^{+} f_{n,-}\right)
$$

também comuta com $H_{N}$, conservando o número total de partículas.

Uma terceira característica do modelo é a conservação da paridade, que vem do fato do Hamiltonano ficar invariante sobre a troca de posição dos mínimos de potencial $(\vec{R} / 2 \rightarrow-\vec{R} / 2)$ e a troca de $d_{1}$ por $d_{2}$. Daí vem o motivo de termos escrito $H_{N}$ (Eq. 3.53) em função apenas de operadores com paridade definida. 
Trocando em miúdos, a conservação da paridade nos diz que se o íon tunelante troca de paridade a $\mathrm{BC}$ como um todo também troca.

\section{5 - Diagonalização Iterativa}

A estrutura da Eq. (3.53) permite que se obtenha a seguinte relação de recorrência:

$H_{N+1}=\Lambda^{1 / 2} H_{N}+\xi_{N}\left(f_{N,+}^{+} f_{N+1,+}+f_{N,-}^{+} f_{N+1,-}+f_{N+1,+}^{+} f_{N,+}+f_{N+1,-}^{+} f_{N,-}\right)$

onde

$$
\xi_{N}=\Lambda^{N / 2} \varepsilon_{N}^{z}
$$

A Eq. (3.61) é o ponto central do formalismo discutido até agora e é conhecida como Transformação do Grupo de Renormalização, sendo que ela nos permite diagonalizar Hamiltonianos não quadráticos de uma forma iterativa.

Para iniciar o processo iterativo, diagonalizamos analiticamente $H_{0}$, dado por

$$
\begin{aligned}
\Lambda^{1 / 2} H_{0}=2 \bar{G}\left[A_{+}^{2} f_{0+}^{+} f_{0+}+A_{-}^{2} f_{0-}^{+} f_{0-}\right]\left[d_{+}^{+} d_{+}+d_{-}^{+} d_{-}\right]+ \\
+2 \bar{G} A_{+} A_{-}\left[f_{0+}^{+} f_{0-}+f_{0-}^{+} f_{0+}\right]\left[d_{+}^{+} d_{-}+d_{-}^{+} d_{+}\right]+ \\
+2 \bar{G}_{a}\left[A_{+}^{2} f_{0+}^{+} f_{0+}-A_{-}^{2} f_{0-}^{+} f_{0-}\right]\left[d_{+}^{+} d_{+}-d_{-}^{+} d_{-}\right]+ \\
+2 \bar{G}_{a} A_{+} A_{-}\left[f_{0-}^{+} f_{0+}-f_{0+}^{+} f_{0-}\right]\left[d_{+}^{+} d_{-}-d_{-}^{+} d_{+}\right]- \\
\quad-\frac{\bar{\Delta}}{2}\left[d_{+}^{+} d_{+}-d_{-}^{+} d_{-}\right]
\end{aligned}
$$

A base mais conveniente para diagonalizarmos $H_{0}$ é dada por $\left|f_{0+} f_{0-} ; d_{+} d_{-}\right\rangle$. Nessa base os operadores do Hamiltoniano $H_{0}$ atuam da seguinte forma: $f_{0+(-)}$ destrói um elétron da $\mathrm{BC}$ com paridade par (ímpar), $d_{+(-)}$ destrói o íon no estado ligante (antiligante). Em nosso modelo, o íon está com uma 
carga líquida igual a um. Por isso, restringiremos nossa base para autofunções em que a carga líquida do íon é igual a 1 . Os vetores dessa base são

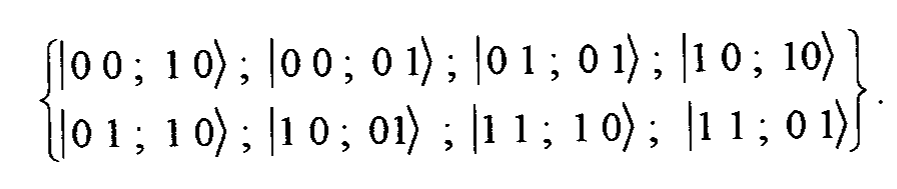

Dos vetores apresentados em (3.64), o primeiro, terceiro, quarto e oitavo são pares, os demais ímpares. Assim, utilizamos a conservação de paridade e carga da BC para simplificar nosso trabalho e construímos uma matriz $8 \times 8$ bloco diagonalizada, onde o maior bloco é apenas $2 \times 2$ que é facilmente diagonalizado.

Feita a diagonalização de $H_{0}$, devemos a partir do seus auto-estados, construir a base para diagonalizar $H_{1}$ e assim sucessivamente. Para isso, é mais conveniente deixarmos a representação $\left|f_{0+} f_{0-} ; d_{+} d_{-}\right\rangle$e trabalharmos com uma nova representação dada por

$$
|N Q, N P, m\rangle_{N}
$$

onde $N Q$ é o número de cargas da $\mathrm{BC}, N P$ é paridade do estado ( $N P=0 \rightarrow$ par, $N P=1 \rightarrow$ ímpar), $m$ é o índice para diferenciar os estados com $N Q$ e $N P$ iguais e $N$ é o sub-índice que indica se a base é de $H_{0}, H_{1}$, etc.

Agora, para construirmos a base de $H_{N+1}$, partimos da base de $H_{N}$, sendo que cada um dos auto-estados deste gera quatro vetores de $H_{N+1}$. Isso porque da passagem de $H_{N}$ para $H_{N+1}$ podemos não acrescentar nenhum elétron de condução a um dado autovetor de $H_{N}$, acrescentar um elétron par ou um elétron ímpar e a última opção possível é colocar dois elétrons, sendo um par e um ímpar, de acordo com o seguinte esquema 


$$
\begin{aligned}
& \mathrm{I}|\mathrm{NQ} \mathrm{NP} \mathrm{m}\rangle_{N}=|\mathrm{NQ}-1 \mathrm{NP} \overline{\mathrm{m}}\rangle_{N+1} \rightarrow \text { Tipo } 1 \\
& f_{N+1,+}^{+}|\mathrm{NQ} \mathrm{NP} \mathrm{m}\rangle_{N}=|\mathrm{NQ} \mathrm{NP} \overline{\mathrm{m}}\rangle_{N+1} \rightarrow \text { Tipo 2 } \\
& f_{N+1,-}^{+}|\mathrm{NQ} \mathrm{NP} \mathrm{m}\rangle_{N}=|\mathrm{NQ} \mathrm{NP} \overline{\mathrm{m}}\rangle_{N+1} \rightarrow \text { Tipo } 3 \\
& f_{N+1,+}^{+} f_{N+1,-}^{+}|\mathrm{NQ} \mathrm{NP}\rangle_{N}=|\mathrm{NQ}+1 \mathrm{NP} \overline{\mathrm{m}}\rangle_{N+1} \rightarrow \text { Tipo 4, }
\end{aligned}
$$

onde I significa identidade. A redefinição do número de carga na base da iteração $N+1$ é apenas por conveniência computacional.

Cada um dos processos acima é identificado pelo tipo, 1, 2, 3 ou 4, conforme indicado. NP significa paridade oposta a NP. A cada etapa do processo iterativo temos quatro vezes mais estados que a etapa anterior. Esta progressão gera um problema computacional, pois precisamos lidar com matrizes cada vez maiores na diagonalização. Por exemplo, para $\mathrm{N}=4$ teremos uma matriz com dimensão $2048 \times 2048$. Mesmo podendo dividir esta matriz em sub-espaços menores, utilizando as simetrias do Hamiltoniano, teremos problema com tamanho das matrizes. Por isso, devemos realizar um corte nas nossas auto-energias, isto é, energias maiores que certo valor são desconsideradas e os auto-estados correspondentes não são usados para se encontrar a base da próxima iteração. Esse procedimento não afeta nossos resultados, desde que estamos interessados em propriedades em baixas temperaturas.

\section{6 - A Transformação do GRN e os Pontos Fixos}

Nesta seção falaremos um pouco mais sobre a transformação do Grupo de Renormalização Numérico, e também faremos uma breve descrição dos pontos fixos dessa transformação.

Como vimos na seção 3.5 o GRN é baseado na transformação dada pela Eq.(3.61)

$$
H_{N+1}=\Lambda^{1 / 2} H_{N}+\xi_{N}\left(f_{N,+}^{+} f_{N+1,+}+f_{N,-}^{+} f_{N+1,-}+f_{N+1,+}^{+} f_{N,+}+f_{N+1,-}^{+} f_{N,-}\right),
$$


que permite definir o processo iterativo da diagonalização. A relação (3.59) pode ser representada genericamente por $\mathcal{J}\left[H_{N}\right]=H_{N+1}$. Um ponto fixo $H^{*}$ da transformação $\mathcal{J}$ é um Hamiltoniano que se mantêm invariante sobre essa transformação, ou seja, $\mathcal{T}\left[H^{*}\right]=H^{*}$. Na verdade, no GRN os pontos fixos são dados pela aplicação do operador $\boldsymbol{J}^{2}$ (aplicação do operador $\boldsymbol{T}$ duas vezes), isto é, $\nearrow^{2}\left[H_{N}\right]=H_{N+2}$.

Um ponto fixo de $\boldsymbol{T}^{2}$ é caracterizado numericamente quando um conjunto das auto-energias de muitos corpos se repete (ou, na prática, muda muito pouco) quando realizamos o processo iterativo duas vezes. Nesse processo quando $N$ aumenta o Hamiltoniano muda de um ponto fixo inicial para um ponto fixo final. $O$ estudo detalhado dos pontos fixos é um tópico bastante interessante e muitas informações a respeito do sistema podem ser obtidas através dele. Porém, um aprofundamento neste estudo está fora do alcance dessa dissertação e apenas mostraremos alguns exemplos de ponto fixo. Para o leitor mais interessado nesse assunto aconselhamos as refs. [15,24,28]

Nas próximas análises, por simplicidade, nos restringiremos ao caso $z=1$, sendo, neste caso, $\varepsilon_{n}^{z}$ denotado abreviadamente por $\varepsilon_{n}$. As conclusões são, no entanto, independentes de $z$.

\subsection{1 - Banda Livre}

O primeiro ponto fixo que analisaremos é o da BC livre, cujo Hamiltoniano é dado por

$$
H_{b c}^{N}=\sum_{n=0}^{N-1} \varepsilon_{n}\left(f_{n,+}^{+} f_{n+1,+}+f_{n,-}^{+} f_{n+1,-}+\text { h.c. }\right)
$$

$H_{B C}^{N}$ é um Hamiltoniano quadrático e portanto de partícula independente. Para diagonalizá-lo, podemos usar procedimentos mais simples que o iterativo 
definido pelo GRN. Para isso, vamos considerar apenas o canal par da BC, sendo que o canal ímpar tem tratamento análogo.

Neste caso, definimos o vetor linha $\hat{f}^{+}=\left(f_{0}^{+}, f_{1}^{+}, \ldots, f_{N}^{+}\right)$, e o vetor coluna conjugado $\hat{f}$. Temos, então, que

$$
H_{b c}^{N}=\hat{f}^{+} \#_{b c}^{N} \hat{f}
$$

onde a matriz $\mathbb{Z}_{b c}^{N}$, de dimensão $(N+1) \times(N+1)$, é tridiagonal, sendo seus elementos dados por

$$
\left[\mathbb{F}_{b c}^{N}\right]_{n, m}=\varepsilon_{n-1} \delta_{n+1, m}+\varepsilon_{n-2} \delta_{n, m+1}, \quad n, m=1,2, \ldots, N+1
$$

A matriz $Z_{b c}^{N}$ é agora facilmente diagonalizada.

A tabela 3.1 mostra os resultados para $N$ ímpar, onde omitimos os autovalores negativos, pois temos $(N+1) / 2$ pares de autovalores simétricos em relação ao nível de Fermi como resultado.

\begin{tabular}{|c|c|c|c|c|c|c||}
\hline \hline & $n_{1}^{-}$ & $n_{2}^{-}$ & $n_{3}^{-}$ & $n_{4}^{-}$ & $n_{5}^{-}$ & $n_{6}^{-}$ \\
\hline 1 & 0.87287 & & & & & \\
\hline 3 & 0.85978 & 3.99952 & & & & \\
\hline 5 & 0.85895 & 3.99945 & 15.99999 & & & \\
\hline 7 & 0.85895 & 3.99945 & 15.99999 & 64.00000 & & \\
\hline 9 & 0.85891 & 3.99964 & 16.00001 & 64.00000 & 256.0000 & \\
\hline 11 & 0.85891 & 3.99950 & 16.00075 & 64.00075 & 256.0000 & 1024.000 \\
\hline
\end{tabular}

Tabela 3.1: Autovalores de $H_{b c}^{N}$ para N ímpar e $\Lambda=4$.

O caso de $N$ par, mostrado na tabela 3.2, é um pouco diferente, pois um dos autovalores é nulo e as demais $\mathrm{N} / 2$ energias são simétricas em torno da energia zero. 


\begin{tabular}{|c|c|c|c|c|c|c||}
\hline$N$ & $n_{0}^{+}$ & $n_{1}^{+}$ & $n_{2}^{+}$ & $n_{3}^{+}$ & $n_{4}^{+}$ & $n_{5}^{+}$ \\
\hline 2 & 0.0 & 1.98528 & & & & \\
\hline 4 & 0.0 & 1.98341 & 7.99999 & & & \\
\hline 6 & 0.0 & 1.98328 & 7.99999 & 31.99999 & & \\
\hline 8 & 0.0 & 1.98337 & 8.00000 & 32.00000 & 128.000 & \\
\hline 10 & 0.0 & 1.98328 & 8.00009 & 32.00150 & 128.000 & 512.000 \\
\hline
\end{tabular}

Tabela 3.2: Autovalores de $H_{b c}^{N}$ para N par e $\Lambda=4$.

Podemos observar nas tabelas 3.1 e 3.2 que, ao se aumentar o valor de $N$, os autovalores para $N$ impar e para $N$ par, denotados por $n_{l}^{-}$e $n_{l}^{+}$, respectivamente, tendem aos limites

$n_{l}^{-}= \pm \Lambda^{l-z}, \quad$ onde $l=1,2, \ldots,(N+1) / 2$

e

$n_{l}^{+}= \pm \Lambda^{l-z+\frac{1}{2}}, \quad l=1,2, \ldots, N / 2$ e $n_{0}^{+}=0$.

sendo que $z$ foi acrescentado para englobar o caso geral.

Esses limites são explicados pela forma como a BC foi discretizada, escalada e truncada. Na escala discreta, a razão entre duas energias consecutivas é $\Lambda$, então os autovalores se distribuem entre o maior e o menor valor de forma que a razão entre dois autovalores sucessivos seja $\Lambda$. O menor autovalor do Hamiltoniano escalado e truncado é $-\Lambda^{(N-1) / 2}$ e o maior $\Lambda^{(N-1) / 2}$ (para $z=1$ e $N$ grande). Dividimos esses valores por $\Lambda$ sucessivamente até chegarmos às menores escalas de energia da $\mathrm{BC}$ discretizada que na escala escolhida são: $\approx 1$ se $N$ for impar e $\approx \sqrt{\Lambda}$ se $N$ for par ( $N$ par inclui a energia zero). 
Os autovalores da $H_{B C}^{N}$ ilustram o processo de ponto fixo, pois podemos perceber claramente que as energias se mantêm quando aumentamos de 2 em 20 valor de $N$, com exceção do topo da banda. Este é o ponto fixo de banda livre.

Pode-se mostrar que a presença de um potencial eletrostático $G$, devido a um centro espalhador, apenas desloca os níveis de energia de acordo com as expressões [29]

$n_{l}^{-}= \pm \Lambda^{l-z \mp \frac{\delta}{\pi}} \quad$ onde $l=1,2, \ldots,(N+1) / 2$

$$
n_{l}^{+}= \pm \Lambda^{l-z+\frac{1}{2} \mp \frac{\delta}{\pi}}, \quad l=1,2, \ldots, N / 2 \text { e } n_{0}^{+}=0
$$

onde $\delta$ é a defasagem das funções de onda da banda na presença do potencial $G$ e é dado por

$$
\operatorname{tg}(\delta)=\frac{-\pi G}{D}
$$

Assim, não temos mais os autovalores distribuídos simetricamente em torno do nível de Fermi, ou seja, não temos simetria partícula-buraco.

Por outro lado, a presença de um sistema de dois níveis introduz uma escala de energia característica $\Delta$, que tende a levar o sistema para outro ponto fixo

A Figura 3.4 ilustra esse processo. Neste gráfico mostramos as energias mais baixas do Hamiltoniano (3.53) para o caso de $\mathrm{N}$ ímpar. Nele temos um primeiro patamar de pontos fixos que vai até aproximadamente $N=7$. Neste ponto se inicia uma região de cruzamento que vai até aproximadamente $N=17$, onde se inicia o segundo patamar.

Esse gráfico é entendido da seguinte maneira: para pequenos valores de $N$, ou equivalentemente grandes temperaturas, o sistema não percebe a presença da energia do sistema de dois níveis $\Delta$, pois a energia térmica $k_{B} T$ associada àquela temperatura é muito grande comparada à $\Delta$. À medida que $N$ aumenta, a energia térmica diminui e fica da ordem de $\Delta$, neste momento o sistema de dois níveis passa a 


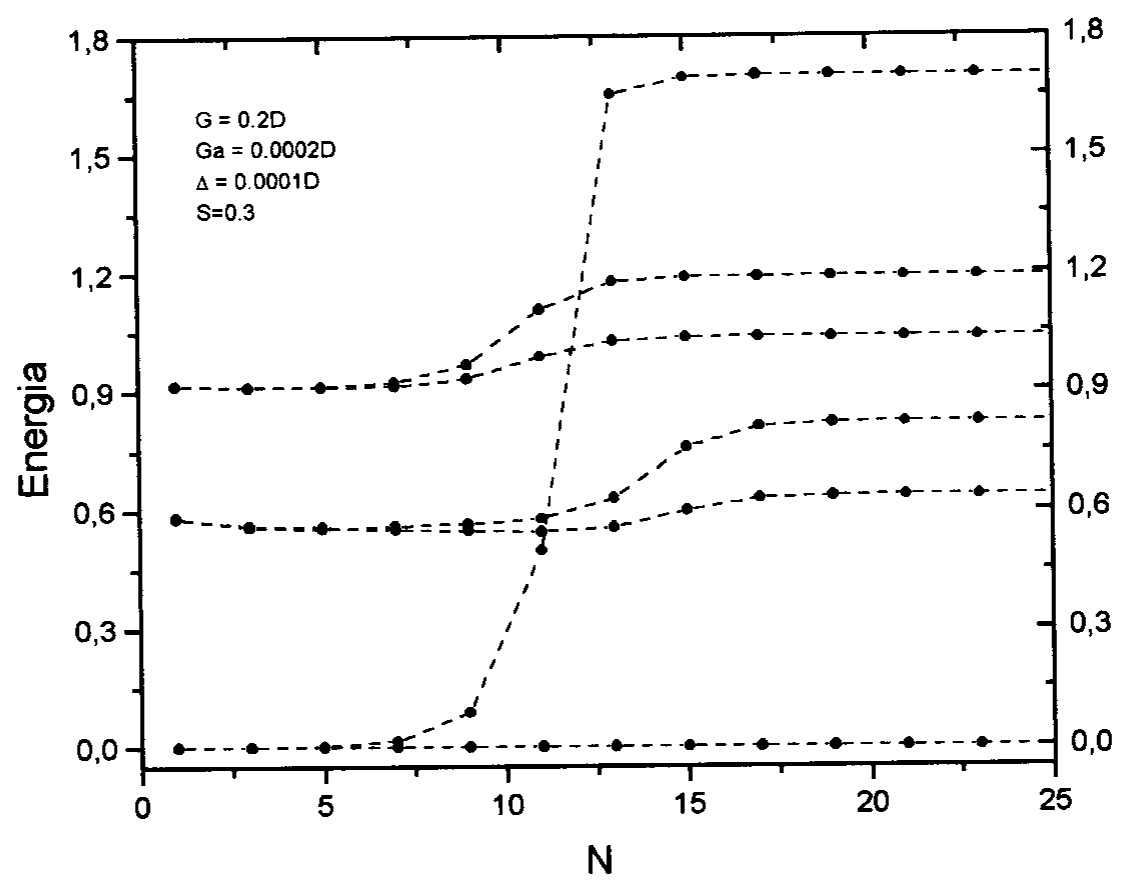

Figura 3.4: Diagrama de fluxo de energia do modelo de Kondo de Tunelamemento, mostrando a evolução das energias mais baixas para $\mathrm{N}$ ímpar. A presença da escala de energia característica $\Delta$ quebra a invariância de escala da $\mathrm{BC}$, levando as energias de um ponto fixo para outro.

ser importante e o sistema total entra num processo de "crossover". Para $N$ muito grande $(N>17), k_{B} T$ é muito menor que $\Delta$ e o sistema entra em um novo ponto fixo (chamado de ponto fixo de $\Delta$ infinito). Como não temos nenhuma escala de energia do sistema de dois níveis menor que $\Delta$ o sistema ficará estável nesse novo ponto fixo. 


\section{Capítulo 4}

\section{Resultados}

\section{1 - Calor Específico}

A contribuição do íon tunelante para o calor específico, $C_{i o n}$, é definido como o calor específico do sistema íon + metal menos o calor específico dos elétrons de condução do metal puro. Podemos escrever sua expressão em termos da energia livre do íon, $F_{\text {ion }}$, como:

$$
C_{i o n}=-T \frac{\partial^{2}}{\partial T^{2}} F_{i o n}
$$

sendo

$$
F_{\text {ion }}=-k_{B} T \lim _{N \rightarrow \infty}\left(\ln \operatorname{Tr} e^{-\bar{\beta} H_{N}}-\ln \operatorname{Tr} e^{-\bar{\beta} H_{b c}}\right)
$$

onde $H_{N}$ é dado pela Eq.(3.53), e $H_{b c}$ pela Eq.(3.45). O termo $\bar{\beta}$ foi definido na Eq. (3.58) e é dado por

$$
\bar{\beta}=\frac{D}{k_{B} T_{N}} \frac{1+\Lambda^{-1}}{2} \Lambda^{-\frac{N-1}{2}}
$$

Este calor específico é calculado no ensemble grand-canônico onde fazemos o potencial químico igual a zero. 


$$
\frac{C_{i o n}}{k_{B}}=\bar{\beta}^{2}\left[\left\langle E^{2}\right\rangle-\langle E\rangle^{2}-\left\langle E^{2}\right\rangle_{0}+\langle E\rangle_{0}^{2}\right] \equiv C-C_{0},
$$

onde \langle\rangle significa a média termodinâmica sobre os estados do íon e de condução e \langle\rangle$_{0}$ a média sobre os estados de condução. Os valores médios das energias são definidos como

$$
\langle E\rangle=\frac{\operatorname{Tr} H_{N} e^{-\bar{\beta} H_{N}}}{\operatorname{Tr} e^{-\bar{\beta} H_{N}}},
$$

e

$$
\langle E\rangle_{0}=\frac{\operatorname{Tr} H_{b c} e^{-\bar{\beta} H_{b c}}}{\operatorname{Tr} e^{-\bar{\beta} H_{b c}}}
$$

Da diagonalização numérica de $H_{N}$ obtemos seu espectro de energia, o que permite o cálculo das médias presentes na Eq. (4.3). $\mathrm{O}$ cálculo de $C_{0}$ é trivial, já que o termo do Hamiltoniano que representa a $\mathrm{BC}$ do metal é quadrático.

Como parâmetros de entrada de nosso programa temos $G, G_{a}, \Delta$ e $S$. O Parâmetro $G$ é a transformada de Fourier da interação Coulombiana e foi definida no Capitulo 2, Eq. (2.15), como:

$$
V(\vec{k},-\vec{k})=G \rightarrow \text { constante }
$$

onde usamos o fato da blindagem eletrostática dos elétrons de condução limitar o seu alcance, permitindo que fosse considerado constante. Se $G$ for menor que zero, significa que o íon está com uma carga eletrônica a menos e o potencial é de atração. Quando o íon está com um elétron a mais o potencial é de repulsão e $G$ é maior que zero. Zawadowski et. al. estimam $G$ como sendo da ordem de $0.2 D$ para que a temperatura que separa a região de fraco e forte acoplamento do íon com a BC esteja numa região de interesse físico $(T>1 K)$ [7]. 
O parâmetro $G_{a}$ representa o tunelamento assistido pelos elétrons de condução e, a princípio, pode tomar valores positivos ou negativos. Seguindo as estimativas feitas por Zawadowski et. al. a razão $G_{a} / G$ é da ordem de $10^{-4}$ a $10^{-3}$ quando $\Delta$ é da ordem de $10^{-4} D[7] . \Delta$ é o parâmetro que representa a taxa de tunelamento livre do íon (sem interação com a BC do metal). Seu valor é considerado positivo para que tenhamos o estado ligante como estado fundamental do ín. Quando $G=0$ e $G_{a}=0$ não temos espalhamento nem tunelamento assistido e a taxa efetiva de tunelamento $\left(\Delta^{*}\right)$ é igual a taxa de tunelamento livre. Os parâmetros $G, G_{a}$ e $\Delta$ são expressos em termos da meia largura da $\mathrm{BC}, D$, sendo que os metais possuem $D$ na faixa de aproximadamente $5 \mathrm{eV}$ a $10 \mathrm{eV}$.

Finalmente, o parâmetro $S$ é definido como:

$$
S=\frac{\operatorname{sen}\left(k_{F} R\right)}{k_{F} R}
$$

onde $k_{F} \equiv k\left(\varepsilon_{F}\right)$ é o módulo do momento do elétron de condução no nível de Fermi e $R$ é a distância entre os mínimos de potencial . O parâmetro $S$ tem origem na Eq.(3.10) e dá uma noção da distância entre os mínimos. Quando $S=0$ temos os mínimos infinitamente afastados um do outro $(R \rightarrow \infty)$, ou com $k_{F} R$ em um dos zeros da função seno. Quando $S=1$ temos os mínimos localizados no mesmo sítio.

Nas próximas seções mostraremos os resultados para $C_{i o n}$ em função de $k_{B} T / D$ quando variamos os parâmetros mencionados acima. Começaremos na próxima seção com o caso em que $G_{a}=0$.

\section{2 - Calor Específico sem Tunelamento Assistido}

Quando não há interação entre as impurezas e a banda de condução, isto é, quando temos os parâmetros $G=0$ e $G_{a}=0, C_{i o n}$ é o de um sistema de dois níveis simples e é dado por 


$$
\frac{C_{\text {ion }}}{k_{B}}=(\beta \Delta)^{2} \frac{e^{-\beta \Delta}}{\left(1+e^{-\beta \Delta}\right)^{2}},
$$

sendo que o elemento de matriz que acopla os dois níveis é $\Delta / 2$. Na Figura 4.1 mostramos a curva que representa a expressão (4.8) através de uma linha cheia. Os pontos sobre a linha são os resultados obtidos pelo GRN na condição em que não há interação entre o íon e a BC.

A interpretação para esse resultado é a seguinte: para baixas temperaturas, muito menores que $\Delta / k_{B}$, o estado antiligante não é acessível e, portanto, $C_{i o n}$ vai a zero. Com o aumento da temperatura, o estado antiligante passa a ser populado, refletindo no calor específico como um pico. Para temperaturas maiores que $\Delta$, como não temos nenhum outro estado acessível para o íon, o calor específico decresce.

Utilizando a expressão (4.8) podemos determinar a temperatura $\left(T_{P}\right)$ na qual ocorre o pico do calor específico (posição de máximo da curva):

$$
\frac{k_{B} T_{P}}{D} \cong 0.417 \Delta
$$

$\mathrm{Na}$ mesma Fig. 4.1 mostramos uma curva para o caso em que $G \neq 0$ e $G_{a}=0$, ou seja, quando temos interação eletrostática entre íon tunelante e elétrons da $\mathrm{BC}$ (o valor de $G$ pode ser positivo ou negativo que teremos a mesma curva). Percebemos que, neste caso, o pico se desloca para temperaturas mais baixas, indicando que houve uma renormalização na taxa de tunelamento. Este efeito é característico do parâmetro $G$ e quanto maior seu valor, mais o pico se desloca para baixas temperaturas. Podemos entender esse resultado percebendo que com $G \neq 0$ os estados ligante e antiligante do íon não são mais auto-estados do Hamiltoniano, tendo, portanto, um alargamento das linhas de energia, fazendo com que a diferença mínima de energia entre esses estados diminua de $\Delta$ para $\Delta^{*}$ (Fig.4.2). Outra maneira de interpretar esse resultado é que agora o íon ao tunelar tem que arrastar a $\mathrm{BC}$ e, portanto, é como se ele tivesse uma massa efetiva maior o que dificulta $o$ tunelamento. 


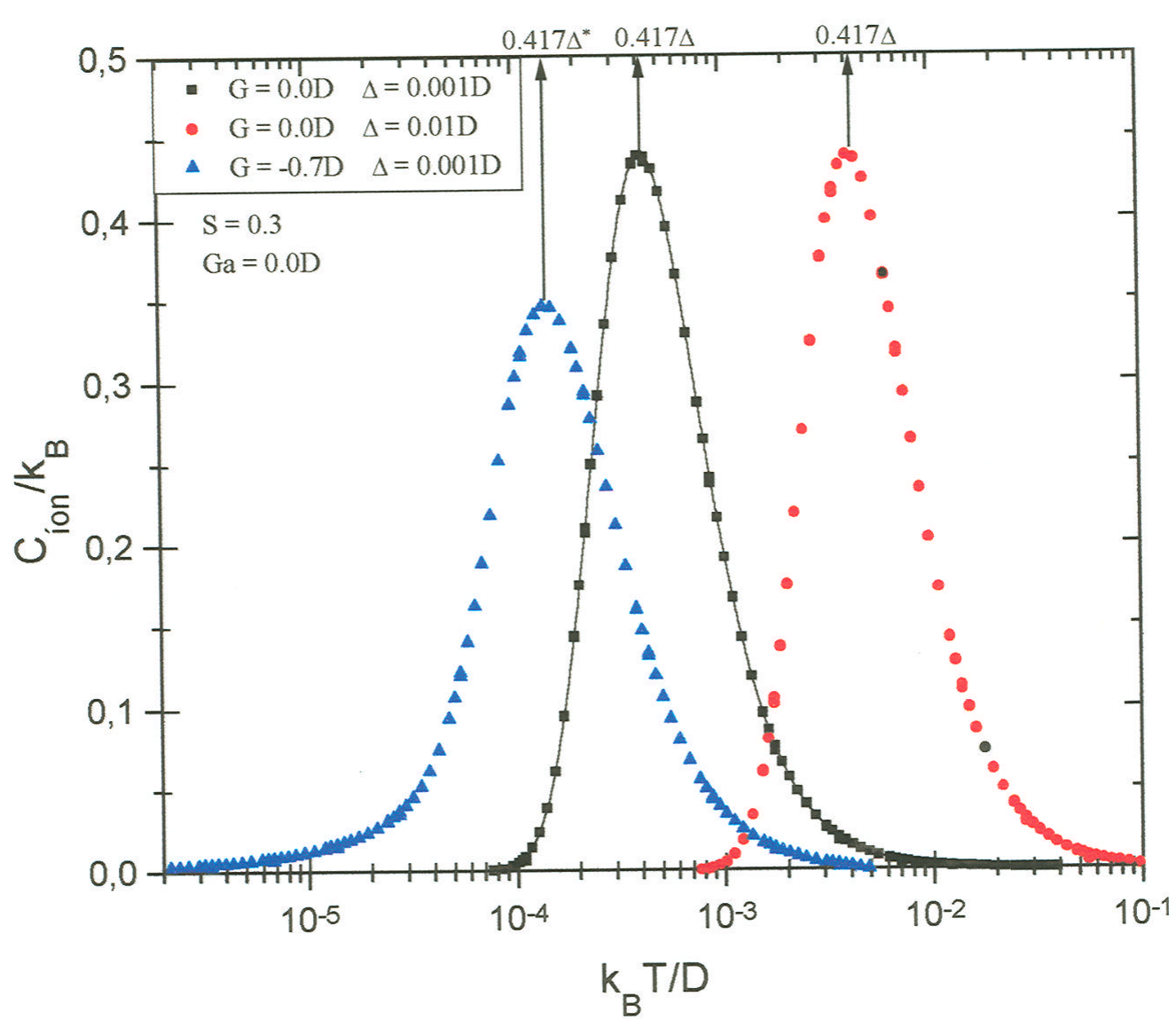

Figura 4.1: Curvas de calor específico com valores diferentes de $G$ e $\Delta$ e com $G_{a}=0$. Quando $G=0$ a taxa de tunelamento não é alterada e o pico encontra-se no valor de energia $0.417 \Delta$, como mostra a curva com pontos pretos (a linha cheia é a curva teórica dada pela Eq. 4.6). Quando $G \neq 0$ ( $G$ é menor que zero pois a interação é entre um íon que está com uma carga elêtronica a menos e os elétrons de condução), o pico ocorre em outro valor de energia. Dizemos que a taxa de tunelamento é renormalizada para um valor $\Delta^{*}$ tal que a nova posição do pico ocorra em $0.417 \Delta^{*}$ (curva azul). A curva vermelha mostra que o efeito do aumento de $\Delta$ é transladar a posição do pico para temperaturas maiores. Os valores abaixo do boxe foram mantidos fixos na simulação, sendo $S=\operatorname{sen} k_{F} R / k_{F} R$, e dá uma medida da distância entre os mínimos de potencial.

Aproveitando a forma da expressão (4.9) encontramos a taxa efetiva de tunelamento $\left(\Delta^{*}\right)$ da seguinte forma 


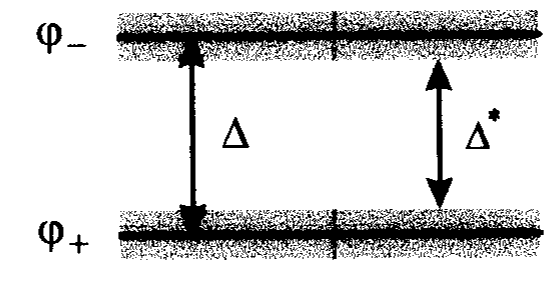

Figura 4.2: Representação esquemática do alargamento das linhas de energia dos estados ligante e antiligante, quando o sistema de dois níveis interage com a BC. Como conseqüência a diferença de mínima energia entre os estados é alterada de $\Delta$ para $\Delta^{*}$.

$$
\frac{k_{B} T_{P}^{*}}{D} \cong 0.417 \Delta^{*}
$$

onde $T_{P}^{*}$ indica a posição do pico, que pode ser determinado através da curva.

Finalmente, a Figura 4.1 mostra o efeito na posição do pico de $C_{i o n}$ devido à $\Delta$. Podemos perceber que o aumento de $\Delta$ faz com que o pico apenas translade para altas temperaturas. Isto é facilmente compreensivel porque quanto maior a diferença entre os estados ligante e antiligante, maior o valor da temperatura a partir da qual o sistema tem os dois níveis acessíveis.

\section{3 - Tunelamento Assistido}

Nas curvas de calor específico da Figura 4.3, $G$ foi mantido constante e verificamos o papel desempenhado por $G_{a}$ no $C_{i o n}$. Podemos perceber que para $G_{a}=0$ e $\Delta \neq 0$, mais precisamente $\Delta=10^{-4} \mathrm{D}$ no caso mostrado, há um pico no $C_{i o n}$ (curva com pontos vermelhos) como já visto anteriormente. Quando retiramos $\Delta$ e colocamos $G_{a} \neq 0\left(G_{a}=5 \cdot 10^{-5} D\right)$ vemos que continua havendo um pico (curva azul), mostrando como esperado, a possibilidade de ocorrer o tunelamento mesmo sem o termo de tunelamento direto. Mostramos também qual deve ser o valor de $G_{a}$ quando $\Delta=0$ para que tenhamos a mesma curva de $C_{i o n}$ do primeiro caso. Encontramos o valor $G_{a}=10^{-4} D$, coincidindo com o valor de $\Delta$, porém, está 
equivalência geralmente não é obedecida quando alteramos, por exemplo, o valor de $G$.

Os efeitos de $G_{a}$ podem, porém, ser muito mais interessantes, como veremos adiante. Antes, contudo, é instrutivo analisarmos o que acontece com os estados de $H_{0}$ quando fazemos uma particular escolha de parâmetros para a qual nosso Hamiltoniano se mapeia no famoso Hamiltoniano de Kondo para uma impureza magnética em metais. Isto é o que faremos na próxima seção, onde mostraremos $C_{i o n}$ para esta situação.

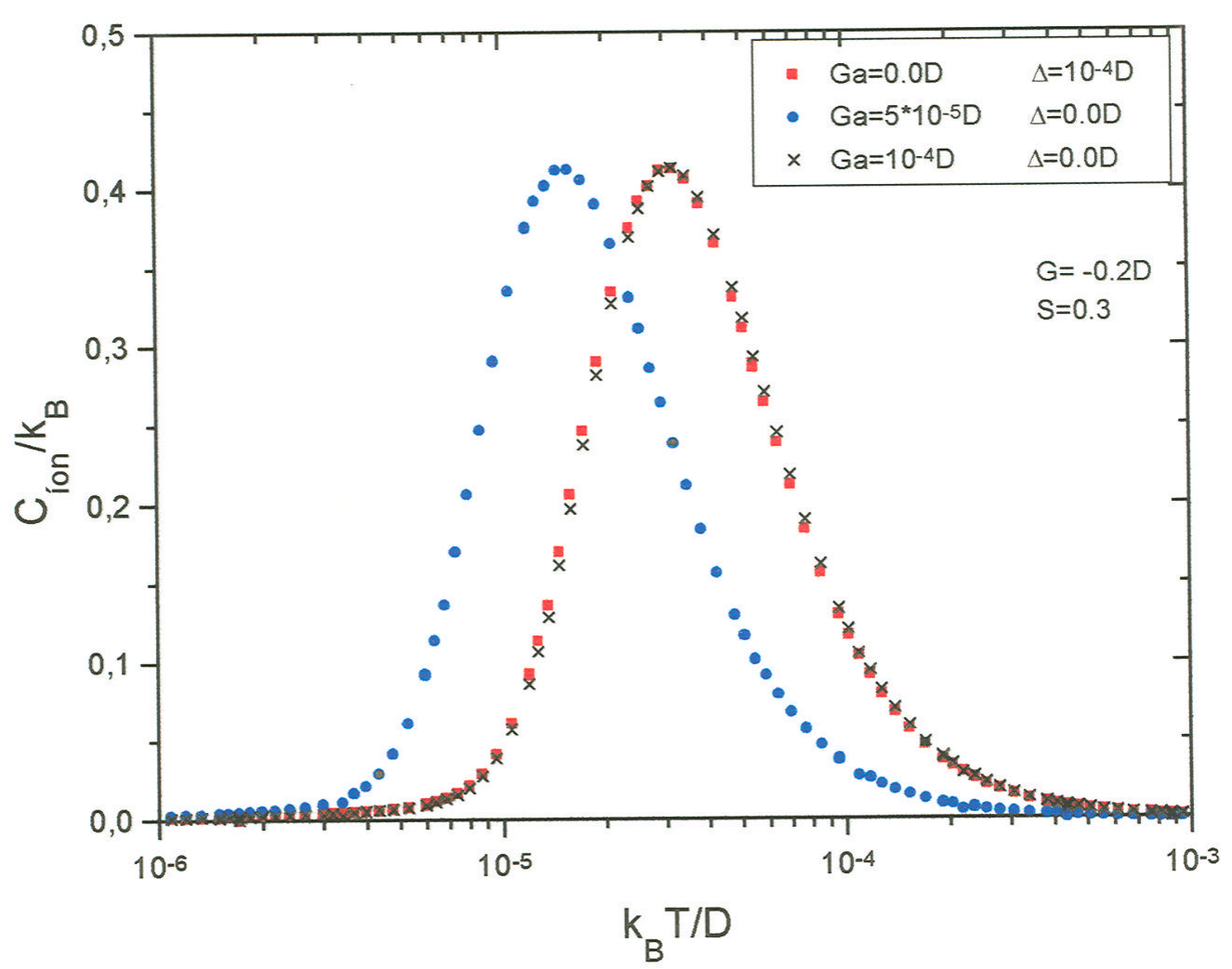

Figura 4.3: As curvas acima mostram que $G_{a}$ pode ter o mesmo efeito de $\Delta$, como pode ser observado pelas curvas azul e $\operatorname{preta}\left(G_{a} \neq 0\right.$ e $\left.\Delta=0\right)$ e pela curva vermelha $\left(G_{a}=0\right.$ e $\left.\Delta \neq 0\right)$. Em particular, mostramos que $G_{a}=10^{-4} \mathrm{D}$ tem exatamente o mesmo efeito que $\Delta=10^{-4} \mathrm{D}$. Contudo esta correspondência numérica é apenas uma coincidência, não acontecendo para qualquer valor de $G$ e $S$. 


\section{4 - O Problema Kondo de Impureza Magnética e o} Tunelamento Assistido.

Mostraremos nesta seção que para uma escolha especial dos parâmetros do nosso Hamiltoniano o mesmo é mapeado no problema Kondo de uma impureza magnética em metal não magnético, conhecido simplesmente como Modelo de Kondo [30]. Para isso, vamos analisar brevemente este problema.

O Modelo de Kondo descreve a interação entre um spin $\vec{\tau}$ e os elétrons de condução de um metal não magnético. Os elétrons da $\mathrm{BC}$ tendem a formar uma blindagem com uma densidade de spin resultante $\vec{s}$ em torno da impureza. A Fig. 4.4 ilustra esse problema.

Figura 4.4 - Representação do modelo de Kondo para uma impureza magética com spin $\vec{\tau}$ em um metal.

O Hamiltoniano de interação para esse problema é dado por

$$
H_{K}=J \vec{s} \cdot \vec{\tau}
$$

onde $J$ é a constante de acoplamento entre impureza e BC. A expressão para a densidade de spin $\vec{s}$ é dada em função das matrizes de Pauli, $\vec{\sigma}$, como segue

$$
\vec{s}=\sum_{k, k^{\prime}, \mu, v} c_{k \mu}^{+} \vec{\sigma}_{\mu \nu} c_{k^{\prime} v},
$$

onde $k$ e $k^{\prime}$ são os momentos dos elétrons da $\mathrm{BC}$, e $\mu$ e $v$ as componentes $\pm 1 / 2$ de spin.

Aplicando a técnica do GRN, mostrada no Capítulo 3, podemos reescrever a Eq. (4.11) como 


$$
H_{k}=2 J s_{z} \tau_{z}+J\left(s_{-} \tau_{+}+s_{+} \tau_{-}\right),
$$

onde

$$
\begin{aligned}
& s_{z}=\left(f_{0+}^{+} f_{0+}-f_{0-}^{+} f_{0-}\right) / 2, \\
& s_{-}=f_{0-}^{+} f_{o+} \mathrm{e} \\
& \mathrm{s}_{+}=f_{0+}^{+} f_{0-},
\end{aligned}
$$

que são operadores que obedecem às relações usuais de comutação de spin.

Voltando ao Hamiltoniano $H_{0}$, Eq. (3.63), vamos analisar o caso em que $A_{+}=A_{-}$, o que implica segundo a Eq. (3.10) que, $k_{F} R=n \pi \operatorname{com}(n=0,1,2, \ldots)$. Vamos ainda considerar que o potencial eletrostático $\bar{G}$ assuma o valor $\bar{G}_{1}$ no termo proporcional a $d_{+}^{+} d_{+}+d_{-}^{+} d_{-}$, e o valor $\bar{G}_{2}$ no termo proporcional a $d_{+}^{+} d_{-}+d_{-}^{+} d_{+}$ Assim,

$$
\begin{aligned}
\Lambda^{1 / 2} H_{0} & =\bar{G}_{1}\left[f_{0+}^{+} f_{0+}+f_{0-}^{+} f_{0-}\right]\left[d_{+}^{+} d_{+}+d_{-}^{+} d_{-}\right]+ \\
& +\bar{G}_{2}\left[f_{0+}^{+} f_{0-}+f_{0-}^{+} f_{0+}\right]\left[d_{-}^{+} d_{+}+d_{+}^{+} d_{-}\right]+ \\
& +\bar{G}_{a}\left[f_{0+}^{+} f_{0+}-f_{0-}^{+} f_{0-}\right]\left[d_{+}^{+} d_{+}-d_{-}^{+} d_{-}\right]+ \\
& +\bar{G}_{a}\left[f_{0-}^{+} f_{0+}-f_{0+}^{+} f_{0-}\right]\left[d_{+}^{+} d_{-}-d_{-}^{+} d_{+}\right]- \\
& -\bar{\Delta} / 2\left(d_{+}^{+} d_{+}-d_{-}^{+} d_{-}\right) .
\end{aligned}
$$

Seguindo, agora, o mesmo tipo de definição das Eqs. (4.14), podemos reescrever algumas partes da Eq. (4.15) através da notação de spin

$$
\begin{aligned}
\Lambda^{1 / 2} H_{0} & =\bar{G}_{1}\left[f_{0+}^{+} f_{0+}+f_{0-}^{+} f_{0-}\right]\left[d_{+}^{+} d_{+}+d_{-}^{+} d_{-}\right]+ \\
& +4 \bar{G}_{a} s_{z} \tau_{z}+\left[\bar{G}_{a}+\bar{G}_{2}\right]\left[s_{+} \tau_{-}+s_{-} \tau_{+}\right]- \\
& -\left[\bar{G}_{a}-\bar{G}_{2}\right]\left[s_{-} \tau_{-}+s_{+} \tau_{+}\right]-\bar{\Delta} \tau_{z},
\end{aligned}
$$


sendo que $\vec{s}$ se refere à pseudospins da $\mathrm{BC}$ e suas componentes são dadas pelas relações (4.14). O termo $\vec{\tau}$ se refere aos pseudospins associados ao ín tunelante $\mathrm{e}$ suas componestes são:

$$
\begin{aligned}
& \tau_{z}=\left(d_{+}^{+} d_{+}-d_{-}^{+} d_{-}\right) / 2, \\
& \tau_{-}=d_{-}^{+} d_{+} \quad \mathrm{e} \\
& \tau_{+}=d_{+}^{+} d_{-}
\end{aligned}
$$

Se fizermos agora a seguinte escolha de parâmetros

$$
\begin{aligned}
& \bar{G}_{a}=\bar{G}_{2} \\
& \bar{G}_{1}=0 \mathrm{e} \\
& \bar{\Delta}=0,
\end{aligned}
$$

obtemos

$$
\Lambda^{1 / 2} H_{0}=4 \bar{G}_{a} s_{z} \tau_{z}+2 \bar{G}_{a}\left(s_{-} \tau_{+}+s_{+} \tau_{-}\right)
$$

que, identificando $2 G_{a}$ com $J$, é o Hamiltoniano de Kondo, Eq. (4.13).

Essa particular escolha de parâmetros nos permite, então, diagonalizar o Hamiltoniano de Kondo como um "subproduto" do nosso Hamiltoniano total.

Definindo, agora, $\vec{S}$ como sendo a soma do pseudospin da $\mathrm{BC}$ com o pseudospin do íon, $\vec{S}=\vec{s}+\vec{\tau}$, verifica-se que $\vec{S}^{2}$ comuta com o Hamiltoniano $H_{N}$, Eq. (3.53), $\left[H_{N}, \vec{S}^{2}\right]=0$, mostrando que o pseudospin total se conserva. A componente $S_{z}$ do pseudospin total também comuta com $H_{N},\left[H_{N}, S_{z}\right]=0$, indicando a conservação desta componente. Estas propriedades de comutação são as mesmas do Modelo de Kondo.

Sabe-se que o modelo de Kondo possui um estado singleto (antiferromagnético) como estado fundamental [33]. Em nosso caso, temos o singleto também como estado fundamental. Para entendermos essa afirmação devemos analisar os auto-estados de $H_{0}$, lembrando que $H_{0}$ é a parte do Hamiltoniano iterativo que carrega todo o acoplamento direto entre íon tunelante e BC. 


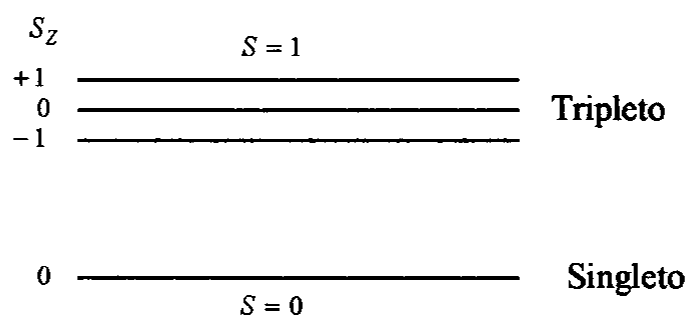

Figura 4.5: Níveis de energia do estado singleto (estado fundamental) e dos estados tripletos. No centro está o valor da soma dos pseudospins associados à $\mathrm{BC}$ e ao íon. À direita, está indicado o valor da componente $S_{z}$. O tripleto foi desenhado com uma certa abertura nos níveis para que tenhamos uma melhor visualização das componentes $S_{z}$.

Antes vamos recordar que para diagonalizar $H_{0}$ utilizamos a base $\left|f_{0+} f_{0-} ; d_{+} d_{-}\right\rangle$. Nessa base os operadores do Hamiltoniano $H_{0}$ atuam da seguinte maneira: $f_{0+(-)}$ destrói um elétron da $\mathrm{BC}$ com paridade par (ímpar), $d_{+(-)}$destrói o ín no estado ligante (antiligante). Os vetores dessa base foram mostrados em (3.64).

Feita a diagonalização e analisado o espectro de energia, verificamos que o estado fundamental é dado por:

$$
\frac{1}{\sqrt{2}}|10 ; 01\rangle-\frac{1}{\sqrt{2}}|0 \quad 1 ; 10\rangle
$$

e é análogo ao estado singleto do modelo de Kondo que possui $S=0$. Dentre os estados excitados, temos o tripleto dado por

$$
\begin{aligned}
& \frac{1}{\sqrt{2}}|10 ; 01\rangle+\frac{1}{\sqrt{2}}|01 ; 10\rangle, \\
& |10 ; 10\rangle \mathrm{e} \\
& |01 ; 001\rangle .
\end{aligned}
$$

Esses estados tripletos possuem $S=1$ e componentes $\mathrm{z}, S_{z}$, iguais a $0,1 \mathrm{e}-1$, respectivamente. A Fig. 4.5 representa esquematicamente os níveis de energia do estado singleto e dos estados tripletos.

A Fig. 4.6 mostra o calor específico neste caso, onde comparamos a curva obtida por nós (curva azul) com a curva universal do calor específico de Kondo [31] 
(curva vermelha). Podemos verificar a ótima concordância entre elas.

Na inserção da Fig. 4.6 mostramos o comportamento linear da curva de calor específico em baixas temperaturas, sendo essa uma característica típica dos sistemas tipo líquido de Fermi, como mostra a expressão do calor específico deste caso [32]

$$
\frac{C}{k_{B}}=\frac{k_{F}}{3 \hbar^{2}} m^{*} k_{B} T
$$

onde $m^{*}$ é a massa efetiva do íon e $k_{F}$ é o momento de Fermi.

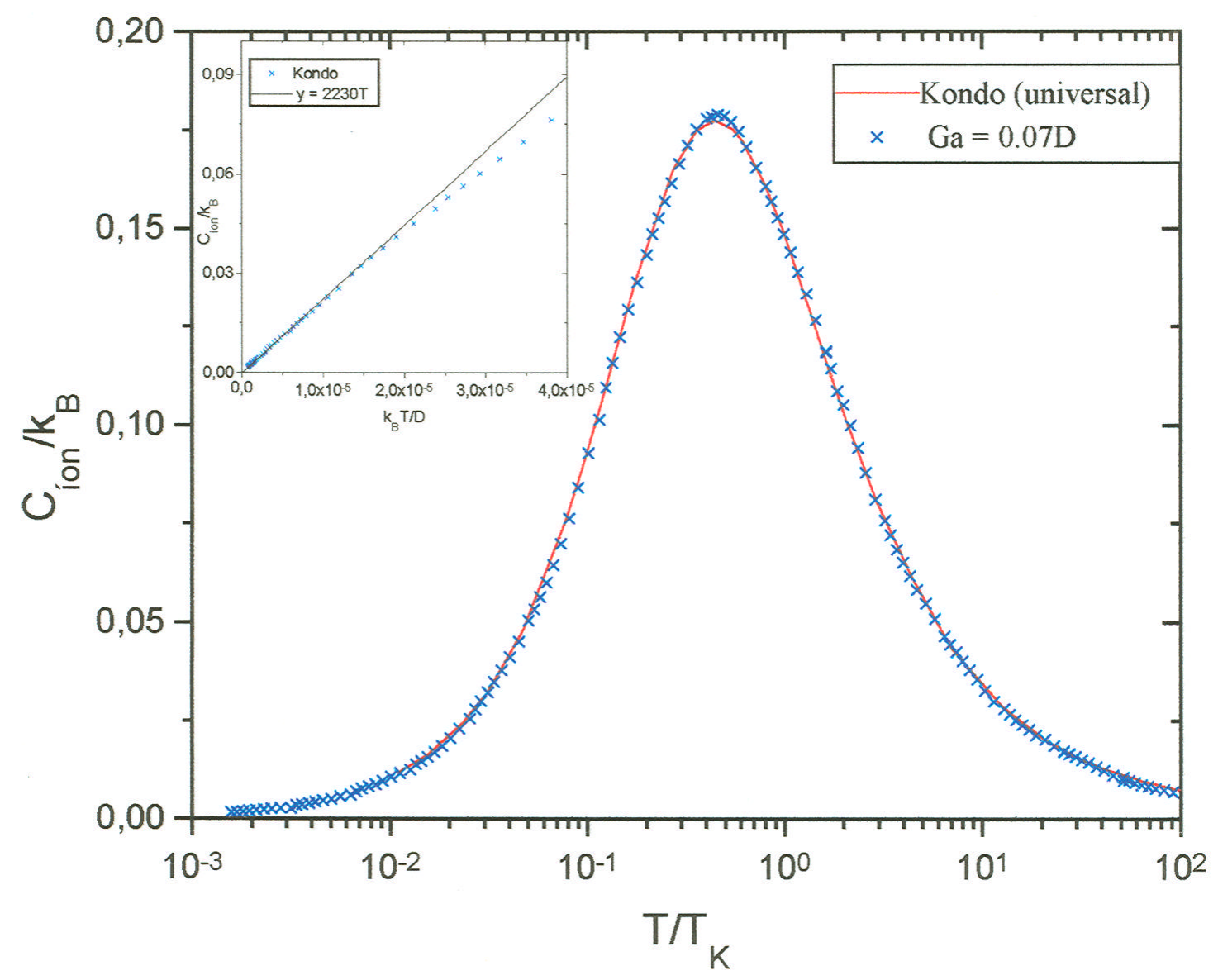

Figura 4.6: A curva vermelha nessa figura representa a curva universal de calor específico para o problema Kondo (os dados foram extraídos da Ref. [31]). A curva azul é o calor específico com nossa escolha de parâmetros. $T_{K}$ no eixo horizontal é a temperatura de Kondo. A inserção mostra o comportamento linear do calor específico em baixas temperaturas, como esperado para um sistema tipo líquido de Fermi.

O $C_{\text {ion }}$ no limite de baixas temperaturas pode ser dado também em termos da temperaturade de Kondo, $T_{K}$. Neste caso [31] 


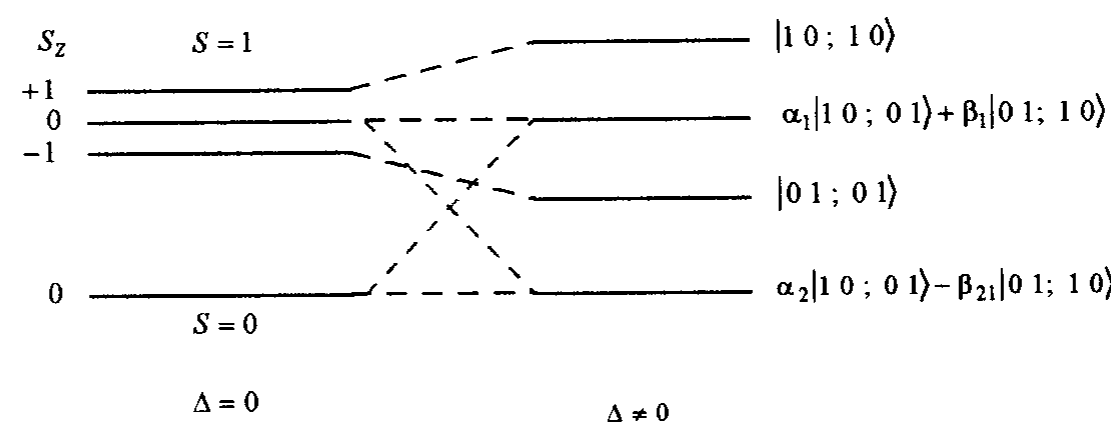

Figura 4.7: Representação esquemática da mistura dos níveis que possuem $S_{z}=0$ quando fazemos $\Delta \neq 0 . \Delta$ funciona como um campo magnético aplicado nos estados do íon, fazendo que os estados que compõe o tripleto se abram, como mostra o lado direito. Mostramos, também, do lado direito os estados de $H_{\Delta}$ que dão origem a cada linha de energia.

$$
\frac{C_{\text {ion }}}{k_{B}}=\frac{\pi}{3 T_{K}} \frac{k_{B} T}{D}
$$

Utilizando o coeficiente angular desta expressão, $\pi / 3 T_{K}$, e o valor do mesmo que aparece na inserção da Fig. 4.6, encontraremos o valor

$$
\frac{k_{B} T_{K}}{D}=0.00047
$$

valor que concorda com o valor de $k_{B} T_{K} / D$ utilizado para normalizar a curva do $C_{\text {ion }}$ (curva azul) da Fig4.6.

Nas próximas seções iremos estudar como se modifica o $C_{\text {ion }}$, a partir da situação Kondo, quando "ligamos" os parâmetros que foram anulados nesta seção.

\section{5 - Situação Kondo incluindo $\Delta \neq 0$}

Quando acrescentamos ao caso Kondo um $\Delta \neq 0$, temos de acordo com a Eq.(4.16)

$$
\Lambda^{1 / 2} H_{0} \equiv H_{\Delta}=4 \bar{G}_{a} s_{z} \tau_{z}+2 \bar{G}_{a}\left[s_{+} \tau_{-}+s_{-} \tau_{+}\right]-\bar{\Delta}\left(\tau_{z}\right)
$$

Podemos interpretar $\Delta$ como um "campo magnético" na direção $\mathrm{z}$ aplicado somente no íon (esta interpretação se justificaria, em um caso magnético, se 
tivéssemos um íon com momento de dipólo magnético grande). Isso faz com que $\vec{S}^{2}$ não comute com o Hamiltoniano $H_{\Delta}$, fazendo com que o pseudospin total não seja mais um bom número quântico. Assim, se olharmos para os estados de $H_{\Delta}$ veremos que os estados tripleto e singleto são destruídos, havendo uma mistura entre os estados que possuem $S_{z}=0$ (Fig.4.7)

$S_{z}$, por sua vez, continua comutando com $H_{N}$, ou seja, continua sendo número quântico.

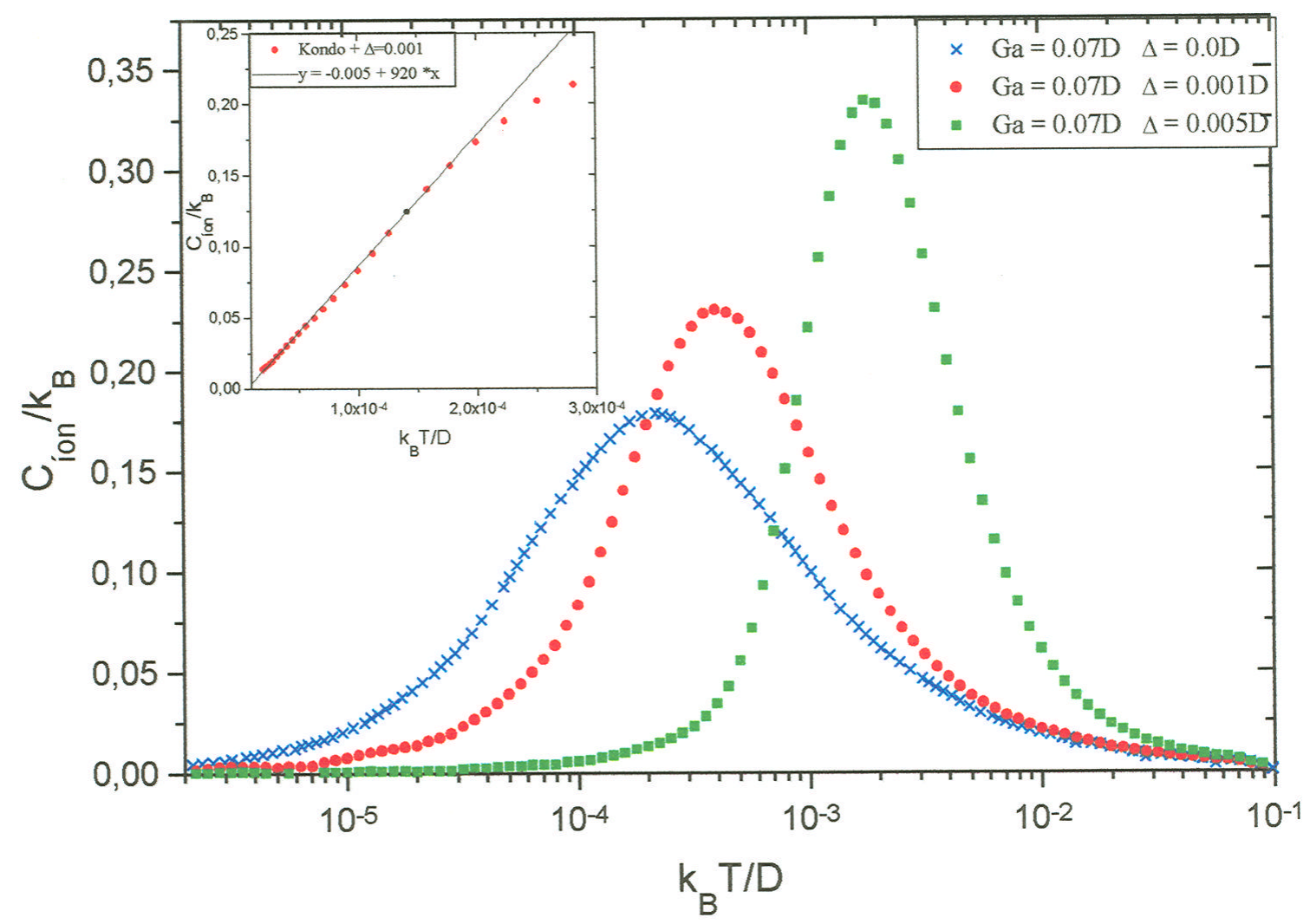

Figura 4.8: Curvas de calor específico; a primeira (curva azul) está no regime Kondo e a segunda (curva vermelha) e terceira (curva verde) foram obtidas acrescentado-se $\Delta \neq 0$. Observe que as duas últimas curvas coincidem em altas temperaturas com o caso Kondo pois, nesta região a energia térmica é muito maior que $\Delta$, ou seja, $\Delta$ é desprezível. A inserção mostra que continua havendo comportamento linear em baixas temperaturas com o acréscimo de $\Delta$.

A Figura 4.8 mostra o calor específico para essa situação e a estamos comparando com o caso Kondo. Percebe-se que agora o pico do calor específico se desloca para temperaturas mais altas, o que significa que os estados do íon não são mais degenerados como no caso Kondo, necessitando uma energia maior para acessar 
o estado excitado e quanto maior o valor de $\Delta$ maior deve ser a energia térmica necessária.

Podemos ver também que para altas temperaturas as curvas coincidem, pois nesta escala de energia a abertura $\Delta$ dos níveis do íon é insignificante, e o sistema se comporta como se esses níveis fossem degenerados, daí reproduzindo a situação Kondo. Com a diminuição da temperatura o sistema começa a perceber a separação dos níveis e isso se reflete no $C_{i o n}$ que passa a não mais coincidir com o caso Kondo.

Percebe-se, também, que quanto maior o valor de $\Delta$ maior o pico do $C_{i o n} \mathrm{e}$, embora não tenhamos mostrado na figura, a tendência é o pico atingir a altura máxima permitida, que é a do sistema de dois níveis mostrado na Fig. 4.1. Isto é devido ao fato de que para grandes valores de $\Delta$ menor é a influência de $G_{a}$ neste caso. Com o aumento do pico do $C_{i o n}$, há um estreitamento na largura dessa curva, já que a área total deve ser igual à $\ln (w)$, onde $w$ é o número de graus de liberdade do sistema, que neste caso vale dois (só temos dois estados acessíveis para o íon).

$\mathrm{Na}$ inserção mostramos que continua havendo comportamento tipo líquido de Fermi em baixas temperaturas. Só que o coeficiente angular da curva é menor do que no caso Kondo, indicando que a massa efetiva do íon tunelante é menor, como mostra a expressão (4.22)

\section{6 - Situação Kondo incluindo $G_{1} \neq 0$.}

Quando consideramos o caso Kondo mais $G_{1} \neq 0$ temos o seguinte Hamiltoniano

$$
\begin{aligned}
& \Lambda^{1 / 2} H_{0} \equiv H_{G 1}=4 \bar{G}_{a} s_{z} \tau_{z}+2 \bar{G}_{a}\left[s_{+} \tau_{-}+s_{-} \tau_{+}\right]+ \\
& +\bar{G}_{1}\left[f_{0+}^{+} f_{0+}+f_{0-}^{+} f_{0-}\right]\left[d_{+}^{+} d_{+}+d_{-}^{+} d_{-}\right] .
\end{aligned}
$$

É fácil perceber que a parte do Hamiltoniano que contém $G_{1}$ é diagonal na base $\left|f_{0_{+}} f_{0_{-}} ; d_{+} d_{-}\right\rangle$. Nessa situação o Hamiltoniano total comuta com $S_{z}$ e com $\vec{S}^{2}$, preservando os estados singleto e tripleto. O parâmetro $G_{1}$ é um potencial 
eletrostático que atua no íon tunelante. Na situação em questão, interpretamos $H_{0}$ como sendo o Hamiltoniano de Kondo para uma partícula carregada e com spin.

A Fig. 4.9 mostra o $C_{i o n}$ para esta situação e também para o caso Kondo. Percebe-se que o pico desloca-se para temperaturas baixas ao fazermos $G_{1} \neq 0$ (as curvas são invariantes pela troca de sinal de $G_{1}$ ). Essa é uma situação equivalente àquela da seção 4.2 , onde vimos que o efeito provocado pelo aumento do valor de $G$ é justamente deslocar o pico de $C_{i o n}$ para temperaturas baixas. Como $G_{1}$ é originário de $G$, ele possui o mesmo efeito.

$\mathrm{Na}$ inserção vemos, novamente, o comportamento linear do $C_{i o n}$, mas o coeficiente angular é maior que o caso Kondo indicando um massa efetiva do íon maior.

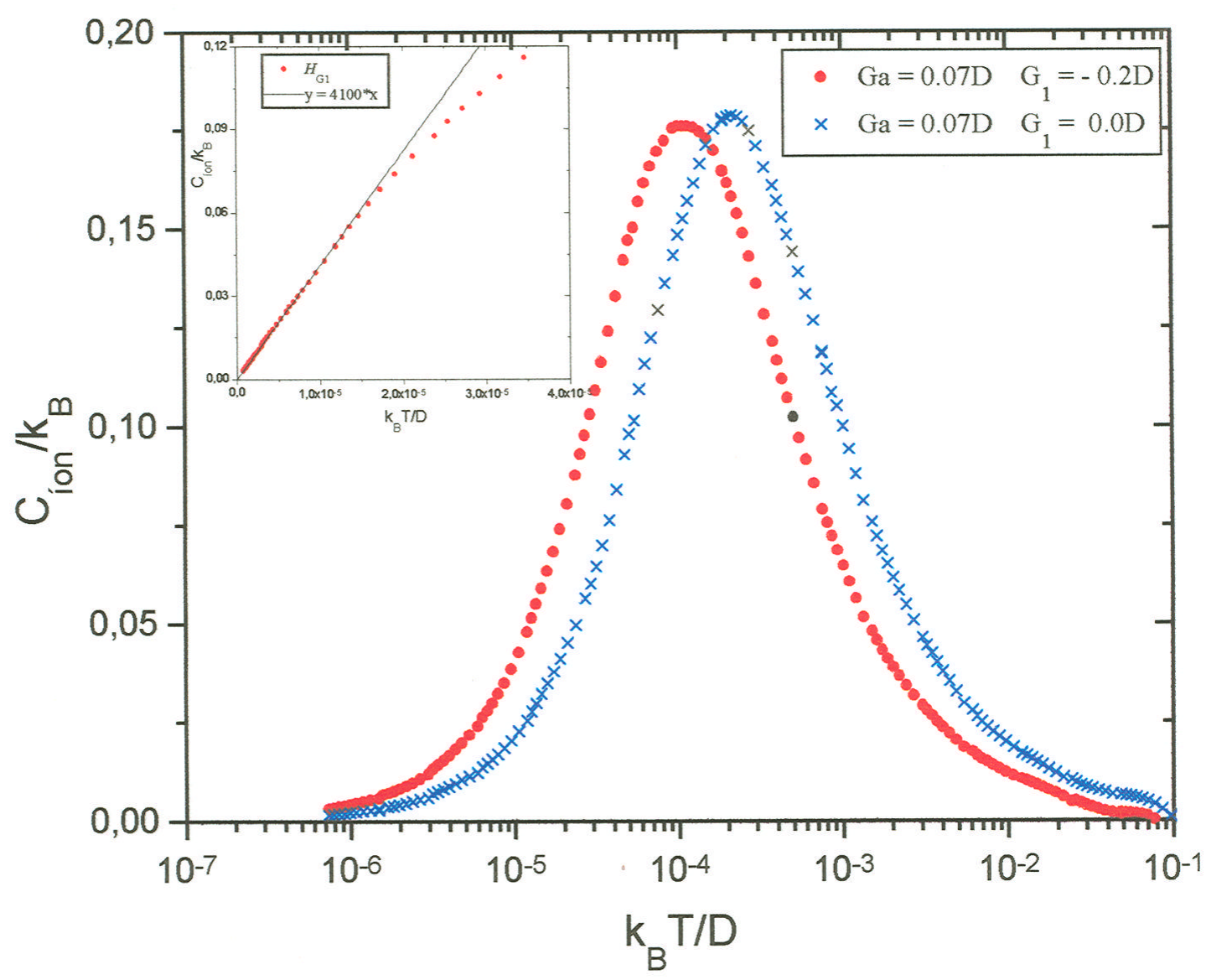

Figura 4.9: Curvas de calor específico: uma no regime Kondo (curva azul) e a outra com $G_{1} \neq 0$ (curva vermelha). A inserção mostra o comportamento linear em baixas temperaturas da curva deste segundo caso. 


\section{7 - O Comportamento Geral do Calor Específico com $G_{a}$}

Nas seções anteriores mostramos o comportamento do calor específico para vários casos, tendo como ponto de partida a situação Kondo. A intenção era obtermos uma "intuição" fisica do problema a partir da análise dos níveis de energia e auto-estados de $H_{0}$. Essa análise, contudo, se torna muito dificil quando vamos para os próximos Hamiltonianos da seqüência da Eq. (3.53). Quando consideramos a presença de todos os parâmetros, ou seja, quando estamos bem distante da situação Kondo, essa análise se complica ainda mais. Porém, o comportamento de $C_{i o n}$ nessa situação se torna muito interesante, como pode ser observado na Figura 4.10. Nela estamos mostrando várias curvas de calor específico para as quais o parâmetro $G_{a}$ foi gradativamente aumentado a partir de $G_{a}=0$ (pontos pretos). Nesta Figura estamos considerando o caso de um íon negativamente carregado, $G>0$.

Pode-se observar, agora, o comportamento geral do efeito do tunelamento assistido nas curvas de calor específico. Para valores de $G_{a}$ que vão até aproximadamente $G_{a}=4 \cdot 10^{-4} \mathrm{D}$ (curvas vermelhas) o pico do calor específico se desloca para baixas temperaturas, mostrando que $\Delta^{*}$ está diminuindo. Ao continuarmos aumentando o valor de $G_{a}$ o pico se desloca para temperaturas mais altas, indicando, agora, que $\Delta^{*}$ começa a aumentar (as curvas azuis mostram esse comportamento). Note que para $G_{a}=5 \cdot 10^{-3} \mathrm{D}, \Delta^{*}$ é maior que $\Delta$, sendo que isso nunca ocorre quando estudamos $\Delta^{*}$ sem a presença de $G_{a}$, ou seja, $\Delta^{*}<\Delta$ para $G_{a}=0$.

O deslocamento do pico para baixas temperaturas e depois para altas com o aumento de $G_{a}$, sugere que há momentos em que o efeito de $G_{a}$ está se somando preferencialmente ao efeito de $G$ (lembre-se que o aumento de $G$ desloca o pico da curva para baixas temperaturas), e em outros momentos, $G_{a}$ se soma com mais intensidade ao efeito de $\Delta$ (aumentar $\Delta$ desloca o pico para altas temperaturas).

Quando estamos próximo do ponto de retorno, $G_{a}=4 \cdot 10^{-4} D$ na Fig. 4.10, surgem oscilações mais dificeis de serem eliminadas. Neste caso, utilizamos o 
procedimento intercalado com quatro valores de $z$. Assim, o calor específico fica suavizado fazendo [26]

$$
C_{i o n}(T)=\frac{C_{i o n}(T, 0.125)+C_{i o n}(T, 0.375)+C_{i o n}(T, 0.625)+C_{i o n}(T, 0.875)}{2}
$$

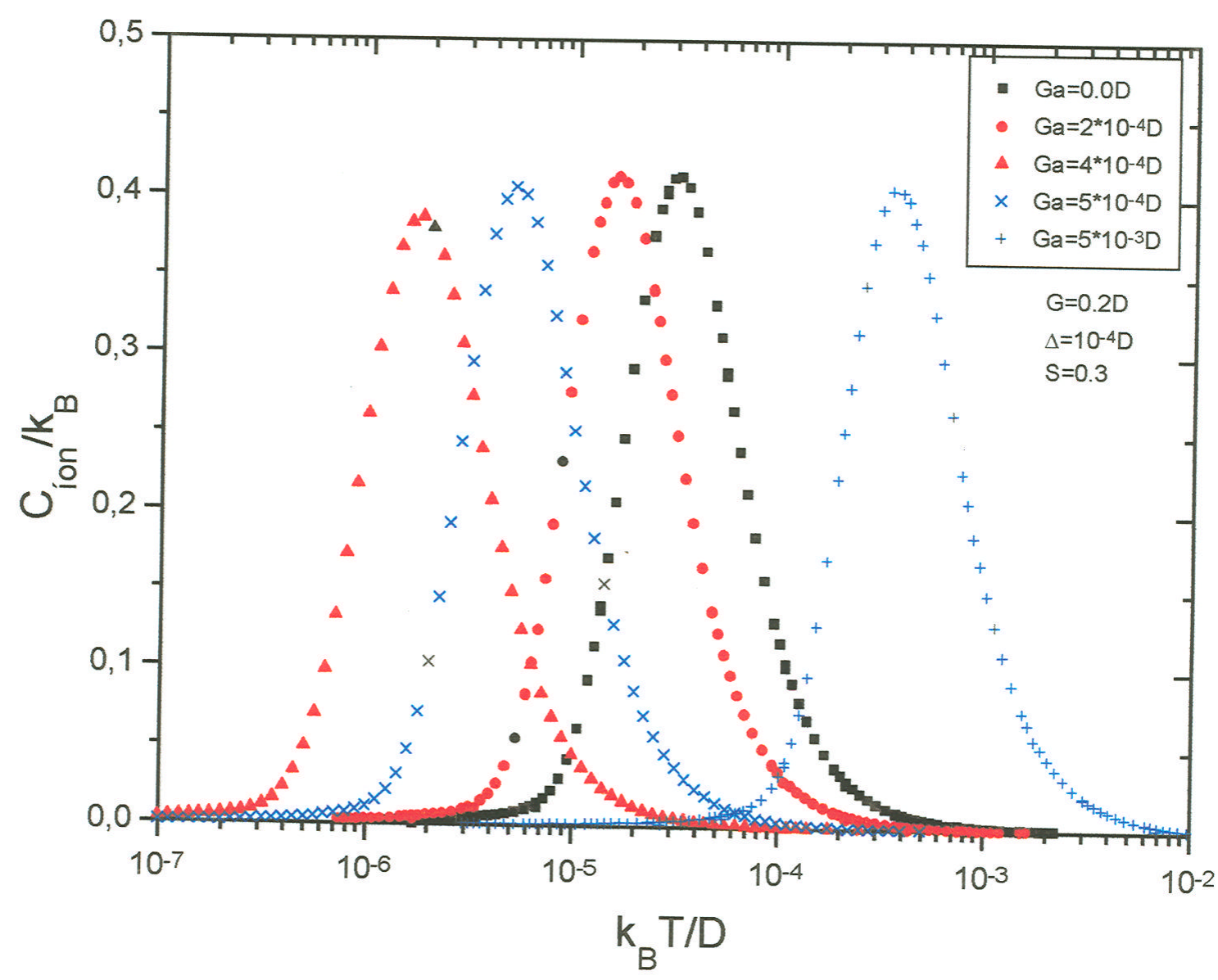

Figura 4.10: Várias curvas de calor específico, mostrando o comportamento geral do efeito do tunelamento assistido. Observa-se que o pico se desloca para baixas temperaturas com o aumento de $G_{a}$ até um certo valor, e então, ele começa a se deslocar para altas temperaturas.

O mesmo comportamento da Fig. 4.10 é encontrado quando $G<0$ e $G_{a}>0$. Contudo, quando trabalhamos com valores de $G_{a}$ menores que zero a curva de $C_{\text {ion }}$ sempre se desloca para temperaturas mais altas quando o módulo do valor de $G_{a}$ é aumentado. Ou seja, $G_{a}<0$ sempre se comporta semelhante ao termo $\Delta$.

Este comportamento das curvas de $C_{\text {ion }}$ nos leva a estudar a taxa de tunelamento efetivo com mais detalhes. Na próxima seção faremos isso. 


\section{8 - Cálculo da Taxa Efetiva de Tunelamento}

Kondo definiu a taxa efetiva de tunelamento, quando não temos tunelamento assistido, da seguinte forma [1]:

$$
\Delta^{*}=2[\varepsilon(0)-\varepsilon(\Delta)]
$$

onde $\varepsilon(\Delta)$ é a energia do estado fundamental do sistema (quando $\Delta=0$ não temos tunelamento).

Utilizando teoria de perturbação, em baixas temperaturas, Kondo desenvolveu a Eq. (4.28) até chegar à expressão,

$$
\frac{\Delta^{*}}{\Delta} \sim\left(\frac{\Delta}{D}\right)^{\eta} \quad \text { para } \quad k_{B} T<<D
$$

Kondo determinou o expoente $\eta$ de maneira aproximada. Posteriormente, Yamada el. al. [3] encontraram a expressão exata para esse parâmetro:

$$
\eta=\frac{K}{K-1} \quad \text { com } \quad K=\frac{1}{\pi^{2}} \operatorname{arcsen}^{2}\left(\sqrt{1-S^{2}} \operatorname{sen} \delta\right)
$$

onde $S$ é dado pela Eq. (4.7) e $\delta$ é a defasagem na função de onda dos elétrons de condução no nível de Fermi provocado pelo potencial Coulombiano e é dado pela Eq.(3.72).

Como o modelo que estudamos possui, além de $\Delta$, o termo de tunelamento assistido, $G_{a}$, fizemos uma redefinição da taxa efetiva:

$$
\Delta^{*}=2\left[\varepsilon(0,0)-\varepsilon\left(\Delta, G_{a}\right)\right]
$$

sendo $\varepsilon\left(\Delta, G_{a}\right)$ a energia do estado fundamental. Indicaremos $\Delta^{*}$ da Eq. (4.28) por $\Delta^{\sim}$. Com as energias obtidas da diagonalização feita pelo GRN, calculamos $\Delta^{*}$ a partir desta equação. Os resultados podem ser observados na Fig. 4.11 onde fizemos 
o gráfico da razão $\Delta^{*} / \Delta$ em função de $G_{a}$. Cada uma destas curvas foi obtida para um valor de $G$ diferente. Podemos observar, agora, com mais clareza o comportamento de $\Delta^{*}$ que tínhamos verificado no estudo do calor específico. Em particular, o ponto de retorno das curvas de calor específico corresponde ao mínimo de $\Delta^{*} / \Delta$. Podemos ver que $\Delta^{*}$ é maior que $\Delta$ quando o módulo da taxa de tunelamento assistido é grande, mostrando que o íon tunela com uma freqüênica maior que da partícula livre. À medida que diminuímos o valor $G_{a}$ em módulo, a razão $\Delta^{*} / \Delta$ diminui linearmente até atingir um ponto de mínimo. Verifica-se numericamente que a taxa efetiva de tunelamento nesta região de mínimo chega a ser duas ordem de grandeza menor que a taxa livre, tendendo a localizar a partícula. Denominaremos o valor $G_{a}$ onde ocorre o mínimo de $\Delta^{*}$ por $G_{a_{\min }}$.

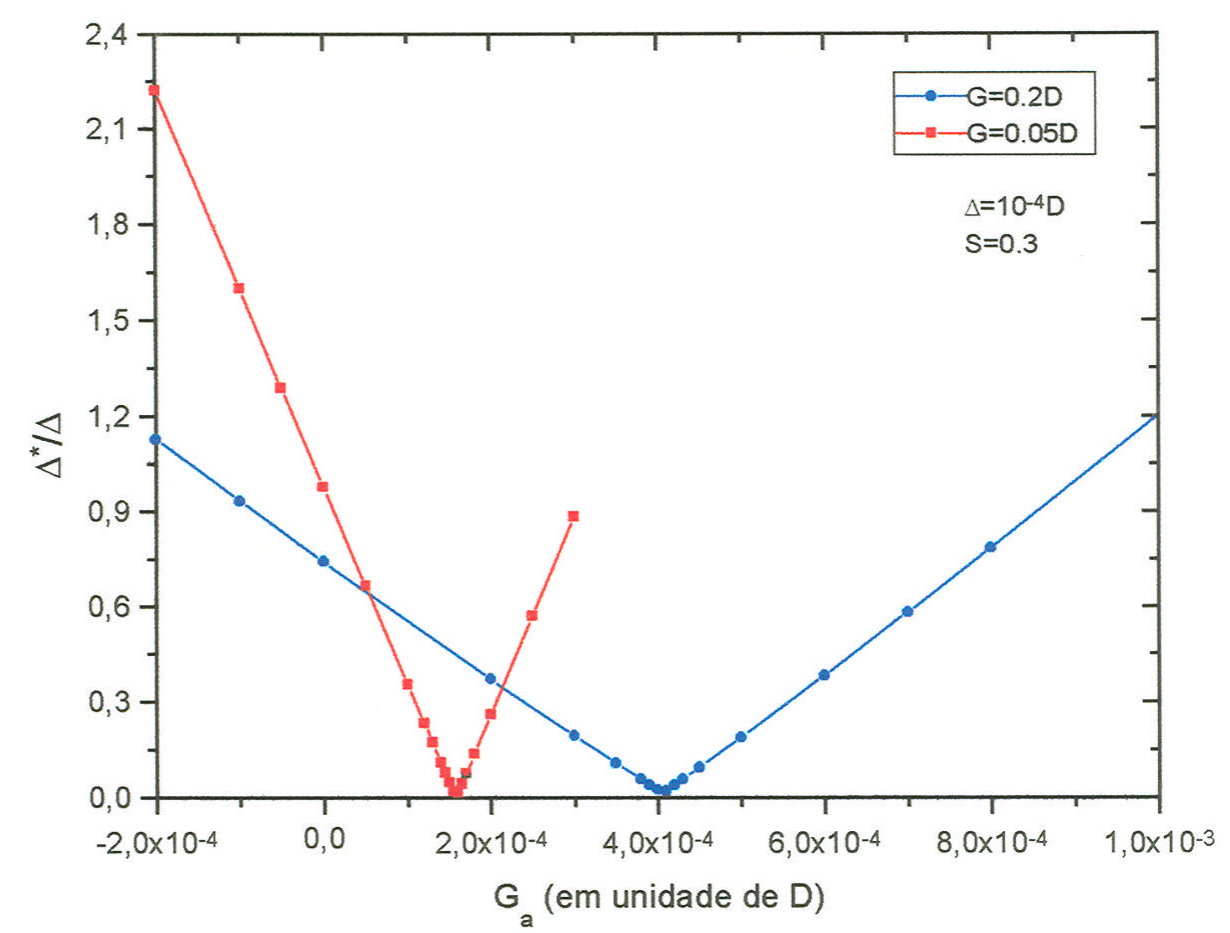

Figura 4.11: As curvas acima mostram o comportamento de $\Delta^{*}$ em função do parâmetro de tunelamento assistido, $G_{a}$. A curva azul foi obtida para $G=0.2 D$ e a curva vermelha para $G=0.05 D$. Observe que existe um valor de $G_{a}$ em cada caso que torna a taxa efetiva de tunelamento mínima. Denominamos esse valor de $G_{a_{\min }}$ e ele é menor quanto menor o valor de $G$. 


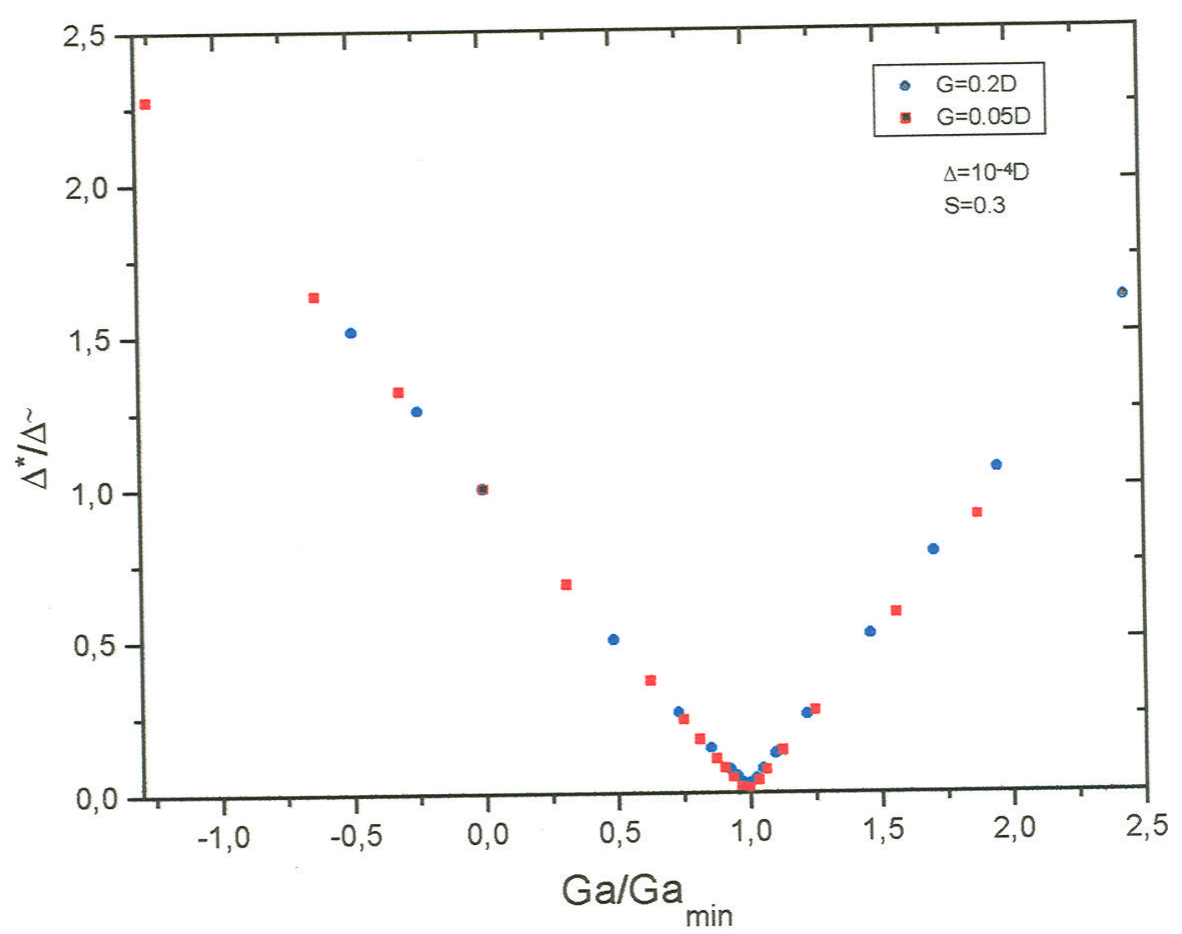

Figura 4.12: As curvas acima mostram que o comportamento das curvas da Fig. 4.11 coincidem quando normalizamos os valores de $G_{a}$ pelo seu respectivo $G_{a_{\min }}$ e os valores de $\Delta^{*}$ por $\Delta^{\sim}$, sendo esta a taxa efetiva de tunelamento quando $G_{a}=0$.

A Fig. 4.11 mostra, também, que quanto menor o valor de $G$, menor é o valor de $G_{a_{\min }}$. Para os dados usados na figura encontramos os seguintes valores aproximados para $G_{a_{\min }}$

$$
G_{a_{\min }} \approx 4.1 \cdot 10^{-4} D \quad \text { para } \quad G=0.2 D
$$

e

$$
G_{a_{\min }} \approx 1.6 \cdot 10^{-4} D \quad \text { para } \quad G=0.05 D
$$

O comportamento linear das curvas não é observado com tanta precisão quando $G_{a}$ é muito grande ( $G_{a}=0.1 D$, por exemplo), porém esses valores não são fisicamente aceitáveis (as curvas de $C_{i o n}$, por exemplo vão estar em regiões de temperatura muito alta, equivalente a $5 \mathrm{eV}$ ). 
O formato das curvas da Fig. 4.11 sugere que se normalizarmos os valores de $G_{a}$ de cada curva pelo seu respectivo $G_{a_{\min }}$ e, ainda, se dividirmos os valores de $\Delta^{*}$ por $\Delta^{\sim}$ (neste caso vale a expressão (4.29)), teremos as curvas coincidindo. A Fig. 4.12 mostra este resultado.

Os resultados mostrados nas Figs. 4.11 e 4.12 foram obtidos para $\Delta=10^{-4} D$. Quando aumentamos este valor em uma ordem de grandeza, o valor de $G_{a_{\min }}$ aumenta aproximadamente na mesma proporção, porém $\Delta^{\sim}$ é apenas uma ordem de grandeza menor que a taxa livre. E ainda, a linearidade mostrada na Fig. 4.11 fica restrita a uma região bem menor de valores de $G_{a}$. Esse ponto, no entanto, merece ainda mais atenção. 


\section{Capítulo 5}

\section{Conclusões e Sugestões}

Neste trabalho calculamos o calor específico de um sistema formado por um íon sem spin que tunela entre dois mínimos de potencial de um metal amorfo e que interage eletrostaticamente, através de uma constante de acoplamento $G$, com os elétrons de condução do metal. Dois processos de tunelamento distintos foram considerados: o primeiro é o tunelamento intrínseco representado pela taxa de tunelamento $\Delta$; o segundo é o tunelamento via espalhamento dos elétrons de condução ou simplesmente tunelamento assistido, cuja intensidade é indicada por $G_{a}$, sendo que propomos uma forma específica para esse tunelamento. Utilizamos o Modelo de Kondo de Tunelamento para representar esse sistema acrescido do termo representativo do tunelamento assistido. No Capítulo 2 mostramos que esse Hamiltoniano respeita a forma mostrada por Zawadowski para interação entre um sistema de dois níveis e um banho fermiônico.

Seguindo os passos de trabalhos precedentes, utilizamos a técnica do Grupo de Renormalização Numérico para diagonalizar o Hamiltoniano. Essa técnica se baseia na discretização logarítmica da banda de condução do metal, de modo a se enfatizar as regiões próximas ao nível de Fermi, região de interesse no estudo de propriedades termodinâmicas em baixas temperaturas. Após aplicada a técnica, mostramos que para uma escolha adequada de parâmetros o Hamiltoniano de Kondo de Tunelamento é mapeado no Hamiltoniano de Kondo de uma impureza magnética em metal. Pudemos, neste caso, usar a linguagem de spin para tratar o problema.

Mostramos no Capítulo 4, curvas de calor específico para algumas combinções de $G, \Delta \mathrm{e} G_{a}$ diferentes. Verificamos, primeiramente, o efeito dos parâmetros $G$ e $\Delta$, sem a presença de tunelamento assistido, a partir da curva de calor específico de um sistema de dois níveis simples. verificamos que o aumento no 
valor de $\Delta$ simplesmente translada a curva no eixo das temperaturas. $O$ parâmetro $G$ renormaliza a taxa de tunelamento, fazendo que a curva se desloque para temperaturas mais baixas e diminua o seu valor de pico.

Ao adicionarmos o parâmetro $G_{a}$, mostramos que as curvas de calor específico se deslocam para baixas temperaturas até atingir um valor mínimo quando esse parâmetro é aumentado. Ao continuarmos aumentando $G_{a}$, as curvas se deslocam novamente para temperauras altas. Esse comportamento nos levou a estudar a taxa efetiva de tunelamento, $\Delta^{*}$, em mais detalhes. Para isso, ampliamos a definição de Kondo para essa taxa de modo a levar em conta, também, os efeitos do tunelamento assistido. Pudemos observar que $\Delta^{*}$ varia bastante quando alteramos o valor de $G_{a}$, sendo que $\Delta^{*}$ pode ser maior que $\Delta$ para certos valores de $G_{a}$ indicando que a frequência de tunelamento é aumentada. Ou então, pode ser muito menor que $\Delta$, indicando que a partícula está tendendo a se localizar. Isto sugere que $G_{a}$ se soma preferencialmente ao efeito de $G$ até um certo valor crítico de $G_{a}$, e então, passa a se somar com mais intensidade ao efeito de $\Delta$. O valor crítico de $G_{a}$ depende dos valores de $G, \Delta$ e $S=\operatorname{sen}\left(k_{F} R\right) / k_{F} R$ utilizados.

Finalmente, mostramos que é possivel fazer com que diversas famílias de curvas de $\Delta^{*} / \Delta$ contra $G_{a}$ coincidam quando normalizamos o conjunto de $G_{a}$ pelo seu respectivo $G_{a}$ crítico e também fazemos a divisão do conjunto de $\Delta^{*}$ por $\Delta^{\sim}$, sendo esta a taxa efetiva de tunelamento quando $G_{a}=0$.

Como sugestões para futuros trabalhos propomos um estudo mais amplo do comportamento de $\Delta^{*} / \Delta^{\sim}$ em função de $G_{a} / G_{a_{\min }}$, para diversos valores de $G, \Delta$ e $S$, para verificar a extensão da lei de escala sugerida pela Fig. 4.12. O próprio ponto de retorno das curvas de calor específico, Fig. 4.10, merece uma explicação fisica mais profunda que a dada nesta dissertação. $\mathrm{O}$ cálculo da densidade espectral desse modelo poderia ser uma sugestão valiosa nessa direção.

Por fim, a aproximação feita no Capítulo 3, Eqs. (3.32), que se resume em ser $k=k_{F}$, já pode ser removida [24], e fica como sugestão para futuros trabalhos. Cálculos preliminares mostram que no caso $G_{a}=0$ a aproximação $k=k_{F}$ não muda as curvas de calor específico. Com o termo assistido ainda não temos indicação. 


\section{Referências}

[1] J. Kondo, Physica 84B, 40 (1976).

[2] A. O. Caldeira e A. J. Legget, Physical Review Letters 46, 211 (1981).

[3] K. Yamada, A. Sakurai e M. Takeshige, Progress of Theoretical Physics 70, 73 (1983).

[4] A. Zawadowski et. al., Physical Review B 56, 12947 (1997).

[5] W. A. Philips, J. Low. Temp. Phys., 7, 161 (1971); P W. Anderson, B. I. Halperin e C. M. Varma, Philos. Mag. 25, 1 (1972) .

[6] R. W. Cochrane et. al. , Physical Review Letters 35, 676 (1975); G. Zaránd e K. Vladár, Orbital Kondo-Effect from Tunneling Impurities, a publicar.

[7] K. Vladár e A. Zawadowski, Physical Review B 28, 1564, 1582, 1596 (1983).

[8] G. Zaránd, Solid State Comm. 86, 413 (1993).

[9] G. Zaránd e A. Zawadowski, Physical Review Letter 72, 542 (1994).

[10] G. Zaránd e A. Zawadowski, Physical Review B 50, 932 (1996).

[11] A. Zawadowski, Physical Review Letter 45, 211 (1980).

[12] G. Zaránd, Physical Review Letter 77, 3609 (1996).

[13] J. Kondo, Physica 84B, 207 (1976).

[14] K. G. Wilson, Reviews of Modern Physics 47, 773 (1975).

[15] H. R. krishina-Murthy, J. W. Wilkins e K. G. Wilson, Physical Review B 21, 1003 (1980).

[16] M. Yoshida, M. A. Whitaker, L. N. Oliveira, Physical Review B 41, 9403 (1990).

[17] João Vítor B. Ferreira, Dissertação de Mestrado, Instituto de Física de São Carlos (1995).

[18] V. L. Líbero, L. N. Oliveira, Physical Review Letter 65, 2042 (1990).

[19] Mahan, Many Particle Physics, Plenum Press, New York, pag. 19 (1981).

[20] L. N. Oliveira, V. L. Líbero, H. O. Frota e M. Yoshida, Physica B 171, 61 (1991). 
[21] Silvia Martins dos Santos, Dissertação de Mestrado, Instituto de Física de São Carlos (1997).

[22] B. A. Jones, C. M. Varma, J. W. Wilkins, Physical Review Letter 61, 125, (1988).

[23] A. J. Millis, B. G. Ketliar, B. A. Jones, Field Theories in Condensed Matter Physics, addison-Wesley, Redwood City,CA (1990).

[24] W. L. C. Lima, Tese de Doutorado, Instituto de Física de São Carlos (1997).

[25] W. C. Oliveira, Tese de Doutorado, Instituto de Física de São Carlos (1994).

[26] S. C. Costa, C. A. Paula, V. L. Líbero e L. N. Oliveira, Physical Review B 55, 30 (1997)

[27] J. L. N. Mello, Tese de Doutorado, Instituto de Física de São Carlos (1992).

[28] H. B. Pang e D. L. Cox, Physical Review B 44, 9454 (1991).

[29] H. R. krishina-Murthy, J. W. Wilkins e K. G. Wilson, Physical Review B 21, 1044 (1980).

[30] J. Kondo, Progress of Theoretical Physics 32 (01), 37 (1964).

[31] H. U. Desgranges, K. D. Schotte, Physics Letters 91A, 240 (1982).

[32] N. W. Ashcroft e N. D. Mermin, Solid State Physics, (1976).

[33] P. Noziéres, Le Journal de Physique 39, 1117 (1978). 